\title{
AAL in Anwendungsszenarien
}

\section{Till Hänisch}

Die Beiträge dieses Kapitels wurden von Studierenden der DHBW Heidenheim im Rahmen eines Integrationsseminars geschrieben (Anm.: bis auf den Artikel zum Thema Digitale Phänotypisierung, der im Rahmen einer ähnlichen Veranstaltung an der Hochschule Aalen entstanden ist). Das Integrationsseminar, eine Besonderheit der Dualen Hochschule, hat diesen Namen, weil es - auf mehreren Ebenen - zusammenbringt, was sonst leider oft mehr oder weniger getrennt bleibt.

Es bringt zum einen Themen aus der Forschung und die praktischen Erfahrungen der Studierenden zusammen, die so lernen (sollen), wie der Transfer von akademischer Erkenntnis in die Praxis der Unternehmen oder Einrichtungen stattfinden kann. Es bringt weiterhin Studierende mit Betreuer*innen aus der Praxis und der akademischen Welt dazu, zusammen an einem anspruchsvollen Thema zu arbeiten, das beiden Parteien neue Perspektiven bietet. Und es bringt in diesem speziellen Fall Studierende und Dozent*innen aus verschiedenen Fakultäten, der (Wirtschafts-) Informatik und der Angewandten Gesundheits- und Pflegewissenschaften zusammen und versucht sich damit am interdisziplinären Austausch. Heraus aus der „Blase“ des eigenen Studienthemas, andere Sichten und Themen aufgreifen und interdisziplinär arbeiten.

Dabei ist es auch diesmal gelungen, interne und externe Autor*innen mit langjähriger Berufs- und wissenschaftlicher Erfahrung mit jungen Menschen im Studium zusammen zu bringen, um gemeinsam ein Buch zu schreiben. Was anfangs ein Experiment war, hat sich bewährt. Dies ist bereits das vierte Werk in dieser Reihe.

T. Hänisch $(\varangle)$

DHBW Heidenheim, Heidenheim, Deutschland

E-Mail: till.haenisch@dhbw-heidenheim.de 
Bisher wurden in diesen Seminaren in Heidenheim Themen wie das Internet der Dinge, Industrie 4.0 oder Blockchains berücksichtigt. Dies sind Themen, die sich aufgrund ihrer Aktualität (noch) nicht im Curriculum finden, die aber in der Praxis relevant sind. Dieses Mal ging es um das Thema Ambient/Active Assisted Living (AAL). Das ist zwar nicht neu, aber gesellschaftlich wichtig. Insbesondere, weil in diesem Bereich der Transfer von der akademischen Forschung in die breite Nutzung bisher nicht gelungen ist.

Die konkreten Themenstellungen der einzelnen Artikel stammen von den Studierenden selbst und wurden nicht von den Betreuer*innen vorgegeben. Wir haben uns entschlossen, die Beiträge genauso zu belassen, wie sie von den Studierenden am Ende des Seminars abgegeben wurden. Um Kontext zu schaffen und Brücken zu bauen haben die jeweiligen Betreuer*innen den Artikeln ein kurzes Vorwort zur Seite gestellt.

\subsection{Auswirkungen eines humanoiden Roboters auf das Wohlbefinden von hospitalisierten Kindern in der Pädiatrie}

Vorwort der Betreuer*innen: Pia Beyer-Wunsch, Christopher Reichstein

Humanoide Roboter halten Einzug in das alltägliche Leben, und können auf vielfältige Weise eingesetzt werden. Gerade durch ihre menschlichen Züge in Form von Gestik und auch begrenzter Mimik sind sie für kommunikative und soziale Interaktion prädestiniert. Die Folge der Mensch-Maschine-Interaktion können intensive emotionale Reaktionen der Nutzer sein.

Genau an diesem Punkt setzt der Versuch an, eine Anwendung zu entwickeln, um bei hospitalisierten Kindern, welche sich häufig in sehr anstrengenden und nicht selten psychisch belastenden Klinikaufenthalten befinden, positive Emotionen anzuregen. Interventionen, ein anderer Tagesrythmus oder auch Schmerzen prägen nicht selten die Zeit in der Klinik. Die entwickelte Anwendung sollte den Kindern dazu verhelfen negative Emotionen in positive umzuwandeln, und ein Wohlgefühl zu erzeugen. Ziel der Untersuchung ist folglich die Verifikation positiver Effekte auf die Kinder, insbesondere während der Intervention einer Pflegekraft.

Für die Untersuchung wurde ein Experiment mit dem humanoiden Roboter „Pepper“ auf der Kinderstation am Universitätsklinikum Ulm durchgeführt. Dies geschah kurz vor dem Ausbruch der Pandemie im März 2020.

Simon Bäuerle, Matthias Eck, Nadine Mayer, Nico Rau, Dennis Sakacilar, Sarah Schwer und Nadine Zeh. 


\section{Hintergrund}

Aktuelle Studien beschäftigen sich zunehmend mit den Möglichkeiten neuer technologischer Errungenschaften in der Pflege, insbesondere von älteren und hilfebedürftigen Menschen, und verweisen immer wieder auf bestehende Herausforderungen, wie dem Fachkräftemangel, dem Kosten- und Zeitdruck sowie dem demographischen Wandel (Boznan, 2019; Mayer et al., 2018; Zhang et al., 2018). Dabei können neue Ansätze der Digitalisierung, der künstlichen Intelligenz und auch der Robotik hilfebedürftige Menschen, aber auch das Pflegepersonal unterstützen (Archibald \& Barnard, 2018; Kumar 2018). Während vergangene Untersuchungen vermehrt auf die Potenziale neuer Digitalisierungsansätze in der ambulanten und stationären Pflege älterer Menschen abzielen, beschäftigt sich das vorliegende Experiment mit den Chancen des Einsatzes von Robotern in der stationären Pflege von Kindern.

Hintergrund ist, dass Fachkräfte in einem Krankenhaus, nicht nur in der Pflege von Erwachsenen, sondern auch von Kindern, täglich eine Vielzahl von routinemäßigen Aufgaben, wie z. B. das Messen des Blutdrucks und der Körpertemperatur oder das Wechseln von Verbänden, zu erledigen haben. Ein wesentlicher Unterschied zwischen der Pflege von Erwachsenen und Kindern liegt hierbei darin, dass Pflegefachkräfte oft mit unterschiedlichen Empfindlichkeiten und Reaktionen während der Pflegemaßnahmen von Kindern konfrontiert werden. Dass Kinder diese Routineaufgaben des Pflegepersonals möglicherweise mit Angst, Nervosität, Schmerzen oder schlechten Erfahrungen in Verbindung bringen, ist jedoch nicht ungewöhnlich. Selbst wenn die routinemäßigen Aufgaben der Krankenpfleger*innen eigentlich schmerzfrei sind, können Kinder sich in Stresssituationen befinden, welche die Arbeit der Pflegekräfte deutlich beeinträchtigen. Nicht selten befindet sich entsprechend das zu behandelnde Kind, aber auch die behandelnde Pflegekraft, selbst bei einfachen, routinemäßigen Behandlungsmaßnahmen und trotz dem Einwirken der Eltern in einer anspruchsvollen Situation. Da das Pflegepersonal ohnehin wenig Zeit für zwischenmenschliche Interaktionen wie Gespräche mit den Patient*innen oder den Eltern hat, stellt sich die Frage, wie die Stresssituationen der Kinder und die damit verbundenen, herausfordernden Situationen der Pfleger*innen, unter Verwendung neuer technologischer Mittel, wie der Robotik, zukünftig verbessert werden können. Das Ziel der Untersuchung besteht darin, das emotionale Wohlbefinden von Kindern während der Pflegemaßnahmen mittels eines humanoiden Roboters und einer entsprechenden Anwendung positiv zu beeinflussen. Dabei wird dargestellt, wie der humanoide Roboter „Pepper“ (SoftBank Robotics Group Corp., 2020) während einer Pflegemaßnahme einen direkten Einfluss auf das Wohlbefinden von Kindern haben kann. Bereits zahlreiche Studien konnten den Mehrwert in der Interaktion zwischen Erwachsenen und Kindern mit „Pepper“ nachweisen und 
unterstreichen die vielfältigen Einsatzmöglichkeiten des Roboters, z. B. um das Lernen von Kindern zu fördern oder zur allgemeinen Unterhaltung (Tanaka et al., 2015; Aaltonen et al., 2017; Pandey \& Gelin, 2018).

\section{Ergebnisse der Befragung mittels Tablet-Anwendung und Fragebogen}

Um herzufinden, wie sich das Wohlbefinden und die Emotionalität der Kinder durch die Interaktion mit Pepper verändert wurde eine entsprechende Tablet-Anwendung, zusammen mit einer Gruppe Studierender der DHBW, entwickelt. In Abb. 5.1 ist Pepper zu sehen, welcher das Tablet auf der Brust fest installiert trägt. In Abb. 5.2 ist das Cover der Anwendung zu sehen, welches den internen Titel des Projektes trägt „Pepper Emotion Project“.

Die Anwendung dient im Versuch zum einen der Begleitung bei Interventionen aber auch der Abfrage über das Befinden des Kindes (siehe Abb. 5.3) vor und unmittelbar nach der Anwendung mit der Pflegekraft. Interventionen können dabei

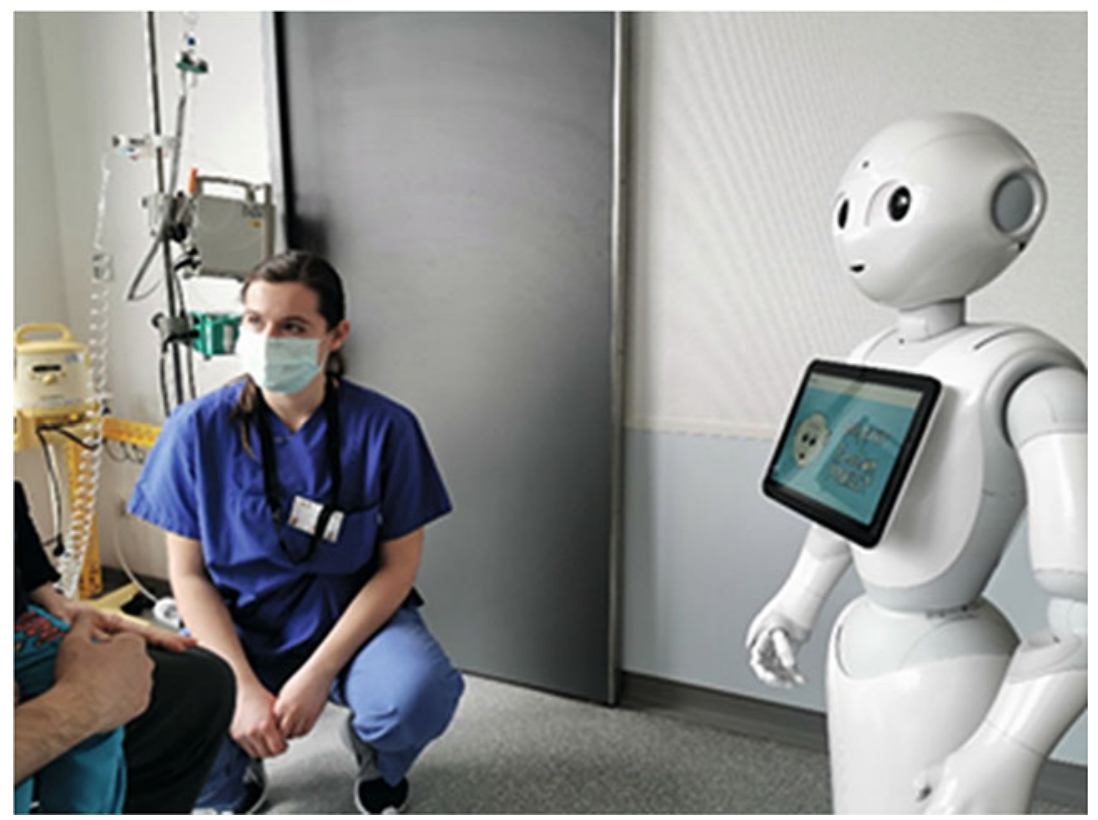

Abb.5.1 Die Pflegekraft steht in Kommunikation mit dem Kind, dem Elternteil und Pepper, und fungiert als Kommunikator zwischen allen Parteien 


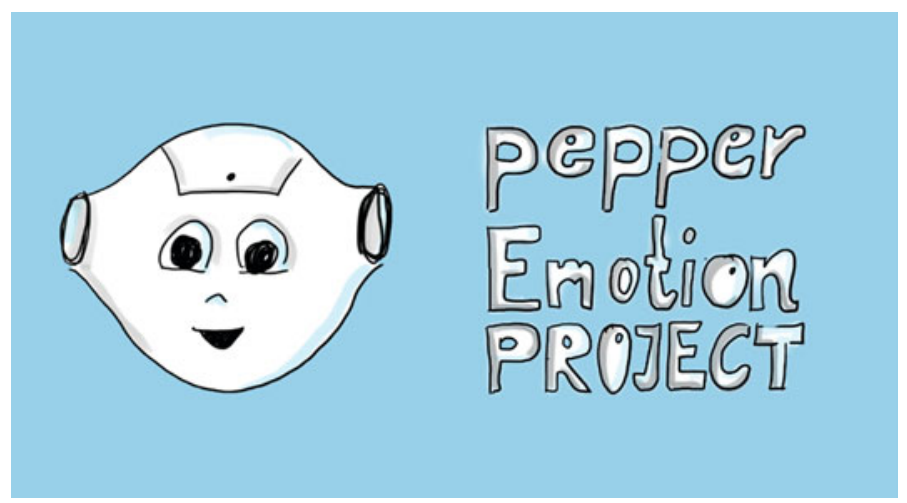

Abb.5.2 Das Cover der Anwendung mit dem Projekttitel „Pepper - Emotion Project“

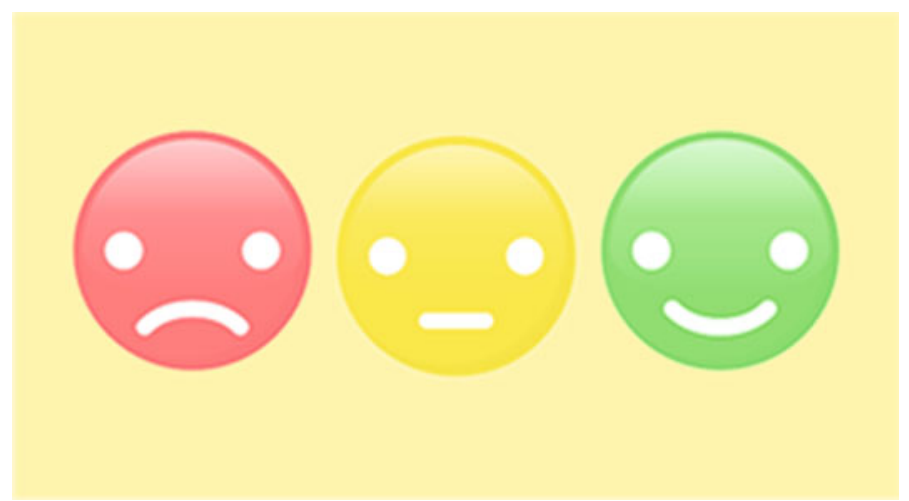

Abb.5.3 Symbole zur Bewertung des emotionalen Zustands

zum Beispiel Fieber und Blutdruck messen, Medikamentengabe, Verbandwechsel oder Inhalationen sein. Während dieser Anwendungen am Kind soll es durch Pepper in gewissen Maßen abgelenkt werden, um die Intervention schnell durchführen zu können, Ängste abzubauen, und das kindliche Stressniveau durch Vermeidung von Stress und Ablenkung zu senken.

Mittels dieser Darstellung konnten die Proband*innen vor und nach der Interaktion mit Pepper und der begleitenden Intervention ihren emotionalen Zustand angeben. Von links nach rechts bedeuten die Smilies „,sehr schlecht“, „gut“, ,,sehr 
gut“. Die Auswahl kann mittels verbaler Nennung der Begriffe erfolgen, oder per Touch über das Tablet.

Diese Ablenkung oder auch Begleitung konnte das Kind frei wählen. Pepper bot sprachlich, und mit der Anwendung untermalt verschiedene Aktivitäten wie zum Beispiel gemeinsames Singen, Memory spielen oder Tiere imitieren an.

Zur Vorbereitung einer Anwendung besteht die Möglichkeit kurze Videos auf verbalen Befehl hierzu anzusehen. Die Videos wurden selbst erstellt, und zeigen unter anderen den Ablauf der Inhalation oder auch den Verbandswechsel.

Als Pflegemaßnahme während des Experiments wurde bei zwei der Proband*innen eine Temperaturmessung durchgeführt und den weiteren Kindern konnte Pepper nur als allgemeine Ablenkung vom Krankenhausaufenthalt eingesetzt werden. Der Grund lag in der gesundheitlichen und pflegerischen Situation der Kinder.

Zusammensetzung der sieben hospitalisierten Proband*innen (Ergebnisse mittels Befragung der Eltern):

- 4 männlich; 3 weiblich

- 4 erstmalig hospitalisiert; $3>1$ Mal hospitalisiert (2 der 3 Probanden $<2$ Mal, 1 Proband $>2$ Mal)

- 2-11 Jahre alt

Die Proband*innen gaben mittels Sprache oder Tipp auf einen Smily auf dem Tablet, ihr aktuelles Befinden an.

Angaben zum Zustand vor der Intervention/vor der Interaktion mit Pepper:

- 5 von 7 Proband*innen: gut bis sehr gut

- 2 von 7 Proband*innen: nicht so gut

Angaben zum Zustand nach der Intervention/nach der Interaktion mit Pepper:

- 5 von 7 Proband*innen: gut bis sehr gut

- 1 von 7 Proband*innen: nicht so gut

- 1 von 7 Proband*innen: schlecht

Dem Proband, welchem es nach der Befragung schlecht ging hatte sich sehr bei der Interaktion mit Pepper über seine Kräfte hinaus engagiert, weshalb sich sein Zustand verschlechterte. Dennoch war er während der Interaktion vollkommen von seinen Schmerzen abgelenkt, und mobilisiert, was als Erfolg zu werten ist. 
Der emotionale Zustand der Kinder hatte sich im Kontrast zwischen der Befragung vor der Intervention und der Interaktion und danach nicht maßgeblich verbessert, oder blieb gleich. Der Grund für die geringe Veränderung lag mitunter an dem mehrheitlich sehr positiven Zusammentreffen von Pepper mit den Kindern. Bereits vor dem jeweiligen Versuch waren die Kinder erfreut über den Roboter, und auch unseren Besuch, was sie grundsätzlich eher positiv und freudig stimmte.

Neben den Kindern wurden auch die Eltern betreffend ihr Kind, der familiären Situation, der Erfahrung mit Klinikaufenthalten, dem Verhalten sowie der emotionalen Situation mittels Fragebogen befragt. Auch die Kinder sollten ergänzend zur Beantwortung über ihr Gemüt Fragen im Fragenbogen beantworten.

Antworten der Kinder betreffend der Einstellung gegenüber der Angst vor Pflegemaßnahmen:

- 1 von 7: eher selten Angst vor Pflegemaßnahmen

- 1 von 7: eher Angst vor Pflegemaßnahmen

- 5 von 7: nie Angst vor Pflegemaßnahmen

Antworten der Kinder betreffend die Frage wie oft sie ,,angstfrei“ bei der Interaktion mit Pepper waren:

- 1 von 7: immer angstfrei

- 1 von 7: ziemlich oft angstfrei

- 1 von 7: eher selten angstfrei

- 2 von 7: selten angstfrei

- 1 von 7: nie angstfrei

- 1 von 7: Kind hatte keine Fähigkeit zu antworten, aufgrund des sehr jungen Alters von 2 Jahren

Die Auswertung ergab, dass der Median ,eher oft“ bis ,eher selten“ ist, wenn man mit Unterstützung von Pepper über das Angstniveau der Kinder nach der Betreuungsmaßnahme spricht. Der Median ohne die Unterstützung von Pepper ist ,nie“.

\section{Ergebnisse der Beobachtungen}

Bei der Auswertung der Beobachtung der Interaktion mit Pepper wurden verschiedene Erkenntnisse gewonnen. Insbesondere bei der onkologisch erkrankten Patientin war die Freude über die Ablenkung durch Pepper deutlich erkennbar. Der Grund lag mitunter in der vergleichsweisen langen Zeit der Hospitalisierung 
gegenüber anderen Kindern von einigen Wochen, oder zahlreichen wiederkehrenden stationären Aufenthalten. Auch die Kinder der beiden anderen Klinikstationen waren über den Besuch und den folgenden Kontakt mit Pepper begeistert, jedoch in geringerem $\mathrm{Ma} ß$ als die onkologisch erkrankten Patientin.

Deutlich zeigte sich, je jünger die Proband*innen, insbesondere unter drei Jahren, waren diese gegenüber dem Roboter zu Beginn der Begegnung deutlich skeptischer, jedoch nicht abneigend oder verweigernd. Die Skepsis wurde durch eine erste Verweigerung der Kommunikation deutlich, und der Suche nach Blickkontakt mit den Eltern, was als Suche nach Schutz interpretiert werden kann.

Überwiegend konnte eine positive Beeinflussung des Gemüts der Kinder festgestellt werden, was sich durch Lachen und interessierten Blicken sowie Fragen zeigte. Positiv zu beobachten war entsprechend, dass die Mehrheit der Patient*innen zum Beispiel durch das Abspielen eines Videos so abgelenkt werden konnte, sodass Temperatur- und Blutdruckmessung ohne jegliche Störung möglich war. Zuvor zeigten unterschiedliche Patient*innen eher eine Abwehrreaktion auf die Pflegemaßnahmen.

Die positive und gemütsverbesserde Situation der Kinder trat insbesondere bei direkter, und funktionierender Interaktion wie Kommunikation mit Pepper auf. Wie auf Abb. 5.4 und 5.5 zu sehen ist, können sich die Kinder über den entsprechenden

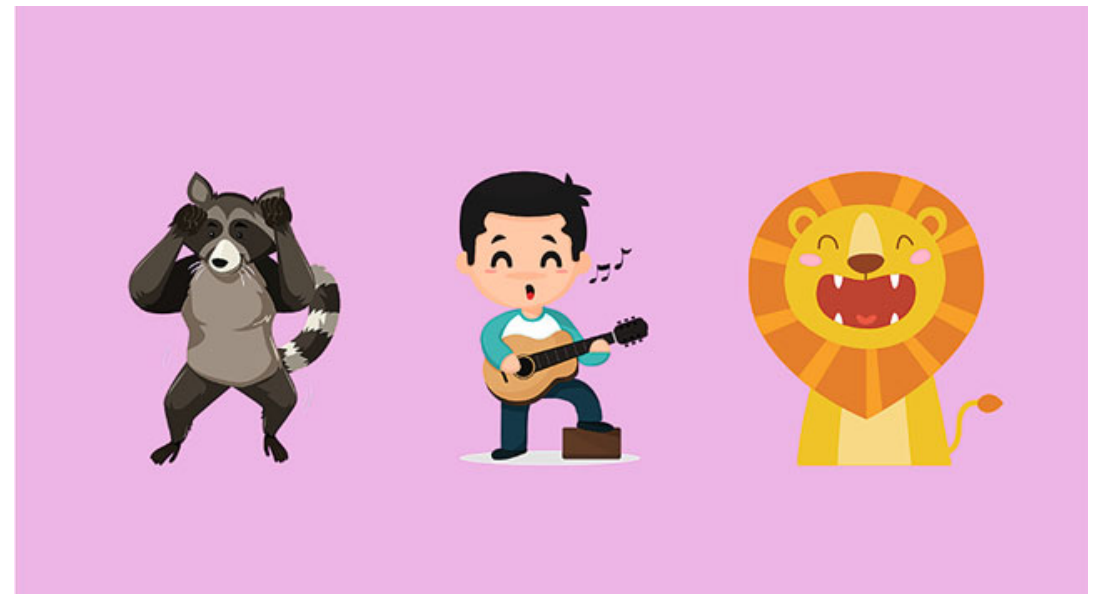

Abb. 5.4 Von links nach rechts steht die Auswahl zur Interaktion wie folgt zur Verfügung - „Memory“, „Singen“, „Tiere“ nachmachen. Die Auswahl kann mittels verbaler Nennung der Begriffe erfolgen, oder per Touch über das Tablet 

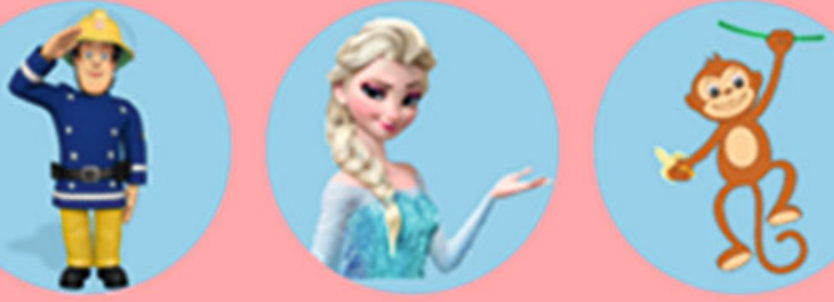

Abb.5.5 Wird „Singen“ gewählt, kann folgend das bevorzugte Lied zum Mitsingen ausgewählt werden. Auf dem Tablet wird ein passendes Video nach der Auswahl angezeigt. Die Auswahl kann mittels verbaler Nennung der Begriffe erfolgen, oder per Touch über das Tablet

Screen der Anwendung eine Interaktion mit Pepper auswählen.

Je intensiver die Kinder in die Interaktion eingebunden waren, und selbst aktiv waren, desto mehr erhellte sich das Gemüt, was durch Lachen, oder Grinsen zeigte.

Angebote von Pepper zur verbalen, handlungsorientierten oder sensorischen Interaktion, unter anderem über das Tablet von Pepper:

- Memory spielen über das Tablet

- Tiere imitieren (Pepper machte Bewegungen der Tiere unter Begleitung eines Bildes des Tieres auf dem Tablet vor)

- Gemeinsames Singen beliebter und allgemein bekannter Lieder, u. a. aus Filmen oder Kinderserien. Auf dem Tablet wurde ein entsprechendes Video gezeigt.

Die Möglichkeit, Tiere zu imitieren sowie Lieder zu spielen, wurde von fast allen Kindern gewählt, wobei die Proband*innen ab zehn Jahren selbst angaben, dass die bereitgestellten Interaktionsmöglichkeiten kein Interesse oder Begeisterung bei ihnen auslösen würde. Ältere Kinder, ab ca. 10 Jahren erlebten die größte Faszination und Ablenkung durch sprachliche Interaktion mit Pepper in Form von bilateralen Gesprächen, welche durch einen in die Anwendung integrierten und beschränkten Datenpool möglich war. 
Teilweise vergaßen die Proband*innen komplett Schmerzen oder unangenehme Berührungen, womit das Ziel Stress mittels Pepper zu reduzieren oder vollkommen zu eliminieren erreicht werden konnte.

War die Kommunikation zwischen dem Kind und Pepper jedoch gestört, zum Beispiel durch ein zu leises Sprechen mit Pepper, undeutliche Aussprache, oder deutlicher Akzent, sowie durch vermehrte Hintergrundgeräusche, sowie nicht ausreichend Licht für die Kameras des Roboters um zu fokussieren, so mussten Teile von Gesprächen oftmals wiederholt werden, die Eltern oder die Pflegekräfte alternativ in Kommunikation treten, oder sogar den Roboter wieder in eine optimale Position vor das Kind bringen da er sich abgewandt hatte.

Diese Komplikationen führten zu einer gestörten Kommunikation, und durch Korrekturen sowie durch Wiederholung zu einer deutlichen Verlängerung der Interaktion. Insbesondere bei kleinen Kindern war zu beobachten, dass dies einen großen Anspruch an die Konzentration der Patient*innen hatte und mit Unruhe, Stressanzeichen und Quengeln reagierten. Diese Überstimulation störte nicht nur die Kinder selbst, sondern mitunter auch die gesamte Untersuchung was auch die Auswertung der Interaktionen erschwerte. Daher gilt es für weitere Versuche derartige Störfaktoren aus der Umwelt zu reduzieren und möglichst auch die Proband*innengruppe definierter zu wählen.

Die Eltern sahen Pepper und die Interaktion mehrheitlich als positiv, und als hilfreiche Ablenkung an. Die Verhütung von Stresserleben und Reduktion steht bei ihnen im Vordergrund. Auch gab es die Aussage, dass Pepper unterhaltsamer war, als die bekannten Krankenhausclowns.

Die geplante Dauer einer Interaktion von 20 min wurde bei der eigentlichen Durchführung nicht eingehalten, da die einzelnen Interaktionen in der Regel nicht länger als 15 min dauerten.

\section{Zusammenfassung}

Fazit. Das Experiment befasste sich mit der Frage, inwiefern humanoide Roboter einen Einfluss auf das Wohlempfinden von Kindern während der medizinischen Behandlung haben kann und inwiefern ein Roboter als Assistenzsystem Pflegemaßnahmen verbessern kann. Ausgehend von der formulierten Hypothese, welche postuliert, dass es einen positiven Einfluss humanoider Roboter auf das Wohlbefinden von Kindern in der klinischen Behandlung gibt, konnten jedoch keine statistisch signifikanten Ergebnisse aufgezeigt werden, um die Hypothese annehmen zu können. Da die Auswirkungen von Pepper auf Patient*innen jedoch nicht nur anhand von Fragebögen, sondern auch mittels Beobachtungen erfolgte, kann festgehalten werden, dass Pepper insgesamt einen positiven Einfluss auf die Kinder und deren Emotionen hinterlassen hat. Dies zeigte sich z. B. durch plötzliches Lächeln oder 
ein entspanntes und ruhiges Verhalten der Kinder während der Behandlung, das vorher nicht vorhanden oder zumindest nicht offensichtlich erkennbar war.

Limitationen. Im Rahmen der Untersuchung sind einige Grenzen zu berücksichtigen. So muss beachtet werden, dass die Stichprobengröße nicht ausreichend ist, um statistisch signifikante Ergebnisse liefern zu können. Zudem wurden Kindern unterschiedlichen Alters innerhalb des Experiments als Proband*innen hinzugezogen. Es kann davon ausgegangen werden, dass eine Erhöhung der Stichprobe zu mehr Informationen über die Wirkungseffekte von Robotern auf das Wohlempfinden von Kindern während der Krankenpflege führt und auch unterschiedliche Effektstärken in Bezug auf Altersunterschiede zu erkennen sind. So könnte möglicherweise der Effekt von Robotern in der Krankenpflege altersabhängig sein, d. h. Kleinkinder könnten z. B. durch den Roboter beim ersten Kontakt erschreckt und verängstigt werden, während Schulkinder dagegen, aufgrund größerer Erfahrung mit der Technik, positivere Einstellungen haben könnten. Darüber hinaus gilt es zu beachten, dass negative Situationen mit Pepper u. a. auf die noch nicht immer ausreichend entwickelte Fähigkeit zur Interaktion zurückgeführt werden können, wenn das Kind z. B. undeutlich spricht, und Pepper dies nicht ganz oder gar nicht versteht und deshalb nicht darauf reagiert.

Implikationen. Auch wenn die Ergebnisse des Experiments aufgrund der Stichprobengröße keine umfangreichen Implikationen für Wissenschaft und Forschung zulassen, so kann dennoch der Aufbau des Experiments als Ansatz zur Messung eines direkten Einflusses eines humanoiden Roboters auf das Wohlbefinden von Kindern weiterentwickelt und verwendet werden. Auf der einen Seite zeigte sich dabei, dass Pepper eine positive, freudige Reaktion bei den Kindern schon vor Beginn der Untersuchung auslöste, als Sie den Roboter das erste Mal zu sehen bekamen. Auf der anderen Seite zeigte sich auch, insbesondere in den Pretests, dass der emotionale Zustand der Kinder von einer Vielzahl von Einflüssen abhängt und dieser sich im Laufe der Behandlungsmaßnahmen schnell verändern kann. Hierbei ist für zukünftige Forschungsarbeiten darauf zu achten, dass Kinder, abhängig von der gesundheitlichen Verfassung, der Akzeptanz gegenüber Technik im Allgemeinen und dem Alter, unterschiedlich auf Roboter reagieren können. Um diesen unterschiedlichen Einflussfaktoren zukünftig gerecht zu werden und um zu erfahren, welche dieser Faktoren letztlich zu einem positiven oder negativen Einfluss eines humanoiden Roboters auf das Wohlbefinden von Kindern führen, sollten weiterführende Studie über einen längeren Zeitraum durchgeführt werden. Hierzu kann das verwendete Untersuchungsdesign als Grundlage genutzt werden.

Ausblick. Das in dieser Untersuchung aufgeführte Experiment und die daraus abgeleiteten Ergebnisse machen deutlich, dass es im Themenbereich ,Robotik in der Pflege“" erheblichen Forschungsbedarf gibt. Zukünftige Forschungsarbeiten können 
dabei zur Verbesserung der Krankenpflege weiterführende experimentelle Ansätze liefern, welche einen quantifizierbaren und eindeutig statistisch-signifikanten Nachweis liefern, dass Roboter während Behandlungsmaßnahmen einen positiven Einfluss auf das Wohlempfinden von pflegebedürftigen Menschen nehmen.

\subsection{Development of the Acceptance of Robotics in Geriatric Care Under the Influence of Demographic Change}

Vorwort der Betreuer*innen: Pia Beyer-Wunsch, Christopher Reichstein

Um existierende und zukünftig auftretende Probleme im Hinblick auf den zunehmenden Pflegebedarf infolge des demographischen Wandels gerecht zu werden, beschäftigt sich die Wissenschaft und die Praxis seit Jahren mit Lösungen im Bereich Ambient/Active Assisted Living (AAL), insbesondere mit Fragestellungen rund um den Einsatz von Robotern. Einerseits muss bei der Verwendung von Robotik zwischen häuslicher und professioneller Pflege unterschieden werden, da die ambulante Pflege generell nicht mit der stationären Pflege verglichen werden kann. Andererseits hängt die Akzeptanz der Robotik in der Pflege allgemein von einer Reihe von Faktoren ab. Die vorliegende qualitative Untersuchung zeigt mittels einer strukturierten Literaturanalyse und einer Expertenbefragung, von welchen Faktoren die Akzeptanz von Pflegerobotern abhängen und liefert interessante Erkenntnisse sowie erste, fundiert hergeleitete Hypothesen, die im Umgang mit der Akzeptanz der Robotik im AAL-Umfeld zu berücksichtigen sind.

Stefan Besel, Daniel Bühner, Niklas Greilinger, Tim Hiller, Jasper Pecher, Wadim Sewostjanow, Leandra Sommer and Evelyn Wiebe

\section{Introduction}

In industrialized countries such as Germany, demographic change has been a topic frequently taken up and much discussed by the media and politics since years. In particular, the prevailing nursing care crisis will drastically intensify with a predicted population decline to 69 Mio. people by 2050 and due to an aging society (ZanderJentsch et al. 2019). Not only demographic but also digital change plays a major role in our society. More and more industries, including the care sector, are using more and more new technologies, such as robotics. Robotics helps to support and relieve the nursing staff and the people to be cared for. However, whether a nursing robot is actually accepted by the people concerned depends on numerous factors. In general, the question arises as to how the acceptance of robotics in care will develop, 
also under the influence of demographic change. In the first part of this paper, we will first define the relevant terms and then discuss various hypotheses on the topic, either verifying or disproving them by consulting the specialist literature and expert interviews. At the end of the paper the findings are summarized, and a conclusion is drawn.

\section{Definitions of Terms and Delimitation}

Acceptance. In general, acceptance describes the unrestricted willingness to accept a fact, a situation or a person (Endruweit et al., 2014). Thus, acceptance is an increase to mere tolerance, which does not necessarily have a positive effect. Acceptance is often described as "the characteristic of an innovation to achieve positive reactions from those affected by it when it is introduced (Endruweit et al., 2014).

The dictionary of sociology considers here the purely sociological aspect of acceptance. However, besides the general definition, acceptance is further subdivided into two dimensions. Especially a technical acceptance is defined differently. In the literature, it is essential to distinguish between two dimensions: the attitude and the action dimension (Schäfer \& Keppler, 2013). The attitude dimension comprises the affective and cognitive attitude towards a technology.

According to a study by the Center for Quality in Care (Eggert et al., 2018), in which 1000 German-speaking persons aged 18 and over from private households were questioned about the use of digital applications in care, $38 \%$ agreed with the statement that they quickly take a liking to new technical developments. The younger the people are, the more they like technical innovations. In this, respect in the next generations, there will be people in nursing homes who have a higher relation to technical innovations such as nursing robots. In a purely attitudinal understanding, acceptance means a positive attitude and assessment of the new technology and can also include an intention to act or a willingness to use the technology. However, it does not include actual action. The action-oriented view also includes the observable behavior of the application of a technology (Schäfer \& Keppler, 2013).

Robotics. Robotics deals with the design, configuration, control, production and operation of robots. These robots come in different shapes and designs depending on their task and capabilities. There are purely software-based robots, so-called bots, as well as physical robots. Humanoid robots, i.e. robots similar to humans, are now also being developed to make interaction with them more intuitive. The robots are slowly but steadily integrating into the living world of humans. In industrial manufacturing, robots have long been used for repetitive tasks and are now increasingly used for service tasks (Haun, 2006).

The sub-form of care robots supports human caregivers or carers and is available to those in need of care. They can perform physical tasks, such as helping to lie 
down and stand up, but also purely digital tasks, such as logging and reminding medication or alerting the emergency service. Some have natural language skills, are learning and intelligent systems (Bendel, 2019).

According to Bedaf et al. (2015) robots in care can be divided into three types. Robots for physical assistance, robots for social and emotional services and mobility aids. Robots for physical assistance typically combine navigation skills with sophisticated manipulation activities, so that they can help the patient with everyday tasks. In contrast to assistance robots, robots for social and emotional services do not focus on physical but on social-emotional support. Accordingly, interaction with objects is less important; rather, communicative and socially affective skills are required. The robot can serve either as an interaction medium or as an interaction partner. Mobility aids have the task of compensating for movement restrictions of the persons to be cared for (Bedaf, 2015).

Demographic change. Demographic change is leading to a change in the population structure. As a result of ever-improving medical care and living conditions, average life expectancy for women in Germany rose from 48.3 to 83.3 years, and for men from 44.8 to 78.5 years (Statistisches Bundesamt, 2019). Since the 1970s, Germany has been experiencing a trend towards an ageing society, which is the result of a decline in the birth rate and an ageing society. In all sectors, there are therefore not enough skilled workers available for the vacant positions. This means that there is also a discrepancy between nursing staff and those in need of nursing care. Between 2015 and 2035, the number of people in need of nursing care will increase by almost 1 million. This means that about 175,000 new nursing staff will be needed to cover the nursing requirements by 2035 (Kochskämpfer, 2018).

\section{Methodology and Data Collection}

At the beginning, we created a questionnaire for the upcoming interviews, based on the hypotheses developed previously by literature. For each hypothesis we derived a core question. The core questions were openly posed so that the interviewee would be able to give a far-reaching answer. The eight interviewees are experts (Tab. 5.1) working in research facilities of AAL and Living Labs and are, according to their research fields, proven experts in AAL. Two of the experts leads an AAL Living Lab, three are professors at universities and three are research assistants in the university environment. Due to the different professional backgrounds, a heterogeneous, interdisciplinary group of experts could be interviewed. This reflected their different views in areas of the questions. At the same time, they also showed large intersections in their experience and opinions on our questions in the field of AAL. 
Tab.5.1 Pseudonyms of interviewees, job positions and professional experience

\begin{tabular}{l|l|l}
\hline Pseudonym & Job Position & $\begin{array}{l}\text { Professional } \\
\text { Experience }\end{array}$ \\
\hline Manuel & Head of Division - Living Lab & Social Pedagogy \\
\hline Friedrich & Laboratory Engineer - Living Lab & $\begin{array}{l}\text { Computer Science Engineer in field } \\
\text { of health care }\end{array}$ \\
\hline Roland & Professor & $\begin{array}{l}\text { Business Information Technology } \\
\text { and Ethics }\end{array}$ \\
\hline Ludwig & Professor & $\begin{array}{l}\text { Care and rehabilitation management } \\
\text { and Human Machine Interaction }\end{array}$ \\
\hline John & Professor & $\begin{array}{l}\text { Care for the elderly } \\
\text { Care pedagogy } \\
\text { Gerontology } \\
\text { Nursing research }\end{array}$ \\
\hline Rita & Research Assistent & $\begin{array}{l}\text { Nursing Sciences } \\
\text { Nursing Management }\end{array}$ \\
\hline Maximilian & Research Assistent & $\begin{array}{l}\text { Business Information Technology } \\
\text { Human Machine Interaction } \\
\text { Social Robotic in Care }\end{array}$ \\
\hline Caroline & Research Assistent & $\begin{array}{l}\text { Medical Doctor } \\
\text { New technologies in care }\end{array}$ \\
\hline
\end{tabular}

In these facilities and Living Lab of the experts, experiments are being conducted with robotic systems for everyday care use. The systematizing expert interview took the form of a telephone conversation, which was recorded and transcribed with the consent of the interviewer. The aim was to obtain the most comprehensive possible knowledge of the experts' expertise with regard to the research topic (Bogner et al., 2014, S. 24).

The results were then analyzed and evaluated on the basis of the so-called "grounded theory" (Corbin \& Strauss, 1990). In the process, the experts' statements on each core question were coded. This means that the most important findings with regard to the question were filtered out and summarized in an overall result. The questionnaire with the 10 open questions for the semi-structured expert interview can be looked up in the appendix (Tab. A.1).

\section{Hypotheses}

In the following section, the hypotheses on the acceptance of robotics in geriatric care, which were established in the run-up to the study, are analyzed. For this 
purpose, a literature review and the survey of some experts in robotics and AAL were used. There is little or no empirical data in the literature. At most, individual applications can be found in experience reports. For example, experiences with the humanoid nursing robot "Pepper" and the seal "Paro" are the most frequent, but not statistically recorded. However, scenarios can often be found in which different robots can be used. Among the most frequently implemented scenarios for the use of nursing robots are water supply for the residents (Care-O-Bot 3), entertainment through games and memory trainers (Care-O-Bot 3 ), classic fetch and delivery services, e.g. of dirty laundry (service robotor called Casero 3 ) that monitors corridors during night service (Kehl, 2018). The experts confirm the knowledge gained from the literature in so far as the empirical values in practice are low, the nursing facilities usually only have knowledge of the existence of such nursing robots and the use of nursing robots has so far only taken place in a few facilities. Only a few tests have been carried out in such institutions. A reason for the poor experience is that the funding for research projects in the field of robots and care must be increased at federal and state level. An orientation towards Japan, which has been supporting companies and institutions in the field of care robotics for years with increasing subsidies, could be helpful here. In particular, the lack of a legal basis for remuneration makes care providers even more reluctant to use the new care technology (Fioranelli, 2019).

If one investigates the above-mentioned question of how widespread nursing robots are, the question inevitably arises as to whether a higher number of nursing robots will at the same time lead to greater acceptance of them. The following hypothesis can be derived from this question:

'Due to an increasing number of care robots in use, the acceptance of these robots is also increasing.'

Germany is one of the leading nations in the field of education in new technologies. In 2014 Germany was among the top 3 nations worldwide for robot patents (Robotic Launch Pad, 2014). The German social system and its institutions are well established, and modern technologies have been used and innovations developed for a long time. A trend towards the use of robotics in care is also confirmed by some of the experts we interviewed. The opinion here is that although there is a general trend towards the use of robotics in nursing care and a lot of research is being carried out and financing is being provided, a certain "hype" and skepticism cannot be denied. According to experts, acceptance can only develop if the added value of robotics is recognized and the effort for patients is kept to a minimum. If these prerequisites are met, it is undeniable that the acceptance of care robots will also increase with the increasing number of care robots used. The low acceptance of robots in care can be attributed, among other things, to the lack of technical experience of humans 
(Dijkers et al., 1991). Positive experiences already gathered can help to increase acceptance (Forlizzi, 2005). This leads to this hypothesis:

'Robots are more likely to be accepted by people with technical experience.'

Our experts have similar arguments. If the people have technical experience, they find it easier to handle the robots. Less inhibitions towards the robots make it easier to recognize their advantages. This is also expressed by Roland "anyone who has experienced such technology before will be able to handle care robots such as Lio and co. better" and continues "they will find it easier to use them. They are also more open to it.". The experts Maximilian and Ludwig also agree that "(...) experience with technology reduces the fear of contact with new technological devices. The barriers to use are then proportionately lower." However, the acceptance in this example still depends on several factors. If the robots are easy to use, the people do not have to learn much new things and can interact with the robots with their existing knowledge. Because they are less afraid of the technology behind the robots, they can be integrated more easily into the environment. It must also be noted that the acceptance often increases during the use of robots. This is where patients see the advantages of the robots and can get used to them (Koay et al., 2007). Experience already gained in the field of AAL has a similar influence on acceptance. People should generally be open-minded about the technology (Pfannstiel et al., 2018). If positive experiences with these technologies have already been gathered, this can increase acceptance. The following hypothesis is supported by these facts:

'People who already use AAL technology have a higher acceptance towards robots.'

The expert interviews show that experiences with AAL generally increase the acceptance of robots. If the advantages of AAL are recognized, these positive experiences with the technology can be transferred to the robots. Ludwig and John express that experience plays a major role. The more positive it was, the more there is an open-mindedness towards the new, like the robots. However, if robots show themselves to be susceptible to faults, the once positive image of technology is also disturbed. Rejection takes the place of the former acceptance. These experiences show real observations with the scope of robots. However, acceptance depends on how these initial experiences with AAL have gone. If problems have arisen here, it is possible that these people will associate the negative experiences with the robots. In addition, the effort required to operate the technology should be kept to a minimum and the real benefit of the technology should be in the foreground. In the literature there are no consistent classifications according to age with regard to the acceptance of care robots. According to the quantitative survey of persons aged 18 and over in the German population, Eggert et al. (2018) found that $44 \%$ of people in the age 
group of 60 years and older even refuse to deal with technical developments and $29 \%$ partially refuse to deal with them. This leads to the hypothesis:

'The older the human being, the more skeptical he is about social robots.'

Experts say that there are differences in age. Much more than age, the former profession has a decisive influence on the acceptance of a robot. The closer the profession was to technology, the more likely it is to be accepted. For example, Roland says that 74 years is not an old age, but when it comes to 80 years or 90 years, this makes it difficult to get close to the robot and to learn how to handle it. This even applies to people with former technical professions. Friedrich had similar observations. Furthermore, according to the experts, the recognizable benefit for the persons in need of care is also a big factor for the acceptance or rejection of care robots. Ludwig puts this in the point "and the important thing is that it is not the developer who sees a benefit in it (in the robot), but the user who sees a benefit. If the user can't see a benefit, then he puts it away and won't use it anymore." Regardless of age group, however, it can be assumed that the acceptance of new technologies tends to decline with age. Since it will take several years before robotic systems become more widespread in nursing care, acceptance will probably continue to rise for purely demographic reasons (Korn, 2019). Apart from age, experiences that the persons in need of care gain together with the carer with robotics seem to be essential for the acceptance of the latter. This leads to the following hypothesis:

'By gaining experience with robotics, together with the caregiver, the acceptance by those in need of care increases.'

In this field, a fundamental distinction must be made between the different types of robotics. There are already social robots which, accompanied by nursing staff, cheer up senior citizens in nursing homes - such as the learning robot seal "Paro" from Japan, which is already being used in several German homes. With this type of robot and with mobility-supporting robots, the joint gathering of experience with the nursing staff is decisive for the acceptance of those in need of care. This thesis is also supported by the interviewed experts: For them, an introduction with the nurse is indispensable, and technical support also plays an important role here. According to the experts, the presence of the nurse also leads to an increased feeling of security among those in need of care, which ultimately leads to a reduction in fear and skepticism towards the new technology. The experts Friedrich, Rita and Maximilian, among others, also carry this out. Maximilian also agrees with this, saying that the carers or social services are a "kind of catalyst" that get the people to be planted to deal with something. This accompaniment would also increase the "relationship of trust between the person in need of care and the nursing staff". This in turn leads to a greater willingness on the part of the people to be nursed to learn how to use the robot and to get to know it. 
Likewise, Ludwig, who believes that those to be cared for can better control "feelings such as fear or scepticism, as long as a real person is standing there beside them" and gives guidance. Furthermore, he explains "this is a big difference to (with accompaniment in contrast to without accompaniment) when humanoid robots enter a room alone. You have to be very, very careful, especially with people who have a cognitive impairment. This can lead to severe irritation or anxiety." Nevertheless, the acceptance behavior is individual and the training effort for caregivers should not be forgotten. In the case of assistance robots for physical everyday support, especially robots for transport and delivery services, i.e. those robots that are not necessarily in direct contact with the patients, the above-mentioned aspects are not decisive for acceptance by the introduction of a caregiver. In general, the technical approaches are accepted in all areas, although people like to keep certain distances between things but also to other people (Hall, 1966). Hall (1966) defines the different ranges as follows: The distances in public cover 3.7-7.6 $\mathrm{m}$ while the social distance reaches from 1.2 to $3.7 \mathrm{~m}$ and the personal distance from 47 to $122 \mathrm{~cm}$. Anything moving within these approximately $50 \mathrm{~cm}$ is called intimate distance. This leads to the following hypothesis:

'If the tasks are performed outside the intimate area of the patient, then the use of care robots is more likely to be advocated than for the tasks within the intimate area.'

According to our experts, the acceptance of robots can be increased if they operate outside the intimate sphere. Although there is usually an individual inhibition threshold, some people are afraid of losing human contact when a robot takes on intimate tasks. Roland says that the acceptance is very high if the robot is somewhere in the room or even behaves politely. LIO (a robot), for example, could knock on the door and wait to be invited in. He goes on to say that on the one hand, it can be problematic if the robot comes too close. On the other hand, the patients also want to touch the robot and be close. This shows the ambivalence in dealing with robots, as other experts also recognize. Manuel and Roland, also agree that it is not only a question of distance or intimacy, but also how much use and autonomy the robot actually brings about in effective ways.

It is important to discuss the different areas of action with the persons concerned in advance. The distance should only be reduced over time and the robot should not enter the intimate zone immediately. In addition, acceptance can be increased through self-determination. In this way, the person can decide for themselves which tasks the robot should or may perform.

All in all, the use of robots in intimate areas must therefore be discussed in detail, as acceptance problems often occur here. If this is clarified in advance and certain social norms are observed, acceptance of robots can be increased. This way robots 
can also take over tasks that take place within the intimate zone. In addition to that, there are differences in how the technology works. Although data protection or data security plays a less important role for people, camera systems tend to be rejected. If a robot enters the intimate zone of a patient it should be clear how it collects data and what kind of data it collects (Braun et al., 2016). The main concern of those affected is the fear of losing interpersonal contact when robotics replaces human assistants. Loneliness is already an issue for many elderly people living alone and could be exacerbated by robotics (Bendel, 2019). These concerns lead to the following Hypothesis:

'People in need of care become lonely due to the use of social robots, due to the lack of human interaction.'

Many of our experts also saw this danger. However, some of them also reported that the use of robots in groups could even promote social behavior. In relation to this, Maximilian notes that the handling and the tendency to be lonely is related to programming and further explains "Basically, we have seen that when we use social robots in groups, interaction takes place in the group. One talks together about the robot." The robot can be used as a board-object, and thus relieve loneliness or even contact shy. This view is also shared by Rita, who adds that it is not absolutely necessary to place the robot in some communication with a patient, but that group use is also possible. This would also encourage the patients to leave their rooms and participate in other common activities. A social added value is created.

Ludwig points out that the problem of loneliness, especially at home, is nothing new. This is to be regarded in particular, if domestic animals are actually purchased against isolation. This happens when the animal is the only communication partner and increasingly replaces human communication. Of course, this must not happen, neither with pets nor with robots. So, it is not possible to make a general statement about this hypothesis, because the effects are always individual. Certainly, there is the possibility of loneliness if the robot completely replaces a human being who has been there before. But if the robot is used as a support and the social contact is passed on, for example by a group, there is no danger of social isolation. There are even robots that actively work against the problem of loneliness, such as the Alice doll developed in the Netherlands (Becker, 2018, S. 234).

The support of care robots is most frequently used in hospitals, followed by the retirement home and home use. Surveys show, however, that more and more senior citizens have a positive attitude towards the use of caregiving robots. For example, in a representative Forsa survey carried out in April 2016 on behalf of the BMBF, $83 \%$ of those surveyed could basically imagine using a service robot in their own homes in old age in order to be able to live there longer (Forsa, 2016). We worked out the following hypothesis: 
'The acceptance of robots in the patient's home is higher than in a care facility.'

Our experts also confirm this. According to them, acceptance increases if this allows for longer autonomy in the home environment. Correspondingly, John states that it could be argued that "in the home environment, I had bought it (the robot) myself, so you could assume that the acceptance is greater. If a care facility acquires such a robot, I am usually not asked." Which ultimately reduces acceptance.

Friedrich and Rita agree that in addition to the decision for the robot, it is also necessary to consider what is to be done with the acquisition costs, who can and wants to carry it, especially with the still high prices. In addition, it must be clarified who will maintain the robot safely in which environment and how quickly users can contact someone if problems arise with it. Is this better possible at home or in the vicinity of a nursing home? However, some of them also noted that many challenges, such as cost, accessibility, getting to know the robot and maintenance of the robot, make it difficult to implement in the home environment. There is also still too little experience, so that patients cannot be given good recommendations. In the future, however, this could still change and our experts all saw great potential for care robots in the home environment for the future. Other studies by Broadbent or Wu already have shown that older people prefer discrete, small robots with humanlike or pet-like behavior to large humanoid robots (Broadbent, 2009; Wu, 2012). So, we came to the hypothesis that:

'The abstract machine-like models in front of the humanoid models are chosen as sympathetic.'

Our experts also think the appearance of the robot is very important and said that it is crucial for acceptance. It should not be too human, but at the same time it should not have too many technical elements, as these can lead to fear. Our experts tended to prefer a rather childish and simple appearance. However, it would always depend on the subjective assessment of the patients. Some experts also noted that it is not only the outward appearance that matters, but also the voice and age of the robot. Since it should also be possible to build up an emotional bond with the robots, appearance is one of the first and most important factors that influences acceptance.

The preferred appearance of social robots is influenced by culture, as well as by personal preferences, which, however, are increasing. For example, the expert Roland explains, "Most social robots tend to be white, have a plastic surface, seem somehow clean, neat, likeable, and that's an important point," and regarding the voice, he says, "Pepper's usual voice, the childlike voice, is not necessarily appropriate in a nursing context. That's not really where children should be". They don't necessarily trust the children in the care context either. So the voice is extremely important. Pepper's voice matches his appearance, it is small. It should be noted that Pepper is also less of a caregiver than a social entertainer. Nevertheless, the voice 
is very relevant for acceptance. Roland and John agree that the robot is not scary. This happens when the robot is too human, too powerful, and by a human-like size too massive and therefore threatening.

\section{Conclusion}

As part of the qualitative study design, experts on AAL were interviewed to clarify questions about the development of the acceptance of robotics in geriatric care under the influence of demographic change. It is important to note that the acceptance of robotics in geriatric care depends on a number of factors, which can be influenced and positively changed by correct handling. Essential for the future success of robotics in care and the acceptance of patients is also the right training of the nursing staff because they are essential for the introduction and as contact persons for the people to be cared for. Despite the lack of experience and the low level of use in institutions, it can be said that there is a trend in robotics (Kehl, 2018). The experts agree that acceptance is growing among many through personal experience as well as trial and error. Whether the age really makes a difference with respect to the acceptance of robotics could not be conclusively clarified by the experts. Robotics in nursing is not a vision of the future, but a technology that on the one hand already exists and is being used, but on the other hand also needs to be further developed and researched.

The results of the present research work are primarily intended to shed light on the extent to which people who are in care institutions accept robots as help. Ethical aspects were not considered in the present work, initially due to the restricted scope. It must be mentioned that the data on grounded theory obtained through expert interviews were evaluated, but not with the intention of developing independent theories or even a conceptual model, but only to derive hypotheses which can be verified or falsified in a subsequent study, for example by means of a quantitative study design. As long as robots are not used extensively in practice, it is difficult for science to carry out quantitative research. However, this is also due to the fact that health insurance companies in Germany have so far not covered any costs for robots. Only when health insurance companies are prepared to at least partially cover the costs of robots will more robots be used in nursing care, and only then can science conduct quantitative and qualitative research on robots in nursing care.

Due to demographic change, future generations will find it easier and almost natural to receive interactive help, including support from robots. Maybe it will not be the next generation yet, but at least the generation after next. Then it will also be possible to use more robots in practice under this aspect. From a scientific point of view, more values and key figures will then be available. 


\subsection{Akzeptanz von Sensoren zur Erfassung medizinisch relevanter Vitalparameter im und am Körper: Horrorvorstellung oder Hoffnung?}

\section{Vorwort des Betreuers: Volker Andelfinger}

Die Entwicklungen im Rahmen des Internet der Dinge (IoT, Internet of Things) bringen auch Sensoren zur Erfassung von Vitalparametern hervor, die dem Körper immer näherkommen und auch im Körper implantiert werden können. Die Entwicklung ist einerseits noch am Anfang und die Sensoren werden zukünftig viel mehr wichtige Parameter messen können, andererseits ist es Zeit, sich mit der Frage zu befassen, wie weit Menschen diesen Weg der kompletten Vermessung und Datennutzung vor dem Hintergrund ethischer Fragestellungen mitgehen wollen, mithin ob derartiges vertretbar, gewünscht, akzeptiert ist. Der folgende Beitrag ist nicht explizit auf eine AAL-Thematik hin verfasst, ist jedoch inhaltlich übertragbar, die Fragestellung ist eine generelle.

Marco Egetenmeyer, Kai Horlacher, Lukas Lutz, David Ollmann

\section{Einleitung}

Im Zeitalter der Digitalisierung sind auch im Gesundheitswesen neue Technologien auf dem Vormarsch, die versprechen die Gesundheit erheblich verbessern zu können. Im Bereich von eHealth spricht man dabei unter anderem von verschiedenen Sensoren, die sowohl im Körper als auch am Körper getragen werden können. Einige Technologien, wie zum Beispiel der Herzschrittmacher oder die automatische Insulinpumpe, werden bereits flächendeckend eingesetzt. So ist Deutschland eines der Länder mit den meisten Herzschrittmacher-Implantationen weltweit. Die Zahlen haben sich dabei in den letzten Jahren auf einem hohen Niveau stabilisiert. (IQTIG-Institut, 2017). Auch die Zahl der Diabetes-Patient*innen, welche automatische Insulinpumpen zur Regelung des Insulinspiegels im Körper nutzen, steigt stetig an. Von über 300.000 Diabetes Typ 1 Patient*innen wurden bereits ca. 50.000 einer Insulinpumpentherapie zugeführt. Dementsprechend nutzt ca. jeder sechste der Erkrankten die automatische Insulinversorgung (Siegel \& Siegel, 2019).

Im Bereich der Mikrochiptechnologien gibt es einige Modelle, die einen großen Nutzen versprechen. Jedoch sind wenige Technologien bekannt, die bereits in der breiten Masse verwendet werden. In diversen Studien wird hingegen gezeigt, dass die Akzeptanz bzw. die Bereitschaft sich einen Mikrochip für medizinische Zwecke implantieren zu lassen in den letzten Jahren gestiegen ist. So waren es 2016 lediglich $15 \%$ der befragten Menschen in Deutschland (Rohleder, 2016). Dieser Wert hat sich binnen 2 Jahren auf $32 \%$ im Jahr 2018 mehr als verdoppelt (Zühlke, 2019). Ob diese Umfragewerte die tatsächliche Bereitschaft zeigen sich einen Mikrochip 
zu implantieren, ist jedoch fraglich. Dadurch kommt es zu einer Verzerrung der Realität und der gegebenen Antwort in der Umfrage (Bortz \& Döring, 2006). Den Schätzungen zufolge sind aktuell lediglich ca. 3000 Menschen in Deutschland mit einem Mikrochip ausgestattet (Leistner, 2018).

In dieser Arbeit soll die Frage geklärt werden, warum digitale, medizinische Implantate und Wearables trotz steigender Akzeptanz im medizinischen Alltag bisher kaum zur Anwendung kommen. Dabei stellt sich die Frage, welche Hindernisse und Probleme der Technologie dafür sorgen, dass sich bisher sehr wenige Menschen dafür entschieden haben.

\section{Sensoren im und am Körper}

Im Folgenden sollen Technologien dargestellt werden, die im oder am Körper getragen werden können und zur Verbesserung des Gesundheitszustands beitragen. Zum einen gibt es Implantate, die in den Körper eingesetzt werden und zum anderen gibt es Wearables, die am Körper getragen werden können.

\section{Wearables}

Wearables sind Geräte, die für eine längere Zeit am Körper getragen werden können. Es handelt sich um Sensoren, die sich auf der Haut befinden. Mit diesen Sensoren, die in den letzten Jahren häufig in Smartwatches, oder auch sogenannte smarte Pflaster integriert wurden, können verschiedene Vitalparameter wie Herzfrequenz, Atemfrequenz, Blutdruck und Körpertemperatur gemessen werden. Des Weiteren kann beispielsweise ein Elektrokardiogramm erstellt werden (Isakadze \& Martin, 2019). Mithilfe dieses EKG können u. a. Herzrhythmusstörungen frühzeitig erkannt werden. Das EKG lässt sich anschließend an den Hausarzt übermitteln, wodurch Anzeichen für Schlaganfälle erkannt werden können. Ein vollwertiges medizinisches EKG kann es jedoch nicht ersetzen. Andere Wearables haben eine Funktion zur automatischen Sturzerkennung. Dabei erkennt die Uhr, wenn der Träger einen Unfall hatte oder gestürzt ist. Wenn der Benutzer nach einer gewissen Zeit nicht reagiert wird automatisch der Notruf kontaktiert. Gerade für ältere Menschen, die alleine wohnen, kann diese Funktion von großem Nutzen sein. Weiter gibt es sogenannte Biosensoren, die sowohl direkt auf die Haut aufgeklebt oder in der Kleidung getragen werden können. Umgangssprachlich spricht man dabei oft von SmartenPflastern (Agarwal, 2018). Diese Art von Sensoren ermöglichen den Ärzt*innen das Auslesen und Beobachten von bestimmten Vitalparametern der Patient*innen. So kann beispielsweise die Bewegung, die Körpertemperatur oder der Herzschlag beobachtet werden. Zudem ermöglichen Wearables die Messung der Sauerstoffsättigung im Blut. Hierzu wird der Unterschied zwischen dem sauerstoffreichen und sauerstoffarmen Blut bestimmt. Das Ergebnis gibt somit an, wie viel Prozent der 
roten Blutkörperchen (Hämoglobin) mit Sauerstoff gesättigt sind (Giersch et al., 2019). Weiter kann durch das Messen der Herzfrequenzvariabilität (HRV) angegeben werden, wie hoch das Stresslevel der Person ist. Dabei wird die Änderung der Zeit zwischen zwei Herzschlägen ermittelt. Ziel ist das Vorbeugen von Folgeerkrankungen, sowie chronischem Stress (Humbert, 2019). Die Aussagekraft der Messungen ist bisher allerdings fraglich, da Einflussfaktoren wie Alkohol, Nikotin und Übergewicht die Ergebnisse beeinflussen können (Schimpl, 2017).

\section{Implantate}

Im Gegensatz zu den Wearables werden Implantate in den Körper eingepflanzt. Die Sensoren bzw. Geräte befinden sich unter der Haut, weshalb sich das Ein- und Ausschalten bzw. das Abnehmen dieser Technologien im Vergleich zu den Wearables schwieriger gestaltet. Beispielsweise könnten implantierte Mikrochips als Arzneimittelpumpe dienen und in Zukunft die Medikamentenversorgung im Körper regeln. Über Jahre hinweg könnten die Implantate Medikamente dosiert an den Körper abgeben. Durch die Automatisierung könnte das Leben chronisch kranker Menschen erheblich erleichtert werden. Probleme wie vergessene Pilleneinnahmen und falsche Dosierungen würden der Vergangenheit angehören (Salmen, 2015). Ein Beispiel dafür ist die implantierte Insulinpumpe. Diese bestimmt den Insulingehalt im Blut und gibt dementsprechend eine bestimmte Dosis automatisch an den Körper ab, ohne dass der*die Patient*in aktiv etwas tun muss. Auch Personen die an Osteoporose oder Parkinson erkrankt sind, könnte damit geholfen werden (Farra et al., 2012).

Eine Alternative wäre die Nutzung von Mikrochips als Ersatz oder Erweiterung der Health Tracking Wearables. Viele Menschen nutzen bereits Smartwatches oder Pulsuhren, um Gesundheitsdaten wie Blutsauerstoffgehalt, Temperaturprofil, Herzfrequenzmuster und Atmungsmuster aufzuzeichnen. Dies kann in Zukunft mit Mikrochips geschehen mit dem Vorteil, dass die Qualität der Daten in Vergleich zu den Wearables zunehmen könnte. Die Gesundheitswerte können über Apps für den Nutzer einsehbar sein und ihm somit regelmäßige Updates über seine Gesundheit geben (Schwartz, 2019). Entwickelt man diese Idee weiter, dann könnte der Mikrochip im Falle von kontinuierlichen kritischen Werten wie dem Bluthochdruck, Ärzt*innen oder sonstige medizinische Versorger alarmieren. Somit könnte Folgeprobleme wie Schlaganfälle entgegengewirkt werden. Oder aber die Gesundheitsdaten bleiben lokal auf dem Mikrochip gespeichert und dienen als eine Art Gesundheitspass, der alle relevanten wichtigen Gesundheitsdaten einschließlich Allergien enthält und zur Information der Ärzt*innen in Notfällen dient (Trager, 2019). 


\section{Kritische Betrachtung \\ Probleme und Risiken}

Die Chancen und Risiken bei Sensoren im und am Körper liegen eng beieinander. Sie können kritische Gesundheitswerte ein Leben lang speichern und damit genaue Auskünfte über den Gesundheitszustand des Nutzers geben. Dabei stellt sich die Frage, ob dieser technologische Fortschritt mehr persönliche Freiheit schafft, oder ob er sie einschränkt. Die Probleme, die in diesem Abschnitt betrachtet werden, beziehen sich auf die Technologie selbst und damit auf die Datensicherheit, Manipulation und Funktionalität.

Bereits heute lassen sich mit dem Smartphone Bewegungsprofile erstellen. Als Erweiterung ermöglichen Mikrochiptechnologien zusätzlich die Aufzeichnung von Vitalparametern. Beispiele hierzu ist die Überwachung der Körpertemperatur, des Sexuallebens, der Essgewohnheiten und des Schlafs. Zum einen können diese Daten ein Vorteil bei der eigenen gesundheitlichen Kontrolle sein. Zum anderen können die Daten als Druckmittel vom Arbeitgeber, von Versicherungen, oder auch Kriminellen eingesetzt werden, wenn sie in die falschen Hände gelangen (Tretbar, 2017). Neben dem Überwachungsrisiko bieten die Mikrochips auch einige Sicherheitsrisiken. Sie können von Betrügern ausgelesen werden, was von Sicherheitsfirmen bereits mehrfach demonstriert wurde (Moutafis, 2019). Dabei wurde mittels NFC-Technik ein Handy über den implantierten Chip gehalten, sodass der Hacker die Daten des jeweiligen Nutzers auslesen konnte (Frankfurter Allgemeine Zeitung, 2018). Auch die Datenintegrität spielt dabei eine Rolle. Wenn die Daten von Hackern ausgelesen werden können, dann besteht die Gefahr der Manipulation der Daten. So könnten kritische Gesundheitsdaten manipuliert werden, was zu schwerwiegenden Folgen führen könnte (Smith, 2008). Zudem lässt sich sagen, dass der durchschnittliche Träger solcher Technologien über die Funktionsweise nicht ausreichend informiert ist. Die Daten werden teilweise in Clouds, oder bei externen Dienstleistern gespeichert. Zum einen wissen die Nutzer nicht, ob die Daten Ende-zu-Ende verschlüsselt kommuniziert werden. Zum anderen ist die Verarbeitung, Speicherung und der Zugriff auf die Daten keineswegs transparent gestaltet (Kurz, 2016). Zusammenfassend kann gesagt werden, dass der Verlust der Privatsphäre durch Datenklau oder Manipulation ein Risiko für den Nutzer darstellt. Die gesundheitliche Belastung von Mikrochips im Körper ist dagegen noch nicht ausreichend erforscht. Vor allem bei Chips, die regelmäßig und lange am Körper getragen oder sogar implantiert werden, ist es sehr wichtig zu untersuchen, welche Langzeitwirkungen daraus resultieren. Tierversuche machen deutlich, dass es durchaus einen Zusammenhang zwischen implantierten Chips und Krebs geben könnte (Rötzer, 2007). Um eine Gesundheitsgefährdung ausschließen zu können, müssen die Untersuchungen in diesem Zusammenhang fortgesetzt werden. Ein weiteres Problem sind die Daten, die auf den 
medizinischen Produkten erhoben und gespeichert werden. Fraglich ist hierbei, wie genau die Daten sind und wie gewährleistet werden kann, dass eine allgemeine medizinische Verwendung stattfinden kann. Ärzt*innen dürfen Werte aus solchen Chips nur verwenden, wenn sie medizinisch zertifiziert sind. Hierzu bedarf es einer CEKennzeichnung. Um ein solches Kennzeichen zu erhalten, müssen entsprechende EU-Richtlinien des Medizinproduktegesetzes eingehalten werden. Allerdings sind bisher nur wenige Produkte mit diesem Qualitätszeichen ausgestattet (MEDCERT, 2020).

\section{Ethische Probleme}

Die bereits thematisierten Risiken und Probleme, wie der Verlust der Privatsphäre, die Datensicherheit oder auch der Missbrauch von Daten, sorgen sicherlich für einen großen Teil der Skepsis gegenüber Chip-Implantaten. Es wirkt jedoch beinahe ironisch, dass die Menschen in einer Welt, in der nahezu jeder Mensch unzählige persönliche Daten in den sozialen Medien von sich Preis gibt, die Angst vor dem Verlust der Privatsphäre bemängeln (Schwartz, 2019).

Es scheint so, als seien den Menschen ihre persönlichen und individuellen Vitalparameter wichtiger als die Lifestyle-Daten, die sie in den Sozialen Medien preisgeben. Die Angst vor dem Verlust oder Missbrauch der Gesundheitsdaten durch Arbeitgeber oder auch Krankenkassen ist zu groß, denn dabei kann es um existenzielle Entscheidungen gehen und nicht nur um Lifestyle-Fragen. Dementsprechend ist nur jeder zweite deutsche Bundesbürger bereit allen Ärzt*innen und Krankenkassen die persönlichen Gesundheitsdaten digital anzuvertrauen (PWC, 2019). Für Diskussionsstoff sorgt dabei das Stichwort der Datenhoheit. Da es sich um die persönlichen medizinischen Daten der erkrankten Person handelt, sollte die Datenhoheit bei ihm liegen. Er selbst sollte entscheiden können was mit seinen Daten geschieht, wer sie einsehen darf und wie genau sie verarbeitet werden. Ärzt*innen kritisieren dabei, dass sie nie einen vollständigen Überblick über die Gesundheit ihrer Patient*innen bekommen würden. Jedoch würde den Patient*innen durch die Bevormundung durch einen Arzt die Kontrolle über ihre persönlichen Daten entzogen, was nicht in deren Sinne sein kann (Kötter, 2017). Es wird also deutlich, dass die ethische Vertretbarkeit in dieser Thematik auch eine große Rolle spielt, denn auch ethische Bedenken fallen immer mehr ins Gewicht und sorgen für die Skepsis der Menschen. Als ethisches Hauptproblem wird immer wieder genannt, dass sich Mikrochips nicht auf oder an der Haut, sondern unter der Haut befinden. Die Implantate verbinden sich mit der Materie des Körpers und können im Gegensatz zu Wearables nicht kurz abgelegt werden. Dazu werden medizinische Eingriffe benötigt. Damit einhergehend sind die Menschen in einem hohen Maße von den Herstellern der Chips abhängig, wenn sie repariert, aufgeladen, ausgetauscht oder 
entfernt werden sollen (Michael \& Michael, 2005). Des Weiteren herrschen auch Unklarheiten in Diskussionen um den Entscheidungsträger von Implantationen bei Minderjährigen oder psychisch kranken Menschen. Auch der Aspekt der sozialen Gerechtigkeit führt immer wieder zu kontroversen Diskussionen. Es muss die Frage geklärt werden, wie der Zugang zu solchen oft sehr teuren und personalisierten Technologien sozial gerecht gestaltet werden kann, da Krankenkassen nur selten die hohen Kosten übernehmen (Gasser et al., 2019). In diesem Zusammenhang muss auch das Thema Zwang zur Implantation beachtet werden. Mikrochips dürfen nie die einzige Alternative für den Menschen sein. Es sollte immer eine andere Möglichkeit zur Lösung des entsprechenden medizinischen Problems existieren, denn jeder Mensch sollte letztendlich selbst entscheiden können ob er ein Implantat nutzen möchte (Riedl, 2019). Im Zeitalter der Digitalisierung mit Teilgebieten wie künstliche Intelligenz und Big Data scheint es nur eine Frage der Zeit zu sein bis Mikrochips in der Lage sein werden nicht nur simple Entscheidungen wie beispielsweise die Menge der Medikamentenabgabe zu treffen, sondern auch komplexe Entscheidungen übernehmen. Wann werden auf Basis der getrackten Gesundheitsdaten auch ethische Entscheidungen, beispielsweise über Leben und Tod getroffen (Andelfinger \& Hänisch, 2016)? In diesem Zusammenhang lässt sich feststellen, dass sich die Skepsis und damit das mangelnde Vertrauen mehr auf die Institution, die die Technologie kontrolliert bezieht, als auf die Technologie selbst. So wird von vielen Kritikern behauptet, dass die Institution, sei es der Staat oder ein privates Unternehmen, früher oder später darauf aus sein wird den Menschen zu kontrollieren (IP et al., 2008). Bevor die Mikrochiptechnologien in der breiten Masse eingesetzt werden können, besteht ein Klärungsbedarf all dieser Fragen.

\section{Nutzenpotential}

Wie bereits beschrieben stiften die medizinischen Sensoren im und am Körper durchaus einen Nutzen für den Anwender. Für jeden der beschriebenen Anwendungsfälle gibt es jedoch auch herkömmliche Lösungen, die weniger komplex sind. Deshalb müssen die neuen digitalen Technologien einen Mehrwert versprechen, der ausreichend groß ist, um die damit verbundenen Risiken zu rechtfertigen.

Ein Beispiel, in dem ein derartiger Mehrwert erzielt wird, ist der Einsatz von vollautomatischen Insulinpumpen. Im Vergleich zu herkömmlichen Insulinpumpen erfolgt die Blutzuckermessung als auch die entsprechende Insulinabgabe automatisch. Bei herkömmlichen Pumpen sind Blutzuckermessung und Insulingabe getrennte Funktionen. In dem Closed-Loop System der automatischen Pumpen sind Pumpe und Sensor miteinander vernetzt. Durch die automatische Blutzuckermessung und Insulingabe wird de*m*r Patienten*in lebensnotwendige Aufgaben 
abgenommen und somit Lebensqualität geschenkt. Darüber hinaus sind die Insulinwerte im Vergleich zu herkömmlichen Pumpen genauer und befinden sich öfters im Zielbereich (Hüttemann, 2018). Diese immense Erleichterung im Alltag kann dazu führen, dass potenzielle Nutzer*innen über die genannten Risiken hinwegschauen. Auch im Bereich der Wearables sind derartige Modelle vorhanden. Die sogenannten Patches, die auf die Haut geklebt werden und mit einem Sensor ausgestattet sind, können sowohl Vitaldaten messen als auch zur Medikamentierung eingesetzt werden. So können Vitaldaten in medizinischer Qualität erhoben werden. Über Sensoren können die Daten an die Ärzt*innen übermittelt werden, wodurch Krankheiten bereits in einem viel früheren Stadium als jetzt erkannt werden können. Auch die Langzeitüberwachung bei chronischen Krankheiten wie Alzheimer wird vereinfacht. Ärzt*innen haben die Vitaldaten im Blick und können in kritischen Situationen eingreifen, wohingegen sie bisher nur punktuell Zugriff auf die Daten hatten. Durch die Mehrzahl an gewonnenen Daten können personalisierte Therapien entwickelt werden. Darüber hinaus kann der Einfluss von verschiedenen Lebensstilen und Umweltfaktoren auf Krankheiten erfasst werden (Kirschenbauer, 2018). Einen weniger großen medizinischen Mehrwert bieten immer noch sehr viele der Smartwatches. Diese können zwar den Puls messen und noch weitere interessante Daten erfassen, haben allerdings keinerlei medizinische Zertifizierung. Das bedeutet im Umkehrschluss, dass die Daten für Ärzt*innen unbrauchbar sind. Aber auch hier ist ein Wandel festzustellen. Smartwatch-Produkte von Apple (Apple, 2019) oder Withings (Withings, 2020) besitzen mittlerweile entsprechende Zertifizierungen zur Messung von EKGs oder Sauerstoffsättigung.

\section{Wie kann die Akzeptanz gesteigert werden?}

Die Basis für eine breite Akzeptanz, sowohl von Implantaten als auch von Wearables, ist eine ausgereifte Technologie, die im Vergleich zu den herkömmlichen medizinischen Mitteln einen deutlich erkennbaren Mehrwert bietet. Dabei muss für die Nutzer*innen klar erkenntlich sein, welche Daten erhoben werden und welches konkrete Problem durch die jeweilige Technologie gelöst werden kann. Ohne die Lösung eines konkreten Problems werden die Nutzer das Risiko der digitalen Überwachung nicht eingehen. Grundsätzlich wichtig dafür ist ein transparentes und sicheres Datenmanagementkonzept. Transparent heißt, dass es für den Nutzer deutlich ersichtlich ist, wo seine Daten gespeichert werden, wer darauf zugreifen kann und wie die Daten darüber hinaus verarbeitet werden. Dabei sollte die Datenhoheit aufseiten der Verbraucher liegen. Sie selbst müssen entscheiden können wer die Daten einsehen kann und wie die Daten verarbeitet werden (Rövekamp, 2019). Essenziell wichtig dafür sind Regulierungen und Gesetze, die den Datenmissbrauch bereits präventiv vorbeugen und bei Verstoß konsequent bestrafen sollen. 
Internationale und landesspezifische Gesetze müssen die Sicherheit, Vertraulichkeit, Konsistenz und Integrität der persönlichen Daten gewährleisten (Smith, 2008). Die genannten Regulierungen und Gesetze sollen von einer Institution erlassen werden, die den Fokus auf die Verbraucher*innen und Anwender*innen legt. Es sollte sich um eine Institution handeln, die zwischen den Anwendern und den Unternehmen, die hinter den eigentlichen Technologien stehen, vermittelt. Dabei muss die Unabhängigkeit zu den Unternehmen weitestgehend gewahrt werden. Staatliche Regulierungen sind zudem wichtig, um die ethischen Streitigkeiten zu klären. Dazu sind weitere Gesetze und Regulierungen notwendig, die beispielsweise regeln wie die Vergabe sozial gerecht gestaltet werden kann und welche Anlaufstationen es bei Problemen gibt. Darüber hinaus dürfen Menschen, die diese Technologien nicht nutzen möchten nicht benachteiligt werden (Smith, 2008). Weiter ist die Verwendung einer standardisierten Zertifizierung als Zulassung der Technologie als Medizinprodukt notwendig. Dieses Siegel bzw. Zertifikat sollte an qualitativ hochwertige Produkte vergeben werden, die medizinisch verwendbare und verwertbare Daten erheben. So kann gewährleistet werden, dass die Technologie genaue Daten, die von Ärzt*innen ausgewertet können, erhebt. Werden unverlässliche Daten geliefert, dann wird die Nutzung deutlich beeinträchtigt, da qualitativ schlechte Daten erhoben werden die zu medizinischen Fehleinschätzungen führen können. Als Beispiel kann hierbei das Europäische CE-Zertifikat genannt werden (MEDCERT, 2020). Unabhängig von der Genauigkeit können Arztbesuche nicht ersetzt werden. Die Daten können aber dazu genutzt werden, um auf notwendige Arztbesuche hinzuweisen (Nagel, 2019).

\section{Fazit}

Im Rahmen dieser Ausarbeitung sollte untersucht werden, wieso medizinische Wearables und Implantate in Deutschland bisher wenig genutzt werden, obwohl die Akzeptanz laut einigen Umfragen deutlich höher ist. Um diese Frage beantworten zu können, wurde eine ausgiebige Literaturanalyse durchgeführt. In mehreren Gruppendiskussionen wurden zudem eigene Beiträge erarbeitet, die mit in diese Ausarbeitung eingeflossen sind. Zunächst müssen die zu Beginn erwähnten hohen Umfragewerte relativiert werden. Menschen neigen dazu, dass sie in Umfragen Entscheidungen treffen, die sie in der Realität nie treffen würden. Daraus kann man schließen, dass Menschen sich eher gegen die Implantation von Sensoren entscheiden würden, wenn Sie diese Entscheidung konkret treffen müssten. Das liegt hauptsächlich daran, dass der Nutzen der implantierten Sensoren noch nicht groß genug ist, um die Risiken zu überwiegen. Bei den sogenannten Wearables sieht das jedoch anders aus. Sie werden im medizinischen Umfeld noch nicht flächendeckend eingesetzt, finden aber in der Öffentlichkeit eine höhere Verbreitung, weil dort auch 
der private Nutzen höher ist. Beispielsweise ist hier die Smartwatch zu nennen. In Zukunft werden die genannten Technologien auch im medizinischen Umfeld immer mehr verbreitet sein. Die Basis dafür ist, dass der Nutzen im Gegensatz zu den herkömmlichen medizinischen Lösungen erhöht wird und durch staatliche Rahmenbedingungen gewährleistet werden kann, dass die Nutzer beim Einsatz solcher Produkte nicht geschädigt werden.

Ob die Nutzung der digitalen Implantate und Wearables zur Horrorvorstellung oder Hoffnung werden bleibt abzuwarten. Durch die bereits genannte staatliche Regulierung und auch Zertifizierungen kann das Szenario der Horrorvorstellungen vermieden werden. Wird zusätzlich der konkrete Nutzen erhöht, können Sensoren im und am Körper durchaus zur Hoffnung in der Zukunft werden. Das medizinische Personal kann entlastet werden und Krankheiten können zugleich früher erkannt bzw. besser behandelt werden. Die damit verbundenen Probleme und Risiken dürfen jedoch nicht außer Acht gelassen werden. Letztendlich muss jeder Mensch die Entscheidung für sich treffen, ob er bereit ist die Risiken einzugehen und dadurch eine Erleichterung im Alltag zu erlangen.

\subsection{Telemonitoring in der Kardiologie am Beispiel von Patient*innen mit Herzinsuffizienz}

Vorwort des Betreuers: Marcel Sailer

Herz-Kreislauferkrankungen können sich rasch zu lebensbedrohlichen Gefährdungen entwickeln. Eine Rückmeldung über die Herzfunktion sowie eine Sicherheit der begleiteten Beobachtung ist für die Menschen von (lebens)entscheidender Bedeutung. Telemonitoring kann dabei eine Hilfestellung bieten, für Sicherheit in der häuslichen Umgebung zu sorgen und schnell auf kritische Situationen zu reagieren. In großen Ländern mit weiten Anreisen zur medizinischen Versorgung (z. B. USA, Australien u. a.) sind Telemedizinstrukturen seit vielen Jahren etabliert. Vitalparameter zur Herzleistung (z. B. Herzrhythmus, Herzfrequenz), Blutdruck- und Sauerstoffregulation werden hier kontinuierlich gemessen und in den Versorgungsprozess einbezogen. Der vorliegende Artikel analysiert literaturgestützt die technologischen und strukturellen Möglichkeiten für das deutsche Gesundheitssystem.

Anna Raible, Anika Ludwig, Alissa Bittinger, Fynn Zeisele, Uwe Muell, Luca Fischer, Alex Baciu, Martin Kost 


\section{Einführung}

Der Bereich Telemonitoring in der Medizin befasst sich mit der Fernüberwachung von Patientendaten. Es besteht mittels digitaler Übertragung von diagnoserelevanten Daten ebenfalls die Möglichkeit zur Fernuntersuchung und -diagnose Stellung zu nehmen. Die gesammelten Daten der Patient*innen im Rahmen einer abgestimmten Therapie können folglich in Echtzeit oder im bestimmten Turnus von medizinischem Fachpersonal überwacht und abgerufen werden. Man unterscheidet nicht-invasives Telemonitoring von invasivem Telemonitoring. Das nicht-invasive Telemonitoring kann aktiv vom Patienten durchgeführt und übermittelt werden und erfordert keine Implantate. Beispiele für nicht-invasives Telemonitoring sind telemedizinische Waagen, Blutdruckmessgeräte und EKG-Geräte (Bundesärztekammer, 2019, S. 127).

Invasives Telemonitoring fordert bestimmte Implantate, welche teilweise über chirurgische Eingriffe eingesetzt werden, beispielsweise zur Übertragung von Daten zum Herzrhythmus oder Blutdruck. Bei einer Überschreitung von Grenzwerten können diese am angeschlossenen Netzwerk einen Alarm auslösen und es kann zeitnah in das Geschehen eingegriffen werden (Bundesärztekammer, 2019, S. 1).

Telemonitoring bietet eine Möglichkeit den drohenden Problematiken des demografischen Wandels und dem Mangel an Ärzt*innen in ländlichen Regionen entgegen zu wirken und den Ausblick einer erleichterten Datenerhebung zu Forschungszwecken bei Volkskrankheiten (Bundesärztekammer, 2012). Die Herzinsuffizienz ist in Deutschland die häufigste pathologische Einweisungsdiagnose ins Krankenhaus. Nach dem ersten Krankenhausaufenthalt des herzinsuffizienten Patienten kommt es bei fast $30 \%$ der Patient*innen innerhalb eines Jahres zur Rehospitalisierung (Freund et al., 2016, S. 229-246).

Bei einer Herzinsuffizienz ist die Leistung des Herzens herabgesetzt und das von Körper benötigte Blutvolumen kann nicht mehr adäquat gefördert werden. Hypertonie, Aortenstenosen oder entzündliche Herzerkrankungen können unter anderem Ursachen einer Herzinsuffizienz sein. Die ersten Symptome zeigen sich in der Regel mit einer Gewichtszunahme, peripheren Ödemen, Tachykardie und einer Belastungsdyspnoe bis hin zur Ruhedyspnoe (Becker, 2015, S. 96). Diese Parameter könnten mittels Telemonitoring erfasst und an den medizinischen Begutachter gesendet werden. Bei einer Verschlechterung der Erkrankung wäre so vor drohenden Komplikationen ein therapeutisches Einschreiten möglich. Bei einem implantierten Herzschrittmacher mit einem ICD oder CRT System besteht die Möglichkeit des Auslesens des Herzrhythmus um beispielsweise bei Arrhythmien (Herzrhythmusstörungen) oder einem Ausbleiben des Herzschlages direkt ein Warnsignal an den behandelnden Kardiologen oder eine verknüpfte Rettungsleitstelle zu senden mit der angezeigten Dringlichkeit zur Behandlung. Der Bereich der Telemedizin bietet 
einige Chancen aber auch Risiken im Bereich der medizinischen Behandlung und Betreuung von Patient*innen. Weitere Vorteile sind eine optimierte Begleitung und Betreuung der Betroffenen, denn es besteht die Möglichkeit in einem Notfall schnell zu reagieren und somit das Sicherheitsgefühl der Patient*innen zu erhöhen. Zudem können durch Telemonitoring Langzeitdaten für eine Verbesserung der medizinischen Therapie gewonnen werden. Die Patient*innen erhalten durch die Daten Feedback zu ihrem Alltagsverhalten und können dadurch in Belastungsmomenten autonom reagieren.

\section{Methodik}

Im Folgenden wird die Anwendung von Telemonitoring Systemen in der Kardiologie analysiert. Für ein systematisches Vorgehen wurde die Methodik der Literaturanalyse mit anschließender kritischer Bewertung der Potenziale im Bereich Telekardiologie gewählt. Somit können verschiedene Kriterien aus der Theorie genauer beleuchtet und dadurch ein möglichst breiter Bereich abgedeckt werden. Neben den technischen Möglichkeiten im Bereich des Telemonitoring werden Chancen und Risiken für das Gesundheitssystem, Nutzen für die Patient*innen, Notfallversorgung sowie Datenschutz und Finanzierung untersucht. Durch die interdisziplinäre Zusammenarbeit von Studierenden aus dem Gesundheitsbereich und der Wirtschaftsinformatik konnten die verschiedenen Kriterien fachspezifisch aufgearbeitet werden. Mittels Literaturrecherche u. a. in den Datenbanken Pubmed, Medline und Cochrane library, Springer Link, EDS und EMBASE werden die Kriterien zunächst in einer Analyse objektiv dargestellt und anschließend kritisch beleuchtet.

\section{Ergebnisse}

Die Ergebnisse der Literaturanalyse werden nun zunächst objektiv anhand der vorgestellten Kriterien dargestellt. Die kritische Bewertung seitens der Autor*innen erfolgt im darauffolgenden Abschnitt, der Diskussion.

\section{Technische Möglichkeiten}

Im folgenden Abschnitt werden die unterschiedlichen Ansätze bzw. technologischen Möglichkeiten, die es zum heutigen Standpunkt zur Echtzeitüberwachung der Parameter von Patient*innen mit Herzkrankheiten gibt, dargestellt und miteinander verglichen. Telemedizinische Monitoring Systeme werden momentan bereits in einzelnen regionalen Projekten oder von kommerziellen Anbietern eingesetzt und dabei entsprechend ihrer Funktionsweise unterschieden. Dabei erfolgt eine Klassifizierung anhand des jeweiligen Informationsflusses, welcher von der Generierung 
einer diagnostischen Information bis zur Einleitung der erforderlichen medizinischen Maßnahme reicht. Die drei unterschiedlichen Generationen lassen sich dabei wie folgt einteilen (Köhler \& Lücke 2007, S. 110-111):

Systeme der 1. Generation: Zeichnen mittels Sensoren Ereignisse von Patient*innen auf und schicken diese, beispielweise per SMS, ohne eine weitere Verarbeitung direkt an den behandelnden Arzt. Bsp.: Implantierbare KardioverterDefibrillatoren (ICD).

Systeme der 2. Generation: Dauerhafte Überwachung von Patient*innen mittels Sensoren und Echtzeitüberprüfung von Fachpersonal in Servicecentern (dabei handelt es sich i. d. R. nicht um Ärzt*innen). Bei relevanten Abweichungen vorher festgelegter Grenzwerte wird der betreuende Arzt vom Servicecenter kontaktiert. Sollte dieser nicht erreichbar sein, so können die Angestellten des Servicecenters auf andere verfügbare Ärzt*innen ausweichen.

Bsp.: telemedizinisches Gewichtmonitoring bei Herzinsuffizienz

Systeme der 3. Generation: Individuelle Zusammenstellung der medizinischen Messgeräte anhand der jeweiligen Bedürfnisse der betroffenen Person. Die gesammelten Messwerte treffen in einem rund um die Uhr ärztlich besetzen Telemedizinischem Zentrum (TMZ) ein. Bei Bedarf erfolgt anschließend eine Kontaktaufnahme mit dem Patienten oder behandelnden Arzt und Notfallmaßnahmen können ergriffen werden. Die Verantwortung liegt hierbei beim behandelnden TMZ Arzt, wodurch Verzögerungen vermieden werden. Dieser kann entsprechend des vorliegenden Notfalls Dosierungsänderungen vornehmen oder den Notarzt alarmieren. Zusätzlich erfolgt eine permanente Echtzeitüberwachung der Parameter, welche die regelmäßigen Visiten des Patienten beim Hausarzt ergänzen. Bsp.: Patient*innen mit fortgeschrittener chronischer Herzinsuffizienz und kurzen Entscheidungsintervallen bis zur Einleitung therapeutischer Maßnahmen. Die Klassifikation der unterschiedlichen Telemedizinischen Generationen entspricht dabei keiner technologischen Unterlegenheit der ersten und zweiten Generation. Die Eignung der einzelnen Systeme wird dabei durch die Schnelligkeit des Handlungsbedarfes bei den unterschiedlichen Patient*innen determiniert (Köhler \& Lücke, 2007, S. 110-113).

\section{Die Chancen und Risiken für das Gesundheitssystem}

Telemonitoring bei Herzinsuffizienz bietet sowohl Chancen als auch Risiken für das Gesundheitssystem. Welche damit gemeint sind, wird nachfolgend näher beleuchtet. Die Bundesärztekammer sieht im Bereich der Telemedizin durchaus Chancen diese weiter zu etablieren. Aus ärztlicher Sicht ist es notwendig die Versorgungsfelder durch Telemonitoring festzulegen und zu definieren. Durch das große Anwendungsgebiet in der Telemedizin besteht die Möglichkeit, den drohenden Problematiken 
durch den demographischen Wandel und dem Ärztemangel in ländlichen Regionen entgegen zu wirken (Bundesärztekammer, 2012).

Das Ziel ist eine aktive Gestaltung der telemedizinischen Entwicklung und Anwendung seitens der Ärzt*innen. Der primäre Fokus der Telemedizin liegt dabei auf der Verbesserung von Patientenversorgungsstrukturen, an sekundärer Stelle stehen ökonomische Optimierungen (Bundesärztekammer, 2012). Es ist zu beachten, dass eine Echtzeitübertragung der Patientenparameter nur dann gründlich begutachtet werden kann, wenn ausreichend geschultes Fachpersonal zur Verfügung steht und sowohl technische als auch arbeitsstrukturelle Abläufe angepasst werden. Die Organisation und Finanzierung dieser Strukturen zum flächendeckenden Einsatz von Telemonitoring stellt das Gesundheitssystem vor Herausforderungen. Telemonitoring ist als Ergänzung zu regelmäßigen Arztbesuchen zu verstehen, um drohende Verschlechterungen des Patienten frühzeitig zu erkennen und intervenieren zu können. Somit könnten Krankenhausaufenthalte verhindert werden, was wiederum Kosten und Ressourcen spart und dadurch die Kliniken und Krankenkassen entlastet (Bundesärztekammer, 2019, S. 128). Durch telemedizinische Versorgung eröffnet sich auch die Möglichkeit für behandelnde Ärzt*innen den bürokratischen Aufwand zu verringern und den Fokus auf die Patiententherapie zu setzen. Dies wäre eine Steigerung der Versorgungsqualität. Prognosen zu Folge wäre eine Kostenverringerung im Gesundheitssektor durch Telemonitoring durchaus möglich. Vor allem bei chronischen Erkrankungen und Erkrankungen des Herz-Kreislaufsystems ist eine Steigerung der Versorgungsqualität gewährleistet, welche Kostensenkungen in diesem Bereich mit sich bringt. Laut dem statistischen Bundesamt fielen 2017 46,4 Mrd. EUR für chronische Erkrankungen und Herz-Kreislauferkrankungen an, diese entsprechen 13,7 \% der gesamten Gesundheitsausgaben (Statistisches Bundesamt, 2017). Die randomisierte, prospektive, multizentrische, gesundheitsökonomische Studie EuroEco $(\mathrm{N}=303)$, welche sich mit der Fernüberwachung von ICD-Patient*innen befasst konnte eine Kostenreduktion beobachten. Dies ist auf die geringere Anzahl der Hospitalisationen bei Patient*innen, welche unter Telemonitoring in Behandlung sind, zurückzuführen. (Heidbuchel et al., 2015). Die Unternehmensberatung McKinsey veröffentlichte eine Analyse, welche ein deutliches Nutzenpotential der Teleberatung durch die Fernüberwachung von 7,7 Mrd. EUR aufweist (McKinsey \& Company, 2018). Es sind folglich durchaus Einsparungen durch telemedizinische Verfahren zu erwarten, bezüglich verkürzter Krankenhausaufenthalte und gesteigerter Lebensqualität der Patient*innen. Dennoch wird es vor allem anfangs eher zu einer Kostenverschiebung wirtschaftlicher Ressourcen als zu einer Kostenreduktion kommen. 


\section{Nutzen für die Patient*innen}

Im folgenden Abschnitt wird das Kriterium der Patientenperspektive im Sinne der Krankheitsbewältigung im Alltag analysiert. Beleuchtet wird dies anhand einer Metaanalyse. Kotb et al. (2015) haben sich in einer systematischen Überprüfung und einer Metaanalyse mit verschiedenen Studien hinsichtlich ihrer Wirksamkeit verschiedener Formen der Telemedizin für Patient*innen mit Herzinsuffizienzen beschäftigt. Von den Datenbanken: MEDLINE, EMBASE, CINAHL und Cochrane Library konnten 30 Studien ( $=10.193$ Patient*innen) verwendet werden. Dabei wurden Studien eingeschlossen, die über das primäre Ergebnis der Mortalität oder eines der folgenden sekundären Ergebnisse berichteten. Dazu zählen allgemeine Krankenhausaufenthalte und Krankenhausaufenthalte aufgrund einer Herzinsuffizienz (Kotb et al., 2015). Bezüglich des Nutzens für die Patient*innen gingen folgende Ergebnisse aus der Metaanalyse hervor: Im Bereich der allgemeinen Sterblichkeit zeigte sich, dass diese durch Telemonitoring und eine strukturierte telefonische Unterstützung zurückging. In Bezug auf Patient*innen mit einer Herzinsuffizienz zeigte sich ein deutlicher Rückgang der Zahl der Krankenhausaufenthalte im Vergleich zu einer üblichen Versorgung. Zusammenfassend hat diese Analyse gezeigt, dass eine strukturierte telefonische Unterstützung und Telemonitoring-Maßnahmen von erheblichem Nutzen für die Rehabilitation von Herzinsuffizienz-Patient*innen sein können (Kotb et al., 2015). Allerdings weist die Metaanalyse auch darauf hin, dass weitere Forschungsarbeiten notwendig sind, um die Langzeitauswirkungen von Telemonitoring auf die Betroffenen zu untersuchen.

Eine konkrete Studie, welche sich mit dem Nutzen von Telemonitoring bei Patient*innen mit chronischer Herzinsuffizienz beschäftigte, ist die TIM HF2 Studie der Charité in Berlin von Koehler, Koehler, Deckwart et al. 2016. Dabei wurde die Hälfte der 1538 Proband*innen mit chronischer Herzinsuffizienz mit einem Elektrokardiografen (EKG) mit Fingerclip zur Messung der Sauerstoffsättigung, einem Blutdruckmessgerät, einer Waage und einem Tablet zur Selbsteinschätzung des Gesundheitszustandes ausgestattet (Koehler et al., 2018). Die Studienergebnisse zeigen, dass die telemedizinisch mitbetreuten Patient*innen weniger Tage durch ungeplante Einweisungen aufgrund von Herzinsuffizienz im Krankenhaus verbringen mussten: im Durchschnitt waren es 3,8 Tage pro Jahr im Vergleich zu 5,6 Tagen pro Jahr in der Kontrollgruppe. Damit haben die telemedizinisch betreuten Patient*innen insgesamt und bezogen auf die einjährige Studiendauer pro Patient signifikant weniger Tage durch ungeplante kardiovaskuläre Krankenhausaufenthalte verloren oder sind in dieser Zeit verstorben d. h. 17,8 Tage im Vergleich zu 24,2 Tagen in der Kontrollgruppe, was einer Reduktion von ca. $26 \%$ entspricht. Darüber hinaus wies die telemedizinische Gruppe eine signifikant geringere Gesamtsterblichkeit im Vergleich zur Kontrollgruppe auf (Koehler et al., 2018). 
2007 beschreiben Köhler und Lücke bereits in „Partnership for the Heart: Klinische Erprobung eines telemedizinischen Betreuungssystems für Patient*innen mit chronischer Herzinsuffizienz", dass eine Diskrepanz zwischen objektiver Verschlechterung und subjektiver Wahrnehmung einer verschlechternden Herzfunktion besteht. Mit geeigneter telemedizinischer Betreuung wird im Idealfall die beginnende objektive Verschlechterung diagnostiziert, bevor der Patient sie wahrnimmt (Köhler \& Lücke, 2007, S. 112-113).

\section{Notfallmanagement}

Der folgende Abschnitt beschäftigt sich mit Telekardiologie in der Notfallmedizin. Schwerpunkt der Analyse stellt die Art der Datenübertragung und deren Auswertung dar. Zudem werden die Konsequenzen der Auswertung begutachtet. Während der Literaturrecherche zeigte sich, dass die meisten Studien in Bezug auf das Telemonitoring von kardiologischen Patient*innen vermehrt auf die Prävention von kardiologischen Dekompensationen ausgelegt sind. Für konkrete Notfallsituationen werden meist keine genauen Handlungsabläufe aufgeführt.

2005 wurde von Zugck, Nelles und Frankenstein (Zugck et al., 2005, S. 178) eine kontrollierte prospektive Studie aufgrund eines kardiologischen Telemonitoring Programms der Personal HealthCare Telemedicine Services durchgeführt. Hierbei wurden 270 Patient*innen im Stadium II-IV nach NYHA konstant telemedizinisch überwacht. Der Patient übermittelte in diesem Programm vorgegebene Vitalparameter per Telefon an das telemedizinische Zentrum. In diesem werden vorab individuelle Grenzwerte gespeichert und bei einer Überschreitung bzw. Unterschreitung der Grenzen ein Alarm ausgelöst und therapeutische Maßnahmen eingeleitet. Zusätzlich wurden die Patient*innen unabhängig der Vitalparameter proaktiv kontaktiert und befragt. Bei kardiopulmonalen Symptomen oder ernsthaften Beschwerden können die Patient*innen zu jedem Zeitpunkt das telemedizinische Zentrum erreichen (Zugck et al., 2005, S. 179-182). Die Ziele hierbei waren die medikamentöse Compliance zu fördern und Veränderungen des Gesundheitszustands der Patient*innen frühzeitig zu erkennen. Es zeigte sich, dass in einem Zeitraum von drei Monaten 150-mal ein Alarm ausgelöst wurde, wobei sich nur bei $2 \%$ der Fälle ein akuter ärztlicher Handlungsbedarf zeigte. Das Telemedizinische Zentrum wurde von den Patient*innen 93-mal notfallmäßig wegen akuten Kardio pulmonalen Beschwerden kontaktiert. In 2 Fällen war daraufhin ein Notarzteinsatz erforderlich und 18 der Patient*innen wurden an ihre behandelnde Klinik oder ihrem behandelten Arzt verwiesen (Zugck et al., 2005, S. 179-182).

Das Studienprotokoll der Telemedical Interventional Management in Heart Failure (TIM-HF II), welches bereits erwähnt wurde, zeigt ein ähnliches Modell des Monitorings, dieses wurde bereits im Kriterium des Nutzens für Patient*innen 
erläutert. Die Messwerte werden per Mobilfunk an das Telemedizinische Zentrum übermittelt und anhand von festgelegten Parametern, IT-gestützt in fünf Kategorien eingeteilt (Koehler et al., 2018, S. 1047-1057). Bei Abweichungen von den Parametern sind sechs verschiedene Eskalationsmaßnahmen festgelegt, diese reichen von einer telefonischen Kontaktaufnahme bis zu einer Notarztalarmierung (Koehler et al., 2018, S. 1048). Außerdem ist das Telemedizinische Zentrum befugt die Feuerwehr oder Polizei zu verständigen (Koehler et al., 2018, S. 1056), sollte es keinen Kontakt zum Patienten oder Angehörigen herstellen können.

Es zeigte sich bei der weiteren Literaturrecherche, dass es im Notfallmanagement unterschiedliche Systeme gibt. Jene befinden sich meist noch in Testphasen, weshalb auch das Vorgehen bei einer Abweichung der Daten von Normwerten noch nicht einheitlich geregelt ist (Krüger-Brand, 2016, S. 15-17).

\section{Datenschutz}

Die wachsende Vernetzung im Sinne der Digitalisierung stellt für alle Beteiligten im Gesundheitswesen neue Anforderungen dar. Dabei gewinnt der Umgang mit Datenschutz und Datensicherheit eine ansteigende Bedeutung (Krüger-Brand, 2016, S. 218). Aus diesem Grund wird im folgenden Abschnitt die Bedeutung des Datenschutzes in Bezug auf die Übertragung von Patientendaten im Fokus des Telemonitoring analysiert.

Neue gesetzliche Regularien wie das IT-Sicherheits- und E-Health-Gesetz, sowie die europäische Datenschutzgrundverordnung veranlassen eine Veränderung im medizinischen Bereich. Vor allem bei Telematik-Strukturen zeigt ein vergangenes Ereignis im Dezember 2019, bei dem zahlreiche, persönliche Daten von Ärzt*innen, Patient*innen und Versicherten missbraucht worden sind (Tutt, 2020), dass technische und organisatorische Maßnahmen erfolgen müssen, um die bis 2021 zuwachsenden Gesundheitsdaten von mehr als 70 Mio. Versicherten und Patient*innen in einem angemessenen Rahmen zu schützen (Tutt, 2020).

Insbesondere Telekardiologie erfordert einen höchstmöglichen Schutz, da sensible Daten übertragen werden, die das menschliche Leben gefährden können, sollten sie in falsche Hände geraten. Dabei geht es um die Online-Kommunikation, mit der zum Beispiel Geräte wie Herzschrittmacher Vitalparameter an Ärzt*innen und Therapeut*innen senden. Allerdings besteht auch die Möglichkeit bei Implantaten mit einer Defibrillator-Funktion, Signale beispielsweise über Funkwellen zu manipulieren, was lebensgefährliche Folgen für die Patient*innen haben kann (Jakobs, 2019).

Somit zeigt der aktuelle Stand, dass vor allem in Bezug auf die Integrität und Vertraulichkeit mithilfe von Risikoanalysen entsprechende Maßnahmen, wie eine erweiterte Verschlüsselung der Daten wahrgenommen werden müssen. So muss 
beispielsweise gewährleistet werden, dass bei Implantaten die festgelegte Herzfrequenz beibehalten wird und Dritte nicht an die Signale gelangen dürfen (Jakobs, 2019).

Um die Telemedizin nachhaltig abzusichern, müssen die Grundsätze des Datenschutzes stets verfolgt werden. Diese Aufgabe umfasst eine datenschutzgerechte Organisation der Technik, sowohl auf menschlicher Ebene als auch im allgemeinen Umgang mit Sicherheitslücken und Weiterentwicklungen von Standards und Leitfäden (Staufer, 2019).

\section{Finanzierung}

Bevor ein neues System in der Breite Anwendung findet, muss zuerst der Finanzierungsaspekt geklärt werden. Nachfolgend werden die wichtigsten Fakten zur Finanzierung von Telemonitoring bei Herzinsuffizienz beleuchtet. Dazu zählen die Abrechnung, Kostenerstattung durch Krankenversicherung und die Infrastrukturkosten.

\section{Invasives Telemonitoring}

Seit April 2016 ist die telemedizinische Funktionsanalyse bei implantierbaren Kardioverter Defibrillatoren (ICD) und Systemen zur kardialen Resynchronisationstherapie (CRT) Leistung des Einheitlichen Bewertungsmaßstabes (EBM). Die Abrechnung der EBM-Leistung erfolgt über die zuständige Kassenärztliche Vereinigung durch den behandelnden Arzt. Diese Entscheidung basiert auf dem verbindlichen Beschluss des Erweiterten Bewertungsausschusses nach $\S 87$ Abs. 4 SGB V in seiner 42. Sitzung am 15. Dezember 2015. Damit es den Ärzt*innen möglich ist, eine telemedizinische Funktionsanalyse durchzuführen, wird eine anbieterspezifische Telemedizin-Infrastruktur benötigt, die aus einer gesicherten Datenübertragung, der Bereitstellung der Serverleistung, der Anwender-Software, der Datenaufbereitung, dem Full- Service inkl. Garantie und Geräteaustausch und der Nutzung der Patient*innen- und Arzthotline sowie einer Übertragungs-App- oder einem Monitor für das Implantat besteht. Die Kostenerstattung dieser Infrastruktur, die für die Durchführung der telemedizinischen Funktionsanalyse zwingend erforderlich ist, wird jedoch durch einige gesetzliche Krankenversicherungen nach wie vor abgelehnt.

Abzugrenzen von der geplanten telemedizinischen Funktionsanalyse ist das kontinuierliche Telemonitoring durch kardiale Implantate. Dieses befindet sich aktuell in der Nutzenbewertung durch den GBA. Eine Erstattung der Infrastrukturkosten im Rahmen des kontinuierlichen Telemonitoring erfolgt daher momentan nicht (BVMed e. V., 2018). 


\section{Nichtinvasives Telemonitoring}

Mit dem Inkrafttreten des Digitalen Versorgungs-Gesetzes (DVG) im Jahr 2020 sollen in Zukunft bestimmte digitale Gesundheitsanwendungen, wie beispielsweise Gesundheits- oder Medizin-Apps, für gesetzlich Versicherte zu einer Kassenleistung werden. Die Kostenübernahme erfolgt dann, wenn diese Apps vom Bundesinstitut für Arzneimittel und Medizinprodukte auf Datensicherheit, Datenschutz und Funktionalität geprüft worden sind, den Patient*innen vom behandelnden Arzt verordnet wurden oder als Voraussetzung eine begründete Diagnose vorliegt. Vitalparameter wie Puls, Blutdruck oder das Gewicht werden dabei vom Patienten erfasst und mithilfe der Apps direkt an den Arzt/Ärztin gesendet (Verbraucherzentrale NRW, 2019).

\section{Diskussion}

Die Ergebnisse der Literaturanalyse haben zahlreiche Aspekte in Bezug auf die Anwendung von Telemonitoring in der Kardiologie aufgezeigt. Diese sollen im folgenden Abschnitt differenziert und kritisch beleuchtet werden. Dabei wird die Struktur der einzelnen Kriterien beibehalten.

Im Bereich der technischen Möglichkeiten zeigt sich, dass es sich um ein breites Feld an verschiedenen Technologien handelt, die zum Einsatz kommen können. Nach ihrer Funktionsweise unterschieden, wird eine Klassifizierung anhand drei Generationen vorgenommen. Dies führt dazu, dass die unterschiedlichen Systeme im Hinblick auf weitere Aussagen differenziert betrachtet werden müssen und nicht gleichgestellt werden dürfen. Was sich unter anderem in dem Beispiel der unterschiedlichen Handhabungen gerade im Bereich der Notfallmedizin aber auch im Finanzierungsaspekt zeigt.

Zudem könnte es eine Herausforderung für Ärzt*innen werden, die richtige Generation des Telemonitoring Systems für jeden Betroffenen individuell zu wählen. Da es gravierende Unterschiede in der Möglichkeit zum schnellen Intervenieren im Notfall gibt, ist die Entscheidung mit welchem System aus welcher Generation der Patient überwacht wird mit großer Sorgfalt auszuwählen. Nicht alle Patient*innen mit Herzinsuffizienz können mit Systemen der dritten Generation versorgt werden, da es dafür aktuell zu wenig Fachärzt*innen gibt, die die Kapazitäten haben, kontinuierlich Daten von Patient*innen auszulesen und im Notfall schnell zu handeln. Je mehr verschiedene Möglichkeiten es zum Telemonitoring der Herzinsuffizienz gibt, desto unübersichtlicher wird der Markt und desto mehr Forschung muss betrieben werden, um die Evidenz der verschiedenen Systeme zu belegen und so die Grundlage für einen flächendeckenden Einsatz zu schaffen. Dieser Aspekt kann sowohl Chancen bieten der Individualität der Patient*innen gerecht zu werden, allerdings birgt er auch die Gefahr den Überblick zu verlieren. 
Die Vielfältigkeit der technischen Möglichkeiten stellte in der weiteren Bearbeitung der einzelnen Kriterien eine Erschwernis dar, da möglichst viele Technologien mit abgedeckt werden sollten.

Das Kriterium der Chancen und Risiken für das Gesundheitssystem zeigte, dass Telemonitoring als Ergänzung dienen und keine bestehenden Systeme ersetzen soll. Die Vorteile der Telekardiologie bestehen darin, dass dem demographischen Wandeln und dem zunehmenden Fachärztemangel v. a. in ländlichen Regionen entgegengewirkt werden kann. Ein weiterer Vorteil für das Gesundheitssystem ist die Reduktion der Rehospitalisierungen und die Dauer von Krankenhausaufenthalten. Dies verringert nicht nur die Kosten für das Gesundheitssystem, sondern entlastet es auch, indem es u. a. personelle Ressourcen einspart. Durch den akuten Personalmangel sowohl im stationären als auch im ambulanten Sektor ist die Reduzierung von vermeidbaren Ereignissen durchaus anzustreben. Allerdings wird auch für die Etablierung neuer Versorgungsstrukturen, beispielsweise dem Aufbau von telemedizinischen Zentren, einerseits qualifiziertes Personal benötigt, dass relevante Abweichungen vorher festgelegter Grenzwerte zuverlässig erkennt und den betreuenden Arzt informiert, anderseits wird für Systeme der dritten Generation ärztliches Personal benötigt, dass bei Abweichungen der Daten Kontakt mit dem Patienten aufnimmt und ggf. Notfallmaßnahmen ergreift. Diese Ressourcen müssen erstmal geschaffen werden.

Das primäre Ziel des Telemonitoring sollte nicht der ökonomische Nutzen für das Gesundheitssystem, sondern vor allem eine optimierte Versorgungsstruktur und ein damit einhergehender Gewinn an Lebensqualität für die Nutzergruppe, d. h. in diesem Fall Patient*innen mit einer chronischen Herzinsuffizienz, sein. Wie bereits erwähnt profitieren diese sowohl durch eine verringerte Sterblichkeitsrate als auch durch die Reduzierung der Dauer von Krankenhausaufenthalten was verschiedene Studien belegen. Weitere Aspekte werden in Studien nicht aufgegriffen. Allerdings geht der Nutzen für Patient*innen weit über diese Aspekte hinaus. Telemonitoring hat großes Potenzial, die Versorgungsqualität merklich zu verbessern. Mit der implantatbasierten Fernüberwachung wichtiger Vitalparameter kann die räumliche Distanz zwischen Arzt und Patient überbrückt und die Therapie bedarfsorientiert für den Patienten angepasst werden. Arztbesuche und therapeutische Konsequenzen lassen sich somit individuell an den Patientenzustand anpassen und klinisch sinnvoll planen. Durch einen zunehmenden Mangel an Fachärzt*innen, vor allem in ländlichen Regionen, entsteht ein wachsendes Versorgungsdefizit, was durch Telekardiologie teilweise kompensiert werden könnte. Außerdem werden die entsprechenden Werte zeitnah von Fachpersonal oder Programmen überwacht, was die Fehlinterpretation von Laien verhindert. Nicht nur akute Notsituationen werden somit frühzeitig erkannt, auch Verschlechterungen werden registriert und es kann 
zeitnah interveniert werden. Wie die Grafik der Diskrepanz zwischen der subjektiven Wahrnehmung und der objektiven Verschlechterung des Gesundheitszustandes im Ergebnisteil veranschaulicht, kann mit geeigneter telemedizinischer Betreuung eine beginnende objektive Verschlechterung diagnostiziert werden, bevor der Patient sie wahrnimmt. Die kann als Chance für ein besseres Outcome der Patient*innen gesehen werden und zu einem verstärkten Sicherheitsgefühl führen. Eben genannte Aspekte sind aktuell noch nicht Gegenstand der Forschung, da die Systeme hierfür flächendeckend und über einen längeren Zeitraum eingesetzt werden müssten, was aufgrund verschiedenster Faktoren noch nicht gegeben ist. Zudem sind Faktoren wie das Sicherheitsgefühl schwer zu erfassen da die Systeme im Rahmen des Alltags und nicht im Rahmen einer Studie mit ohnehin engerer Überwachung zum Einsatz kommen.

Vor allem im Bereich der Notfallmedizin gibt es Systeme, welche Patient*innen im akuten Notfall helfen können. Sie werden optimaler Weise dort eingesetzt, wo schneller Handlungsbedarf erforderlich ist. Den in den Ergebnissen bereits aufgeführten Systemen ist es möglich, akut lebensbedrohliche Situationen zu erfassen. Dies ist insbesondere dann bedeutsam, wenn der*die Patient*in allein Zuhause ist und sonst keiner den Notfall erkennen würde. Die Literaturrecherche zeigte hier erneut, dass es eine Vielzahl an Systemen gibt, die allerdings nicht zum Einsatz kommen, da sie sich überwiegend in der Testphase befinden. Zudem ist erkennbar, dass es noch keine einheitliche Auswertung der Daten gibt, da entweder Ärzt*innen und Gesundheits- und Krankenpfleger*innen in der Klinik bzw. nichtärztliches Personal in sogenannten Servicecentern dieser Aufgabe nachkommt oder die Auswertung anhand elektronischer Systeme erfolgt. Auch das Vorgehen bei Abweichungen von den Standardwerten ist noch nicht einheitlich geregelt. Die Handlungsmöglichkeiten reichen vom Informieren eines Facharztes, der sich mit dem Patienten in Verbindung setzt, bis hin zur automatischen Alarmierung des Rettungsdienstes. Es müssten flächendeckende Strukturen geschaffen und ein einheitlicher Handlungsplan festgelegt werden. Der Mangel an Literatur ist ein weiteres Indiz dafür, dass Telemonitoring gerade im Bereich der Notfallmedizin noch stark ausbaufähig ist. Im Bereich der Notfallmedizin geht es um akute Ereignisse, welche i. d. R. einen schnellen Handlungsbedarf erfordern. Mithilfe der in der Analyse beschriebenen Systeme ist es möglich Notfallsituationen zu erfassen, auch wenn der*die Patient*in beispielsweise allein zuhause ist und keiner den Notfall erkennen würde.

Grundlage für die Etablierung von Telemonitoring Systemen ist unter anderem die sichere Verarbeitung der Daten. Die neuen gesetzlichen Regularien wie das IT-Sicherheits- und E-Health- Gesetz, sowie die europäische Datenschutzgrundverordnung zeigen, wie wichtig diese Thematik für das Gesundheitssystem ist. 
Die Literaturrecherche belegte, dass das Thema Datenschutz im Bereich Telekardiologie äußerst relevant ist. Da es sich um sehr sensible Daten handelt, muss gewährleistet sein, dass diese sicher übermittelt werden können. Allerdings ist dies aktuell noch nicht in angemessenem Umfang möglich, wie negativ Beispiele zeigen und bedarf weiterer politischer Handlungen. Ein Patientenschutzgesetz soll eingeführt werden, um ein Vorgehen mit bestehenden Sicherheitslücken zu regeln. Der Zugriff Unbefugter bzw. die Manipulation von Daten können fatale Folgen für die Gesundheit der Patient*innen haben. Es wäre fahrlässig, Telemonitoring für eine breite Masse, ohne einen weitreichenden Schutz einzuführen, der die Daten, technischen Eistellungen und Funkwellen vor Hackerangriffen sicher macht. Durch die Digitalisierung im Gesundheitssystem ist somit eine Anpassung des Sicherheitsmanagements unumgänglich. Solange dies nicht in ausreichendem Maße gegeben ist, ist die flächendeckende Anwendung der Telemonitoring Systeme im Gesundheitssystem als eher unwahrscheinlich und risikobehaftet einzuschätzen.

Aus der bisherigen Analyse ist ein Nutzen der Telekardiologie erkennbar. Allerdings muss beim Thema Telemonitoring auch die Finanzierung betrachtet werden. Nur Systeme, bei denen der Finanzierungsaspekt im Vorfeld geklärt ist, werden flächendeckend Einsatz finden. Das Kriterium der Finanzierung geht aus der Analyse als ein weiteres Hindernis für die Etablierung von Telemonitoring Systemen hervor. Zwar wurde Telekardiologie vor einigen Jahren bereits in die europäischen Behandlungsleitlinien aufgenommen, jedoch ist die Kostenübernahmesituation in Deutschland weiterhin unbefriedigend. Bis heute ist die nicht vorhandene Regelvergütung des kontinuierlichen Telemonitoring für Implantat-Patient*innen das größte Hindernis für dessen breite Anwendung. Kontinuierliches Telemonitoring befindet sich seit mehr als 3 Jahren in der Nutzenbewertung durch den GBA womit begründet wird, dass die Erstattung der Infrastrukturkosten momentan nicht erfolgt.

Laut Studienlage ist es eindeutig, dass ausreichend klinische Evidenz zu den Vorteilen des Telemonitoring von aktiven kardiologischen Implantaten vorliegt. In mehreren randomisierten Untersuchungen konnte gezeigt werden, dass durch konsequente telemedizinische Begleitung die Zeit bis zur Wahrnehmung technischer und klinischer Probleme relevant verkürzt wird und Krankenhausaufenthalte verhindert werden konnten. Hier stellt sich die Frage, warum der GBA sich so lange mit der Nutzenbewertung aufhält, da diese Grundvoraussetzung für die Finanzierung ist.

Die telemedizinische Funktionsanalyse wird bereits als Leistung des Einheitlichen Bewertungsmaßstabes übernommen, jedoch wird die Infrastruktur, die dafür zwingend notwendig ist, nicht von allen Kassen finanziert. Daher findet sie trotz einiger Studien, die eine Evidenz der verschiedenen Systeme belegen aufgrund 
ungeklärter finanzieller Aspekte keine Regelanwendung. Folglich wird es weiterhin nur Pilotprojekte einzelner Krankenkassen geben und die Mehrheit der Patient*innen mit Herzinsuffizienz wird weiterhin keinen Zugriff darauf haben, solange finanzielle Aspekte nicht klar geregelt sind. Als Chance könnte das Inkrafttreten des Digitalen Versorgungs- Gesetzes (DVG) im Jahr 2020 gesehen werden. Kosten für beispielsweise Apps, die zur Übermittlung von Telemonitoring Daten genutzt werden können, werden nach dem Prüfen auf Datensicherheit, Datenschutz und Funktionalität als Kassenleistung übernommen. Vitalparameter wie Puls, Blutdruck oder das Gewicht werden dabei von Patient*innen erfasst und mithilfe der Apps direkt an den Arzt gesendet. Dies löst noch nicht das Problem der vollständigen Finanzierung der Infrastruktur der Funktionsanalyse, ist jedoch ein Schritt in Richtung Teilfinanzierung des Telemonitoring.

\section{Fazit}

Durch die aus der Literaturanalyse resultierenden Kriterien wurde den Autor*innen vor allem die Weitläufigkeit der Thematik ersichtlich. Es zeigte sich im Rahmen der Literaturrecherche, dass viele Artikel, Studien, sowie Metaanalysen zu Telemonitoring in der Kardiologie vorhanden sind. Dabei war auffällig, dass diese teilweise schon älter sind. Daher ist es erstaunlich, dass.

Telekardiologie außerhalb der Forschung im Alltag kaum bis gar nicht angewendet wird, obwohl ein Nutzen sowohl für die Patient*innen als auch für das Gesundheitssystem als bewiesen gilt. Telemonitoring hat großes Potenzial die Versorgungsqualität merklich zu verbessern. Gerade im Hinblick auf diverse Versorgungsdefizite, welche weiter zunehmen werden, bietet das Telemonitoring die Chance, das ohnehin schon oft überlastete Personal des Gesundheitssystems zu entlasten, indem es Möglichkeiten zur Überwachen auch von Zuhause aus schafft. Die Daten können in der individuellen Versorgung genutzt werden und tragen damit zur längerfristigen Verbesserung der Versorgungssituation bei. Für Patient*innen mit Herzinsuffizienz erhöhen die Telemonitoring Systeme das Sicherheitsgefühl, da ihr Gesundheitszustand auch außerhalb der Klinik überwacht wird und im Notfall schnell gehandelt werden kann. Ein weiterer wichtiger Aspekt ist die Stärkung der Handlungsfähigkeit der Betroffenen, denn sie können anhand der Daten Belastungssituationen erkennen und eigenständig regulieren. Dies trägt nicht nur zu mehr Autonomie in der Krankheitsbewältigung, sondern auch zur längerfristigen Verbesserung der Versorgungssituation bei. Ein systemrelevanter Veränderungsbedarf ist dahingehend notwendig, dass Aspekte zum Datenschutz und zur Finanzierung geklärt werden müssen, bevor Telemonitoring flächendeckend eingesetzt werden kann, da diese beiden Kriterien nach wie vor ein Hindernis darstellen. Der 
Datenschutz ist ohnehin ein Thema, welches mit der bereits laufenden Digitalisierung im Gesundheitswesen in den nächsten Jahren bearbeitet werden muss. Letztendlich sollte die Möglichkeit zur Verbesserung der Lebensqualität mit dem Einsatz von Telemonitoring bei Patient*innen mit Herzinsuffizienz aus Sicht der Autor*innen Grund genug sein, die bereits bestehenden Technologien auszubauen und flächendecken einzusetzen.

\subsection{Analyse der Prognose von Technologieentwicklungen auf Grundlage des Gartner Hype Cycles for Emerging Technologies}

Vorwort des Betreuers: Jürgen Seitz

Ambient Assisted Living (AAL) gewinnt an Bedeutung. Dies zeigt sich an einer zunehmenden Zahl von Projekten und Veröffentlichungen. Erstaunlicherweise taucht der Begriff bislang nicht in Gartner's Hype Cycle of Emerging Technologies auf. Es tauchen lediglich Begriffe verwandter Gebiete (z. B. Mobile Health Monitoring) oder Themen (z. B. Connected Home), die auch im Kontext von AAL eine Rolle spielen, auf.

Der folgende Beitrag untersucht nicht, weshalb AAL in Gartner's Hype Cycles of Emerging Technologies in den letzten fünfzehn Jahren keine Rolle spielt, sondern es wird grundsätzlicher untersucht, inwiefern die Zielgruppe von Gartner's Veröffentlichungen auf der Grundlage des Hype Cycle of Emerging Technologies strategische Entscheidungen treffen kann. Hierzu wird bspw. untersucht, ob Begriffe über einen längeren Zeitraum auftauchen und ob es Inkonsistenzen bezüglich der Vorhersagezeiträume gibt.

Benjamin Dorn, Julian Germani, Michael Geywitz, Manuel Remlinger, und Felix Schüßler.

\section{Einführung}

In der heutigen Zeit sind Unternehmen, Universitäten und Hochschulen, genauso wie Forschungseinrichtungen und die Politik gezwungen, bedeutsame Zukunftstechnologien frühzeitig zu erkennen, um Maßnahmen einleiten zu können (Kurz, 2016; Fritsch et al., 2008, S. 5; Schleiter, 2014; Braun et al., 2013, S. 15).

Ein mögliches Hilfsmittel dazu ist der Gartner Hype Cycle for Emerging Technologies (van Lente et al., 2013, S. 1615). Dieses ist notwendig, da Prognosen für Technologien, die zu-künftig relevant sein werden, komplex und aufwendig zu erstellen sind (Braun et al., 2013, S. 15). Dies kann dazu führen, dass Technologien 
falsch eingeschätzt werden. Solche Fehleinschätzungen können in verschiedenen Ausprägungen vorkommen. Wurde sich beispielsweise auf eine fehlerhafte Prognose verlassen, kann dies zu strategischen Fehlentscheidungen oder falschen Forschungsschwerpunkten führen (Stumpf \& Lorenzen, 2017). Eine Möglichkeit, um solche Risiken besser abschätzen zu können, ist die folgende Analyse, die die Genauigkeit des Gartner Hype Cycles for Emerging Technologies untersucht. Hierzu werden die Gartner Hype Cycles for Emerging Technologies der Jahre 2005 bis 2019 analysiert.

Das Ziel der Arbeit ist zu erkennen, welchen Beitrag zur Entscheidungsfindung die Gartner Hype Cycles for Emerging Technologies leisten können. Dies geschieht durch die Beantwortung der folgenden Fragestellung: Welche Genauigkeit und welche Aussagekraft besitzt der Gartner Hype Cycle for Emerging Technologies hinsichtlich der Prognose der zeitlichen und existenziellen Verfügbarkeit zukünftiger Technologien?

Zunächst erfolgt die Charakterisierung des Gartner Hype Cycles for Emerging Technologies, um ein grundlegendes Verständnis für die Thematik zu schaffen. Anschließend wird untersucht, ob es bereits anerkannte und publizierte wissenschaftliche Abhandlungen gibt, die sich mit derselben oder einer ähnlichen Problemstellung befassen. Ziel dieser Untersuchung ist es, den Vergleich der Ergebnisse der hier durchgeführten Analyse mit den Resultaten anderer zu ermöglichen. Anschließend wird die Planung der durchzuführenden Analyse beschrieben. Zum einen wird definiert, welche Hype Cycles und welche Technologien Gegenstand der Analyse sind und welche Informationen ausgewertet werden. Zum anderen werden die Kriterien festgelegt, anhand derer die Hype Cycles untersucht werden.

Dabei wird aufgezeigt, wie die relevanten Daten aus den Hype Cycles extrahiert und für die Analyse aufbereitet werden. Anschließend wird dargestellt, wie die Daten konkret ausgewertet werden und welche Ergebnisse aus der Analyse hervorgehen. Zum Abschluss werden die ermittelten Ergebnisse diskutiert. Am Ende werden die wesentlichen Erkenntnisse der Arbeit zusammengefasst und die erarbeiteten Ergebnisse reflektiert. Abschließend erfolgt ein Ausblick in die Zukunft.

\section{Gartner Hype Cycle for Emerging Technologies}

Im folgenden Kapitel werden die wesentlichen Charakteristika des Hype Cycles vorgestellt. Anschließend werden die Ergebnisse bereits publizierter wissenschaftlicher Abhandlungen dargestellt. 


\section{Charakterisierung}

Gartner ist ein weltweit tätiges Forschungs- und Beratungsunternehmen, das Marktforschungsergebnisse und Analysen über die Entwicklungen von Trends anbietet (Gartner Inc., 2020). Unter anderen veröffentlicht Gartner jährlich den Hype Cycle for Emerging Technologies, der neu aufkommende Technologien analysiert (Dedehayir \& Steinert, 2016, S. 29; Rotolo et al., 2015, S. 1828).

Der Hype Cycle for Emerging Technologies ist eine grafische Darstellung des Technologielebenszyklus, der die Reife und Akzeptanz von neuen Technologien prognostiziert. Dadurch erhalten Unternehmen einen Überblick über den Entwicklungsstand von neuen und noch nicht etablierten Technologien zu einem bestimmten Zeitpunkt. Der Hype Cycle bietet Entscheidungsträgern eine Hilfestellung zur selbstständigen Bewertung und Einschätzung des Potenzials und der Relevanz eines Technologietrends (Dedehayir \& Steinert, 2016, S. 29). Innerhalb des in Abb. 5.6 dargestellten Gartner Hype Cycles werden auf der Y-Achse die Erwartungen an eine Technologie abgebildet, während die X-Achse die Phasen beschreibt.

Gartner unterteilt, aufgrund der Erwartungen und der produktiven Einsatzmöglichkeiten einer Technologie, die Zeit in fünf verschiedene Phasen. Diese geben

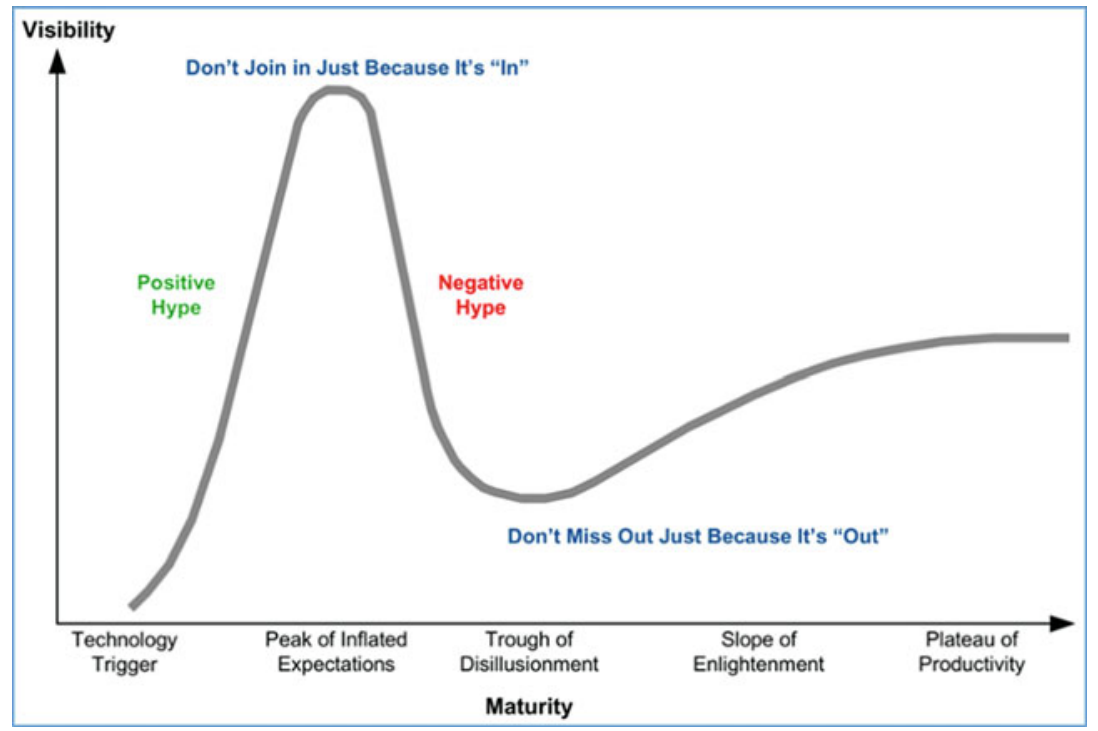

Abb. 5.6 The Gartner Hype Cycle (Linden \& Fenn, 2003, S. 5) 
Aufschluss über den Entwicklungsstand einer Technologie (Kreutzer 2015, S. 3). Im Folgenden werden die Phasen des Hype Cycles dargestellt:

1. Technology Trigger („Technologischer Auslöser“).

Ein neuer, potenzieller, technischer Durchbruch beginnt sich durch erste öffentliche Ankündigungen, Präsentationen oder Pressemitteilungen zu verbreiten. Die dadurch entstandene Aufmerksamkeit weckt erstes Interesse der Industrie an der neuen Technologie. Ob die Technologie sich durchsetzt, ist zu diesem frühen Stadium noch nicht absehbar (Linden \& Fenn, 2003, S. 7; Kreutzer, 2015, S. 3).

2. Peak of Inflated Expectations (,Höhepunkt der überzogenen Erwartungen“).

Diese Phase ist durch den Enthusiasmus und die unrealistischen Erwartungen an die neue Technologie gekennzeichnet, die zusätzlich von den Medien noch verstärkt werden. Zunehmend beginnen Unternehmen zu prüfen, ob die Technologie für ihre Geschäftsstrategie relevant ist. Allerdings sind die Investitionsmöglichkeiten in dieser Phase risikobehaftet (Linden \& Fenn, 2003, S. 8; Kreutzer, 2015, S. 3).

3. Trough of Disillusionment (,Tal der Enttäuschungen“).

Die Technologie wird den unrealistischen und überzogenen Erwartungen der Unternehmen und Medien nicht gerecht. Die Anzahl der gescheiterten Projekte steigt, wodurch das Interesse der Unternehmen und Medien schwindet. Einige Unternehmen finden inmitten der Desillusionierung Lösungsansätze, die durch die frühen Rückmeldungen zu den Problemen und Fragen entstanden und erkennen den Mehrwert der Technologie (Linden \& Fenn, 2003, S. 8; Kreutzer, 2015, S. 4).

4. Slope of Enlightenment (,Pfad der Erleuchtung“).

Gezielte Experimente und praktische Erfahrungen führen zu einem besseren Verständnis der neuen Technologie. Dieses Verständnis umfasst die Anwendbarkeit, die Risiken und Grenzen sowie den Nutzen der Technologie. Einige Unternehmen setzen weiter auf die Technologie und bringen Produkte der zweiten und dritten Generation auf den Markt (Linden \& Fenn, 2003, S. 8; Kreutzer, 2015, S. 4).

5. Plateau of Productivity (,Plateau der Produktivität“).

Die letzte Phase stellt den Beginn der allgemeinen Einführung der Technologie dar. Die endgültige Höhe des Plateaus variiert, je nachdem, ob die Technologie allgemein anwendbar ist oder nur einem Nischenmarkt zugutekommt (Linden \& Fenn, 2003, S. 8; Kreutzer, 2015, S. 4).

Die Dauer, die Technologien benötigen, um alle Phasen des Hype Cycles zu durchlaufen, unterscheidet sich. Gartner gibt nicht nur den Entwicklungsstand zu einer 
Technologie wieder, sondern auch eine Prognose, wie lange eine Technologie benötigt, um das Plateau zu erreichen. Hierbei verwendet Gartner zum einen die vier Prognosezeiträume: kleiner 2 Jahre, 2-5 Jahre, 5-10 Jahre und größer 10 Jahre. Um vorherzusagen, wie lange eine Technologie bis zum Erreichen des Plateaus benötigt, verwendet Gartner zum anderen den Prognosewert ,,veraltet vor dem Erreichen des Plateaus“, um die Technologie frühzeitig aus dem Hype Cycle zu entfernen (Dedehayir \& Steinert, 2016, S. 29).

\section{Kritische Analysen der bisherigen Forschung}

Der Hype Cycle for Emerging Technologies wurde in früheren wissenschaftlichen, empirischen Arbeiten bereits untersucht. Es wurde festgestellt, dass dieser Unstimmigkeiten aufweist (Dedehayir \& Steinert, 2016, S. 29). Dazu wurde ein Schema zur tabellarischen Darstellung der Daten entwickelt. In diesem wird ein Zeitraum von 2003 bis 2009 untersucht (Dedehayir \& Steinert, 2016, S. 35). Dies scheint für einen seit 1995 bestehenden Hype Cycle ein kurzer Zeitraum zu sein (Dedehayir \& Steinert, 2016, S. 29). Außerdem stellt sich die Frage, warum im Jahre 2016 lediglich ein Zeitraum bis 2009 untersucht wurde, statt einen Bezug zu aktuelleren Technologien herzustellen.

Das Ergebnis dieser Veröffentlichung ist, dass es Unstimmigkeiten bei dem von Gartner aufgestellten Hype Cycle for Emerging Technologies gibt. Daher ist es erforderlich, eine Publikation zu schaffen, die zusätzlich aktuellere Technologien untersucht.

\section{Planung}

Im folgenden Kapitel wird beschrieben, welche Einschränkungen und Kriterien zur Durchführung der Analyse aufgestellt werden. Diese sind zur Eingrenzung der Analyse notwendig.

Der Gartner Hype Cycle for Emerging Technologies wird seit 1995 jährlich veröffentlicht (Dedehayir \& Steinert 2016, S. 29). Die folgende Analyse konzentriert sich auf die letzten 15 Jahre des Hype Cycles. Es werden einschränkend die Jahre 2005 bis 2019 betrachtet. Die Analyse der Hype Cycles erfolgt anhand von quantitativen und qualitativen Kriterien. An dieser Stelle werden zunächst die quantitativen Kriterien betrachtet. Zur Umsetzung dieses Vorgehens wird eine Datengrundlage benötigt. Diese Datengrundlage wird aus der von Gartner frei zugänglich bereitgestellten graphischen Darstellung des Hype Cycles bezogen und anschließend automatisiert ausgewertet (Gartner Inc., 2005, 2006, 2007,2008, 2009, 2010, 2011, 2012, 2013, 2014, 2015, 2016, 2017, 2018, 2019; Dawson, 2017, S. 2; IDG Business Media GmbH, 2005, 2006, 2007, 2008, 2009, 2010, 2011, 2012, 2013). Bevor die Untersuchung anhand der quantitativen Kriterien erfolgen kann, müssen die Daten 
vorbereitet werden. Hierzu wird geprüft, ob eine Technologie mindestens zweimal im untersuchten Zeitraum aufzufinden ist. Ist dies nicht der Fall, wird sie aussortiert. Dies hat den Hintergrund, dass die quantitativen Kriterien nicht untersucht werden können, wenn eine Technologie nur einmal vorkommt, da kein Vergleich zwischen zwei Prognosen zur gleichen Technologie möglich ist. Die Frage, weshalb eine Technologie nur einmal auftaucht, ist nicht weniger interessant. Diese Frage wird jedoch im Rahmen dieser Arbeit nicht weiterverfolgt.

Die quantitativen Kriterien sind „Vorkommen“, „Korrekter Phasenverlauf“ und „Korrekte Prognose“. Abb. 5.7 zeigt das Vorgehensschema, nach dem diese Kriterien geprüft werden.

Das Kriterium Vorkommen beschreibt das konstante Auftreten einer Technologie über mehrere Jahre. Mithilfe dieses Kriteriums soll die Forschungsfrage bezüglich der existenziellen Genauigkeit beantwortet werden. Dabei wird das Kriterium als richtig klassifiziert, wenn die Technologie vom ersten Auftreten im Hype Cycle bis zum Verschwinden aus dem Hype Cycle jährlich auftritt. Wenn eine Technologie von 2010 bis 2015 den Hype Cycle durchläuft, muss sie jährlich neu prognostiziert werden. Das Kriterium korrekter Phasenverlauf beschreibt, ob die Technologien die Phasen in der richtigen Reihenfolge durchlaufen oder ob sie rückläufig sind. So wird beispielsweise ein Verlauf von Phase 1 in Phase 2 genauso korrekt angesehen, wie das Verbleiben in Phase 1 Dies begründet sich zum einen dadurch, dass eine Technologie den kompletten Phasenverlauf nehmen soll, ihn aber nicht nehmen muss.

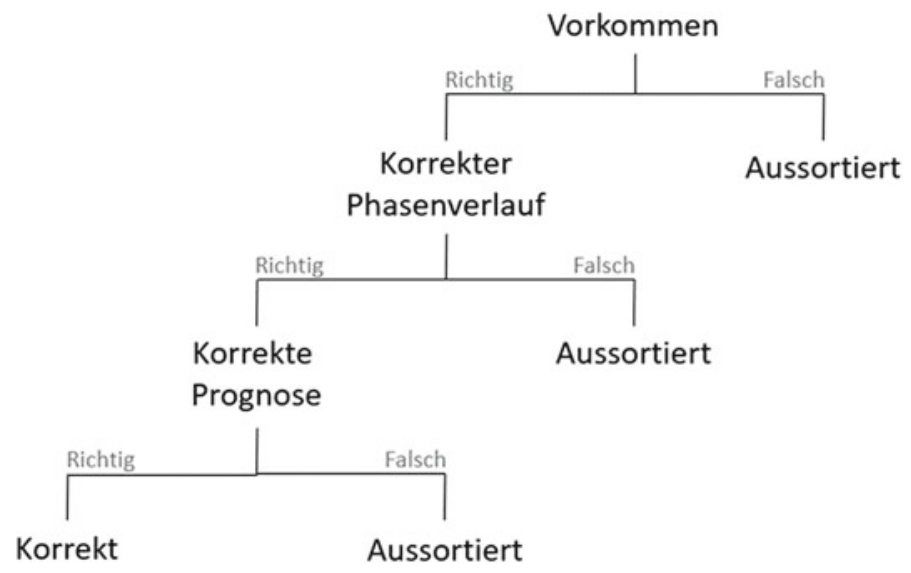

Abb. 5.7 Vorgehensschema 
Zum anderen besitzt jede Technologie eine eigene Entwicklungsgeschwindigkeit. Hingegen als falsch klassifiziert wird eine Technologie, die von Phase 3 - Trough of Disillusionment - zurück in Phase 2 - Peak of Inflated Expectations - wechselt, da die in Phase 3 beschriebene Enttäuschung erst durch die in Phase 2 erzeugten überzogenen Erwartungen entstehen kann. Der Trend muss nicht zwangsläufig in Phase 1 entstehen und kann Phasen überspringen. Daher wird nur eine Rückläufigkeit bei den Phasen als falsch angesehen. Es ist im Einzelfall zu untersuchen, weshalb beispielsweise eine Technologie Phase 1 überspringt und das erste Mal gleich in Phase 2 im Hype Cycle erscheint. Das letzte quantitative Kriterium ist die korrekte Prognose. Hierbei wird untersucht, ob die von Gartner aufgestellte Prognose plausibel ist. Mit falsch bewertet wird dieses Kriterium, wenn die Prognose nach oben oder in einem Jahr um zwei Prognosestufen nach unten korrigiert wird. Beispielsweise wird ein Trend als falsch eingeordnet, wenn 2015 die Prognose 5-10 Jahre bis zum Erreichen des Plateaus ist und 2016 dann $<2$ Jahre, da die Stufe 2-5 Jahre übersprungen wurde. Die beiden zuletzt beschriebenen Kriterien dienen dazu, die Forschungsfrage im Hinblick auf die zeitliche Genauigkeit zu beantworten.

Anhand der qualitativen Kriterien wird untersucht, ob formale Inkonsistenzen in den Hype Cycles auftreten. In Abschn. 4.3 werden die Gartner Hype Cycles bezüglich des Aufbaus und der Einheitlichkeit analysiert. Konkret diskutiert werden dabei die Einheitlichkeit der Technologiebezeichnungen und die formalen Aspekte der Graphen.

Dies wird in im folgenden Kapitel genauer beschrieben.

\section{Durchführung der Analyse}

Im folgenden Kapitel wird zunächst auf die Umsetzung des geplanten Vorgehens eingegangen. Dabei werden sowohl die Erstellung als auch die automatisierte Auswertung beschrieben. Anschließend werden die Ergebnisse dargestellt und diskutiert.

Erstellung des Datasets

In diesem Kapitel wird beschrieben, wie die Extraktion und Aufbereitung der Daten aus den Gartner Hype Cycles erfolgte. Abb. 5.8 zeigt die Vorgehensweise zur Erstellung des Datasets.

Zunächst wurden in einer Excel-Tabelle händisch alle Technologien erfasst, die in den 15 Hype Cycles vorkommen. Für jede Technologie wurde bei jedem Hype Cycle erfasst, ob die Technologie vorkommt, in welcher Phase sie sich befindet und wie die Prognose für das Erreichen des Plateaus ausfällt. Technologien, deren Bezeichnungen ähnlich oder gleich sind und denselben Trend bzw. dieselbe Technologie darstellen, wurden zu einem Begriff zusammengeführt. So wurde beispielsweise die Abkürzung „SOA“ anstelle der Begrifflichkeit „Service Oriented 
Abb. 5.8 Vorgehen zur

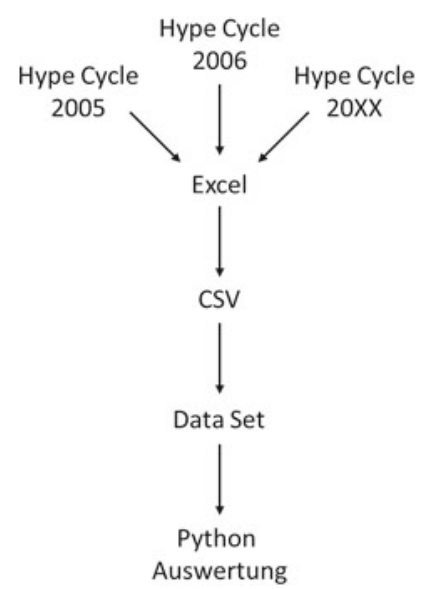

Architecture (SOA)“ verwendet. Im Anhang befindet sich eine Aufstellung aller zusammengeführter Begriffe der unterschiedlichen Technologien.

Im nächsten Schritt wurde die Excel-Tabelle in eine CSV-Datei umgewandelt, damit sie als Dataset für die webbasierte Datenanalyseplattform ,www.kaggle.com“ verwendet werden kann. Tab. 5.2 zeigt die Struktur des ursprünglichen Datasets, das aus der CSV-Datei entstanden ist. Für die Spalten ,,Vorkommen“, „Prognose“ und „Phase“ ist die tatsächliche Struktur des Datasets in verkürzter Form dargestellt. Der Platzhalter ,yy“ steht jeweils für die Kurzform der Hype-Cycle-Jahrgänge von „05“ für das Jahr 2005 bis „,19“ für das Jahr 2019.

Für die Auswertung der in der Excel-Matrix erfassten Daten wurden Routinen zur automatisierten Auswertung mithilfe der Programmiersprache Python entwickelt. Dies hat den Vorteil, dass die Auswertung beliebig oft ausführbar ist, beispielsweise wenn sich an der grundsätzlichen Datenerfassung innerhalb der Excel-Datei etwas ändert. Bei korrekter Abbildung der Kriterien kann zudem die Gefahr manueller Auswertungsfehler ausgeschlossen, beziehungsweise minimiert werden. Der dritte

Tab.5.2 Struktur des ursprünglichen Datasets

\begin{tabular}{l|l}
\hline Spaltenbezeichnung & Datentyp \\
\hline Technologie & String \\
\hline Vorkommen $y y$ & Boolean \\
\hline Prognose $y y$ & String \\
\hline Phase $y y$ & Integer \\
\hline
\end{tabular}


Vorteil, der für die automatisierte Auswertung spricht, ist, dass die Auswertung auch bei Vergrößerung des Datenbestandes schnell möglich ist, wodurch zukünftig auch größere Zeiträume des Hype Cycles teilautomatisiert ausgewertet werden können.

Innerhalb des Programms wird zunächst die erstellte Matrix, die aus der CSVDatei importiert werden kann, in ein anderes Schema konvertiert (siehe Link zum Data Set im Anhang). Jeder Datensatz innerhalb des Datenbestands kann durch die Kombination aus Technologie und Jahr eindeutig identifiziert werden. Abb. 5.9 stellt exemplarisch das Kriterium Vorkommen dar. In diesem Beispiel wird die Feststellung ob eine Technologie ,,vorkommt - nicht vorkommt“ überprüft.

Ergebnisse wurden erzielt, indem jedes der Kriterien gemäß des Vorgehensschemas auf den transformierten Datenbestand angewendet wurde.

\section{Auswertung der Ergebnisse}

Durch die Auswertung der 122 Technologien, die mindestens zweimal im Hype Cycle vorkommen, ergab sich folgendes Ergebnis (Tab. 5.3):

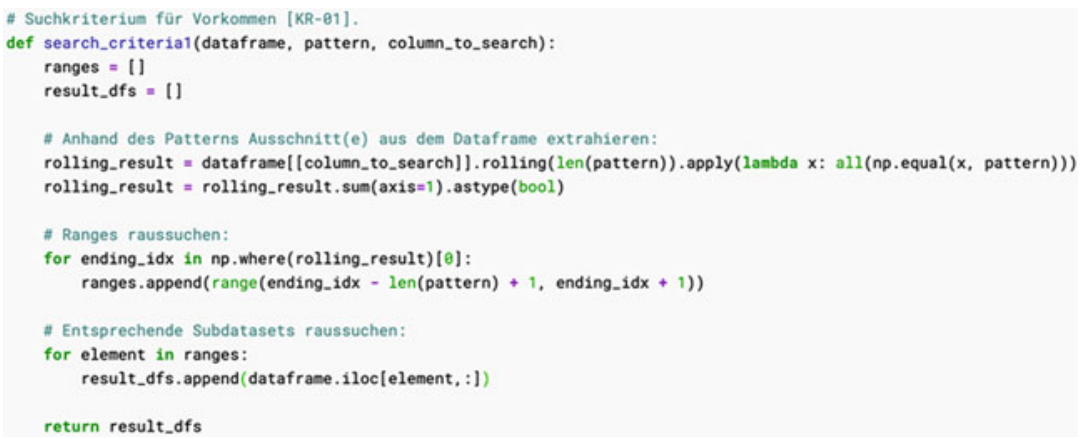

Abb.5.9 Umsetzung des Kriteriums Vorkommen in der Programmiersprache Python

Tab.5.3 Tabellarische Darstellung der Ergebnisse

\begin{tabular}{l|l|l}
\hline & Richtig & Falsch \\
\hline Vorkommen & 97 & 27 \\
\hline Korrekter Phasenverlauf & 90 & 5 \\
\hline Korrekte Prognose & 81 & 9 \\
\hline Gesamt & $\mathbf{8 1}(\mathbf{6 6 , 4} \%)$ & $\mathbf{4 1}(\mathbf{3 3 , 6} \%)$ \\
\hline
\end{tabular}


Bei 33,6 \% der von Gartner untersuchten Technologien liegt daher mindestens ein Kriterium vor, durch welches die Prognose einer Technologie angezweifelt werden kann.

Mithilfe des ersten Kriteriums wird das Vorkommen einer Technologie über einen Zeitraum analysiert. Kommt eine Technologie nicht kontinuierlich im Hype Cycle vor, so wird sie als fehlerhaft eingeordnet. Ein Beispiel für eine fehlerhafte Technologie stellt das „Quantum Computing“ in Abb. 5.5 dar.

Der Graph (Abb. 5.10) stellt das Vorkommen der Technologie von 2005 bis 2019 dar. Auf der X-Achse werden die Jahre abgebildet, während auf der Y-Achse das Vorkommen abzulesen ist. Das Vorkommen kann dabei zwei Zustände einnehmen. Hat das Vorkommen den Wert „0“, bedeutet das, dass die Technologie in diesem Jahr nicht im Hype Cycle aufgetreten ist. Ist der Wert des Vorkommens jedoch „1“, bedeutet das, dass die Technologie in diesem Jahr im Hype Cycle prognostiziert wurde. Die Technologie kommt seit Beginn des Betrachtungszeitraums im Hype Cycle vor. Jedoch wird das Quantum Computing in drei Jahren, nämlich 2007, 2008 und 2010, nicht neu prognostiziert. Von 2011 bis 2018 kommt das Quantum Computing wieder jährlich im Hype Cycle vor. Aus diesen Beobachtungen resultiert die Frage, ob das Ausschließen dieser Technologie aus den Hype Cycles 2007, 2008 und 2010 sinnvoll war. Denn diese Schwankungen innerhalb des Vorkommens können Unternehmen dabei verunsichern, sich im Bereich dieser Technologien zu etablieren. Die Nachvollziehbarkeit der Technologieentwicklung leidet unter dem inkonsistenten Vorkommen.

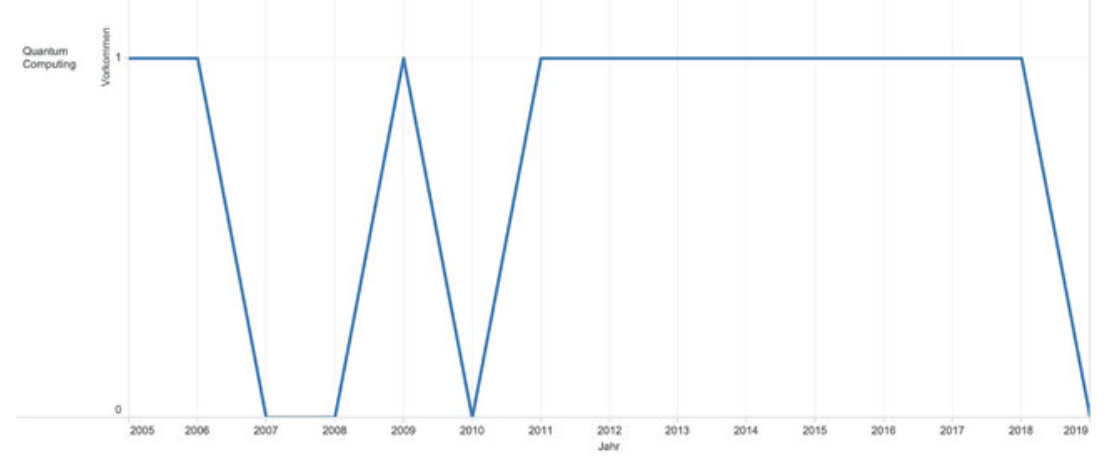

Abb. 5.10 Vorkommen Quantum Computing 
Anhand des zweiten Kriteriums wird der Phasenverlauf einer Technologie analysiert. Verläuft eine Technologie entgegen der Phasenrichtung, beispielsweise von Phase 2 zurück in Phase 1, so wird die Technologie als fehlerhaft kategorisiert. Anhand der in Abb. 5.11 dargestellten Technologie „Internet TV“ wird beispielhaft die Funktionsweise des zweiten Kriteriums erklärt.

Der Graph 5.11 stellt die Entwicklung der Phasen von 2005 bis 2019 dar. Die X-Achse bildet die Jahre ab, während die Y-Achse die fünf Phasen des Hype Cycles darstellt. Die Technologie des Internet TV tritt zum ersten Mal im Jahr 2009 auf und wird von Gartner direkt in die zweite Phase, dem Peak of Inflated Expectations, eingeordnet. Die erste Einordnung ist durchaus plausibel, obwohl die Technologie nicht in der ersten Phase des Hype Cycles beginnt. Das liegt daran, dass Technologien schnell einen starken Hype erleben können und somit innerhalb eines Jahres die gesamte erste Phase des Hype Cycles durchlaufen und direkt in die zweite Phase eingeordnet werden. Jedoch korrigiert Gartner seine Einordnung im Jahr 2010, indem die Technologie zurück in die erste Phase des Hype Cycles gesetzt wird. In den Jahren 2011 und 2012 ordnet sich die Technologie immer eine Phase höher ein, sodass sie im Jahr 2012 die Phase Trough of Disillusionment erreicht. Ab dem Jahr 2013 verschwindet diese Technologie aus dem Hype Cycle und wird bis 2019 auch nicht wieder aufgenommen. Die Phasenentwicklung dieser Technologie wirft beim Betrachter die Frage nach der Korrektheit der Phaseneinordnung in den Jahren 2009 und 2010 auf. Tatsächlich empfangen in Deutschland 3,1 Mio. Haushalte Internet

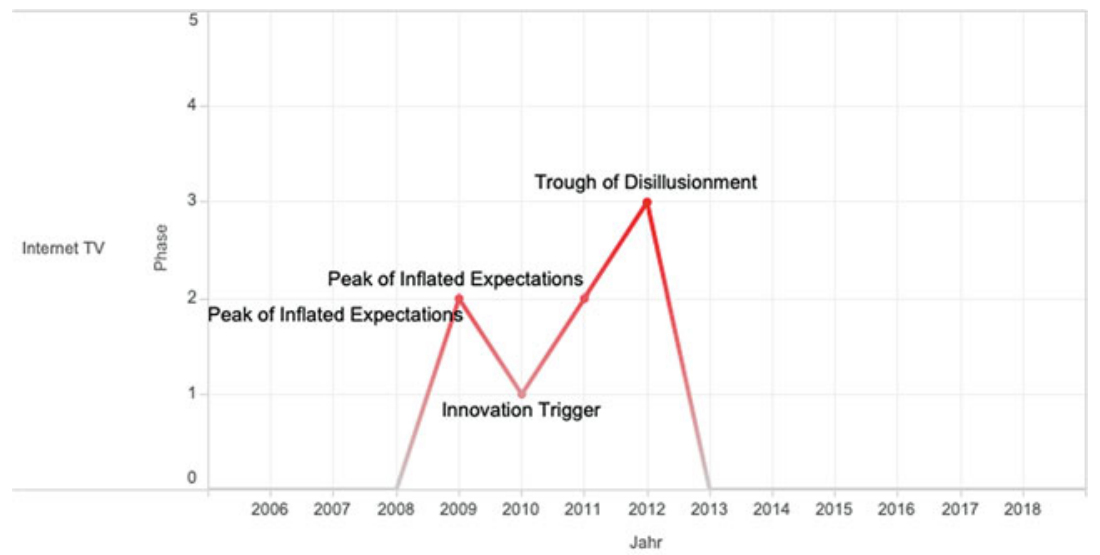

Abb. 5.11 Phasenentwicklung des „Internet TV“ 
TV am Hauptgerät, was gut $7 \%$ entspricht (Statista, 2019). Weltweit sind es 2017 214 Mio. Abonnenten (digital TV research, 2018).

Anhand des dritten Kriteriums, Korrektheit der Prognose, wird geprüft, ob das von Gartner aufgestellte Prognoseschema eingehalten wird. In Abb. 5.12 wird mit der Technologie „Virtual Worlds“ ein Beispiel aufgezeigt. Dabei werden auf der Y-Achse die Jahre bis zum Erreichen des Plateaus und auf der X-Achse die Jahreszahlen angezeigt.

Virtual Worlds beschreibt eine simulierte, technische Umgebung, welche eine Welt schafft, die realitätsferne Zustände ermöglicht (Girvan, 2018, S. 1093). Die Technologie taucht erstmals im Hype Cycle des Jahres 2007 auf. Es wird prognostiziert, dass sie 5-10 Jahre brauchen wird, um das Plateau, Phase 5, zu erreichen. Im Jahr 2008 ist die Technologie wieder enthalten und wird mit 2-5 Jahren prognostiziert. 2010 wird sie wieder mit 5-10 Jahren bewertet, die Prognose wurde folglich korrigiert. Diese Korrektur wird bei diesem Kriterium als falsch bewertet. Die Prognose 5-10 Jahre wird auch für 2010 bis 2012 aufgestellt. 2013 wird die Technologie dann nicht mehr im Hype Cycle aufgeführt. Dieser Verlauf zeigt keine klare Struktur und die Prognose musste korrigiert werden. Korrekt wäre die Prognose verlaufen, wenn Gartner im Jahr 2007 das Erreichen des Plateaus mit 5-10 Jahren, 2008 und 2009 mit 2-5 Jahren und ab 2010 mit kleiner 2 Jahren bewertet hätte. Wäre die Prognose kontinuierlich mit den voranschreitenden Jahren gesunken, wäre die Prognose korrekt gewesen.

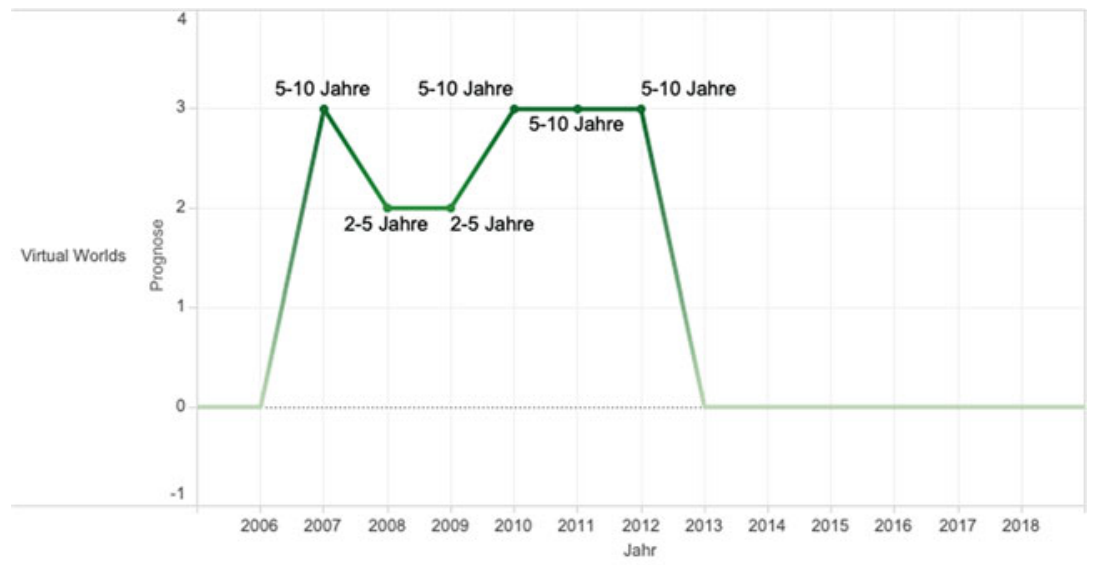

Abb. 5.12 Prognose Virtual Worlds 


\section{Diskussion der Ergebnisse}

Die in Tab. A.2 dargestellten Ergebnisse zeigen, dass 33,6 \% der untersuchten Technologien innerhalb der quantitativ untersuchten Kriterien aufweisen. Daraus lässt sich folgern, dass eine Zeitpunktbetrachtung einzelner Hype Cycle Jahre risikobehaftet sein kann. Es sollte daher bei Betrachtung der Hype Cycles immer ein Zeitraum und kein einzelner Zeitpunkt betrachtet werden, wenn eine Technologie zuverlässiger eingeschätzt werden soll. Zudem empfiehlt es sich, bei der Bewertung einer Technologie nicht nur den Gartner Hype Cycle heranzuziehen, sondern die Einschätzungen mit anderen Quellen zu dieser Technologie zu ergänzen. Nachfolgend werden die untersuchten Kriterien im Einzelnen diskutiert.

Anhand des ersten Kriteriums wird das Vorkommen der Technologien in den Hype Cycles von 2005-2019 analysiert. Dabei ist auffällig, dass 25 der untersuchten Technologien in ihrem Vorkommen eine Inkonsistenz aufweisen. Dieses inkonsistente Vorkommen, das anhand eines Beispiels (siehe Abb. 5.10) aufgezeigt wurde, hat Auswirkungen auf die Aussagekraft der Prognosen des Gartner Hype Cycles. Die Nachvollziehbarkeit der Prognosen und Entwicklungen der Technologien leidet unter diesen Schwankungen. Eine zeitpunktbezogene Betrachtung einer Technologie ist aufgrund des inkonsistenten Vorkommens wenig aussagekräftig. Die Betrachtung einer Technologie sollte deshalb langfristig erfolgen. Das hat den Vorteil, dass einzelne Inkonsistenzen einen geringeren negativen Effekt auf die Aussagekraft der Technologieentwicklung haben. Orientieren sich Unternehmen am Gartner Hype Cycle for Emerging Technologies, so können die Schwankungen diese Unternehmen verunsichern in bestimmte Technologien zu investieren.

Das zweite Kriterium analysiert den Verlauf der Phasen einer Technologie über einen Zeitraum. Dabei sind Technologien aufgetreten, die eine Phasenrückentwicklung beziehungsweise einen doppelten Phasensprung aufweisen. Die Phasenentwicklung gibt dem Betrachter des Hype Cycles die Möglichkeit, die Erwartungen an die Technologie einzuordnen. Zudem kann die Realitätsnähe der Einsatzgebiete einer Technologie anhand der Phaseneinordnung analysiert werden. Beispielsweise sind die zahlreichen Einsatzgebiete während der zweiten Phase oftmals realitätsfern und überzogen. Eine Phasenrückentwicklung ist grundsätzlich fragwürdig, da diese einer Korrektur der Einordnung des Vorjahrs gleichzusetzen ist. Zudem stellt eine Phasenrückentwicklung ein Risiko für Investitionen dar, da die Einordnung der Technologie und die Ermittlung der Einsatzgebiete erschwert werden. Das gleiche Risiko besteht durch das Auslassen einer Phase, beispielsweise bei einem Sprung von Phase 1 in Phase 3. Unternehmen, die sich am Hype Cycle orientieren, werden durch die fragwürdigen Phasenentwicklungen verunsichert und die Aussagekraft des Hype Cycles sinkt. Bei diesem Kriterium ist es sinnvoll eine zeitraumbezogene Betrachtung des Hype Cycles durchzuführen. Ein Vorteil ist, 
dass die konstante Beobachtung der Phasenentwicklung eine Tendenz aufzeigen kann, wie sich die Technologie entwickeln wird. So ist eine Technologie mit Phasenrückentwicklungen (siehe Abb. 5.10), beispielsweise schwer einzuschätzen und eine riskantere Investitionsmöglichkeit als eine Technologie, die einen konstanten Phasenverlauf aufweist.

Das dritte Kriterium befasst sich mit der Korrektheit der Prognose. Wird das Erreichen des Plateaus falsch prognostiziert, kann dies zu verschiedenen Problemen führen. Unternehmen können ihre Forschung auf diese Prognose auslegen. Wird das Erreichen des Plateaus heruntergestuft, beispielsweise von 5-10 Jahre auf $<2$, ist es mit Herausforderungen verbunden, die Technologie in dieser Zeit marktreif zu entwickeln. So kann ein Unternehmen den Marktstart verpassen und teure Investitionen, beispielsweise in Forschung und Entwicklung, könnten vergebens gewesen sein. Wird eine Prognose verlängert, kann dies ebenfalls negative Folgen haben. Wenn ein Unternehmen seit 10 Jahren auf einen Trend hinarbeitet und Gartner nach 10 Jahren die Prognose hochstuft, wurde ein Produkt entwickelt, dessen Markt noch nicht gereift ist. Dadurch entsteht ein Problem für das Unternehmen, da mit der Einführung des Produkts gerechnet wurde, aber die Verzögerung den Marktstart verschiebt. Wird das Produkt trotzdem eingeführt, besteht die Gefahr, dass es sich nicht durchsetzt. Wartet das Unternehmen ab, geht es das Risiko ein, wegen der hohen Investitionsausgaben und einem Ausbleiben von Einnahmen, in finanzielle Probleme zu geraten.

Zur Ermittlung des Gesamtergebnisses wurde das vorher beschriebene Schema mit drei quantitativen Kriterien erstellt. Als Voraussetzung für dieses Schema wurde definiert, dass eine Technologie mindestens zwei Mal in den Gartner Hype Cycles zwischen 2005 und 2019 vorhanden sein muss. Es gibt jedoch Technologien, die nur einmalig im Hype Cycle vorkommen, wie beispielsweise HTML 5 oder das Smartphone. Der Smartphone-Trend ist in Abb. 5.13 dargestellt:

Wie Abb. 5.13 zeigt, wird das Smartphone im Jahr 2006 als Trend in den Hype Cycle aufgenommen und kommt nur dieses eine Mal vor. Allerdings wurde durch Nokia bereits 1996 das erste Smartphone, das Nokia 9000, vorgestellt. Das damals vorgestellte Gerät war portabel und konnte bereits E-Mails und Fax empfangen und versenden, bot Telefonie und die Möglichkeit, im Web zu surfen (Troianovski \& Grundberg, 2012). Allerdings greift Gartner den Trend erst auf, als bereits 64 Mio. Smartphones im Jahr 2006 verkauft wurden. Marken wie Nokia, RIM, Motorola oder Palm hatten bereits funktionstüchtige und etablierte Smartphones auf dem Markt (Canalys.com Ltd., 2007). Diese Smartphones zeichneten sich durch eine Tastatur aus, was das 2006 angekündigte iPhone ändern sollte (Silver, 2018). Es wurde 2007 ausgeliefert und die Verkaufszahlen von Smartphones stiegen insgesamt an (Statista, 2020; IDC Corp., 2020). Jedoch war das iPhone nicht das erste Smartphone 


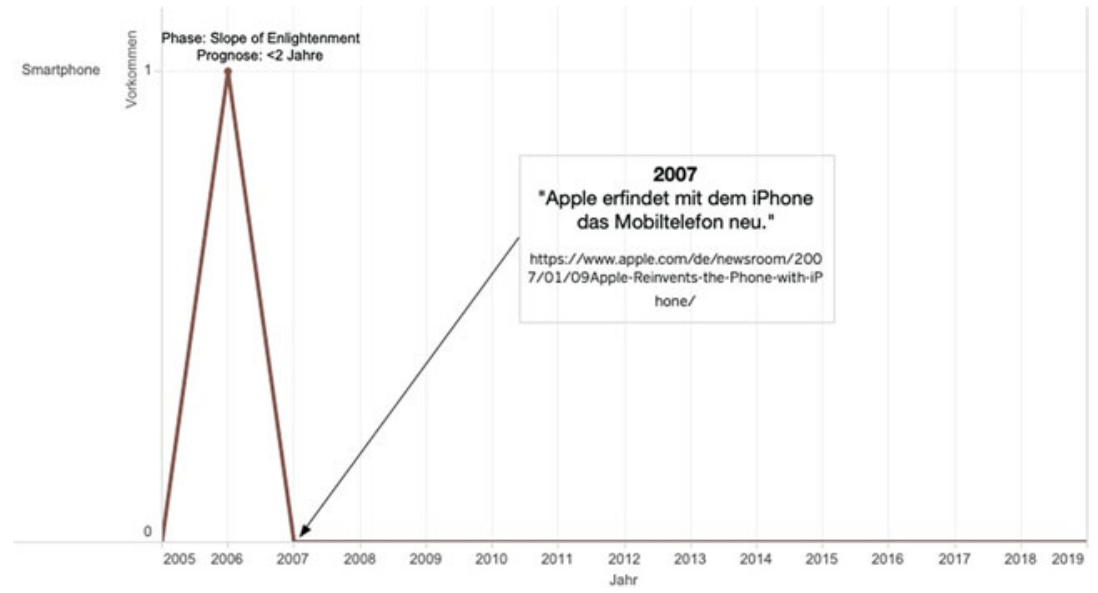

Abb.5.13 Das Smartphone im Gartner Hype Cycle

mit einem farbigen Touchscreen, ein solches hatte Nokia bereits sieben Jahre zuvor vorgestellt (Troianovski \& Grundberg, 2012, S. 1). Gartner wartete folglich entweder gezielt die Ankündigung des iPhones ab, bevor sie die Technologie in den Hype Cycle aufnahmen oder es wurde zu passiv gehandelt und die Technologie wurde nicht als relevant angesehen. Wie in Abb. $5.13 \mathrm{zu}$ erkennen ist, befindet sich der Trend bereits in der Phase Slope of Enlightenment und wird mit <2 Jahren bis zum Erreichen des Plateaus bewertet. Hierbei löste scheinbar erst die Verschmelzung des erfolgreichen Apple iPods mit einem Smartphone den Impuls aus, der Gartner davon überzeugte, die Technologie aufzunehmen. Dabei wurde das Smartphone, wie es von Apple neu geschaffen wurde, so erfolgreich, dass es den Marktführer Nokia, der 2007 einen Marktanteil von $49 \%$ hatte, bis 2013 komplett aus dem Markt verdrängt hat. 2011 lag der Marktanteil von Nokia nur noch bei 15,6\%, der von Apple hingegen bei 18,8 \% (Statista, 2020; IDC Corp., 2020). Ob ein früheres Erscheinen der Technologie im Gartner Hype Cycle Nokia vor dieser Entwicklung bewahrt hätte, kann an dieser Stelle nicht beantwortet werden. Es hätte jedoch entsprechende Impulse geben können.

In diesem Abschnitt erfolgt die Analyse der Gartner Hype Cycles anhand qualitativer Kriterien. Das erste untersuchte Kriterium ist die Einheitlichkeit der Technologie-Bezeichnungen in den Hype Cycles. Insgesamt fällt auf, dass die Bezeichnungen nicht immer konsistent sind. So gibt es einige Technologien, die im Verlauf ihres Vorkommens umbenannt werden oder uneinheitlich benannt sind. 
Konkrete Beispiele hierfür sind dem Anhang zu entnehmen. Ein weiteres Kriterium ist die durchgängig einheitliche Klassifizierung der Technologietrends nach der Prognose. So gibt es nur eine Technologie, die mit der Prognose „obsolete before plateau" klassifiziert wird. Es stellt sich die Frage, wie die Trends einzuschätzen sind, die nicht in diese Prognose-Klasse eingestuft wurden, aber in den Hype Cycles nie das Plateau erreichen. Dieses Verhalten macht es unmöglich, für diese Technologien die Richtigkeit der Prognose zu bewerten, da die Prognose für das Erreichen des Plateaus nicht überprüft werden kann. Auch die Überschrift der Legende ist nicht einheitlich. Während die Legende im Hype Cycle des Jahres 2005 mit der Überschrift „Key: Time to Plateaus“ versehen war, hieß die Überschrift von 2006 bis 2011 Years to mainstream adoption. Ab 2012 lautete die Überschrift „Plateau will be reached“. Ein weiteres qualitatives Kriterium ist die Einheitlichkeit der Achsenbeschriftungen. Von 2005 bis 2008 wird die Y-Achse mit „,visibility“, ab 2009 mit ,expectations“ bezeichnet. Die beschriebenen Inkonsistenzen erschweren es dem Leser, die Entwicklung von Technologietrends über mehrere Jahre hinweg zu verfolgen, vor allem wenn die dazugehörigen kostenpflichtigen Gartner Reports nicht vorliegen.

Die Auswertung der Gartner Hype Cycles beschränkt sich auf die frei zugänglichen Darstellungen in Form von Grafiken. Informationen, die darüber hinaus in den vollständigen und kostenpflichtigen Reports erfasst wurden, wie beispielsweise Informationen zum zwischenzeitlichen Verschwinden einzelner Technologien, wurden nicht berücksichtigt. Da die Annahme getätigt wurde, dass nicht jedes Unternehmen, das sich an den Hype Cycles orientiert, die vollständigen Berichte erwirbt, besitzt das dargestellte Ergebnis trotzdem eine entsprechende Relevanz. Des Weiteren wurden aufgrund des begrenzten Umfangs dieser Auswertung die Kriterien lediglich isoliert voneinander untersucht.

Weitere Analysen, in denen die einzelnen Kriterien miteinander verknüpft und untersucht werden, sind denkbar. Beispielsweise könnte das Phasen-Kriterium und das Prognose-Kriterium miteinander untersucht werden, sodass sich bei fortschreitendem Phasenverlauf auch die Prognose beziehungsweise die Dauer bis zum Erreichen des Produktivitätsplateaus entsprechend verringert. Durch den vorgestellten, teilautomatisierten Ansatz ist es künftig möglich, weitere Jahre des Hype Cycles zu dieser Auswertung hinzuzufügen, was neben der Verknüpfung von Kriterien weiteres Forschungspotenzial bietet.

\section{Fazit}

Die in dieser Arbeit durchgeführte Analyse der Gartner Hype Cycles for Emerging Technologies führt zu dem Ergebnis, dass 66,4 \% der Technologien bezüglich der untersuchten Kriterien Vorkommen, korrekter Phasenverlauf und korrekte Prognose 
als richtig einzustufen sind. Die Analyse anhand der qualitativen Kriterien zeigt, dass die Hype Cycles Inkonsistenzen bezüglich der Einheitlichkeit aufweisen. Dies erschwert die Betrachtung der Technologieentwicklungen.

Auch Dedehayir und Steiner kamen zu dem Ergebnis, dass die Gartner Hype Cycles Unstimmigkeiten aufweisen (Dedehayir \& Steinert, 2016, S. 29). Allerdings ist diese Analyse auf den Zeitraum von 2003 bis 2009 beschränkt. Die in dieser Arbeit durchgeführte Analyse bezieht mit 15 Jahren hingegen einen größeren Zeitraum und auch aktuellere Technologietrends mit ein.

Insgesamt lässt sich festhalten, dass die Gartner Hype Cycles for Emerging Technologies dafür geeignet sind, sich einen Überblick über aktuelle Technologietrends zu verschaffen. Durch die Betrachtung der Hype Cycles über längere Zeiträume hinweg sind Tendenzen bezüglich der weiteren Entwicklung der Trends erkennbar. Um jedoch zuverlässig detaillierte Prognosen für bestimmt Technologien zu stellen, müssen weitere Quellen miteinbezogen werden.

Diese Arbeit liefert eine faktenbasierte Auswertung der Gartner Hype Cycles. Dies erhöht die Objektivität der Ergebnisse, da keine subjektiven Vermutungen angestellt werden. Die teilautomatisierte Vorgehensweise zur Analyse der Gartner Hype Cycles anhand der quantitativen Kriterien verringert die Fehleranfälligkeit gegenüber einer manuellen Auswertung. Dies erhöht die Reliabilität der Analyse.

Die zu den Gartner Hype Cycles dazugehörigen kostenpflichtigen GartnerReports wurden bei der Auswertung nicht miteinbezogen. Eine weitere Einschränkung der in dieser Analyse angewandten Vorgehensweise ist, dass die quantitativen Kriterien einzeln nacheinander geprüft werden, nicht aber in gleichzeitiger Kombination.

Die teilweise automatisierte Auswertung erhöht die Skalierbarkeit, sodass künftig mit geringem Aufwand weitere Hype Cycles analysiert werden können. Auch die Erweiterung der Auswertung um weitere Untersuchungskriterien ist möglich. Außerdem kann in einer weitergehenden Untersuchung der Detailgrad der Analyse erhöht werden, z. B. indem mehrere quantitative Kriterien zugleich miteinander kombiniert werden. Diese Arbeit bietet großes Erweiterungspotenzial, das beispielsweise in einer Roadmap von weiteren Analysen umgesetzt werden könnte. 


\subsection{Big Data - Möglichkeiten und Grenzen der digitalen Phänotypisierung zur Erkennung dementieller Symptome}

Vorwort des Betreuers: Till Hänisch

Was in der Werbung funktioniert - passgenaue Empfehlungen für jeden einzelnen auf der Basis gesammelter Daten - kann auch in der Medizin eingesetzt werden. Zunächst in der Diagnostik, später auf dem Weg von Standardwerten und Leitlinien zur individuellen Therapie, können Technologien wie Big Data und Machine Learning in der Medizin künftig eine wichtige Rolle spielen. Die Funktionsfähigkeit dieser Algorithmen wurde bereits in zahlreichen Beispielen nachgewiesen, etwa in der Radiologie, der Früherkennung von psychischen Störungen oder der Prognose von kardiologischen Notfällen. In Forschungsprojekten wird die Wirksamkeit beim Management chronischer Erkrankungen untersucht. Im folgenden Artikel wird am Beispiel der Demenz dargestellt, wie solche Algorithmen zur Früherkennung dementieller Erkrankungen eingesetzt werden können.

Natürlich spielen hier nicht nur technologische Aspekte, sondern auch Datenschutz, juristische Verifizierbarkeit und vor allem die Akzeptanz der potenziellen Nutzer*innen eine entscheidende Rolle.

Felix Hasselblatt und Alexander Vydrenko

\section{Grundlagen der digitalen Phänotypisierung}

Durch die heute allgegenwärtige und vielfältige Nutzung von sozialen Medien, Foren, Online Communities, digitalen Systemen und mobilen Geräten mit Sensoren, wie beispielsweise Smartphones oder Smartwatches, können für jeden Menschen immer mehr individuelle Daten erhoben und aggregiert werden. Diese Daten ermöglichen die Darstellung eines sehr einzigartigen Musters - des sogenannten Digitalen Phänotyps. Dieser Phänotyp bietet ein unglaubliches Potenzial. Denn durch die Analyse der gesammelten Daten können gesundheitsrelevante Informationen abgeleitet werden. Die so gewonnenen Erkenntnisse helfen in der Folge medizinische Entscheidungen zu treffen bzw. zu unterstützen (Jain et al., 2015).

Hierzu können zum Beispiel mithilfe von Sensoren gemessene Vitalparameter (Puls, Blutdruck, Atemfrequenz, Sauerstoffsättigung, Blutzucker, etc.) der Patient*innen gesammelt und an Gesundheitsdienstleister weitergleitet werden. Daraus werden anschließend nach Analyse und Auswertung Empfehlungen für die weitere medizinische Unterstützung abgeleitet. Die meisten dieser Sensoren sind zu diesem Zweck mit einem internetfähigen Gerät verbunden und können so Daten mit einer zentralen Datenbank austauschen (Abo-Zahhad et al., 2014). 
Sensoren können dabei nicht nur körperliche Parameter der jeweiligen Personen messen (physiologische Sensoren). Auch für die Umgebung steht eine Vielzahl von technischen Messgeräten (Umgebungssensoren) zur Verfügung (Acampora et al., 2013). Mit diesen können unter anderem Umgebungsfaktoren wie Schall, Helligkeit, Raumtemperatur oder Luftfeuchtigkeit gemessen werden. Dabei sind die Sensoren heutzutage so klein, dass ihr Einsatz am und im Körper sowie im Raum kaum noch auffällt. Das Platzieren von Sensoren in Schuhen, Armbanduhren, Matratzen und selbst unter der Haut ist problemlos möglich (Demiris \& Hensel, 2008).

Die digitale Phänotypisierung findet daher mit der Weiterentwicklung der technischen Möglichkeiten im medizinischen Umfeld zunehmend mehr Einzug. Besonders häufig ist sie bereits jetzt schon im Fachgebiet der Psychiatrie anzutreffen. Hier können auf Grundlage der analysierten Daten bestimmte Verhaltensweisen erkannt bzw. gefördert werden. Beispielhaft sei hier die individuelle Belastbarkeit, die Erkennung von Stress oder auch von risikoreichem Alkoholkonsum genannt (Santani et al., 2018; Thomée et al., 2011). Dadurch lassen sich mitunter auch nicht-diagnostizierte Zustände bis hin zur Suizidgefahr proaktiv identifizieren und Rückfälle frühzeitig erkennen bzw. prognostizieren (O'Dea et al., 2017). So bietet sich die Möglichkeit, präventive Maßnahmen rechtzeitig ergreifen zu können (Mundt et al., 2012; Truong et al., 2017). Dies kommt beispielsweise bei Borderline-Persönlichkeitsstörungen oder auch Erkrankungen aus dem schizophrenen Formenkreis zum Tragen (Huckvale et al. 2019). Darüber hinaus ist es mithilfe der digitalen Phänotypisierung möglich, fortschreitende Krankheitsverläufe auszumachen, um die Therapie für einen maximalen Behandlungseffekt zeitnah an die Progredienz bzw. Dynamik der Erkrankung anzupassen (Palmius et al., 2017).

Das digitale Phänotypisieren spielt sich im Bereich Big Data ab, da hierbei sehr große Datenmengen gesammelt und analysiert werden. Der Begriff „Big Data“ beschreibt insgesamt Datensätze, deren Größe die Möglichkeiten typischer Datenbankmanagementsysteme zur Erfassung, Speicherung, Verwaltung und Analyse übersteigen. Je nach Sektor kann die Größe der Daten von einigen Gigabytes bis zu mehreren Petabytes variieren und die Struktur der Daten als strukturiert, semistrukturiert oder unstrukturiert klassifiziert werden (Manyika et al., 2011; Vimarlund \& Wass, 2014).

Dadurch ergibt sich im hier betrachteten Kontext der komplexen Organisationen des Gesundheits- und Sozialwesens die große Herausforderung Daten aus unterschiedlichen Quellen so zu transformieren und auf einer Plattform zu halten, dass eine einzige konsistente Sicht dieser Informationen ermöglicht werden kann. Je nach Herkunft der Daten können diese kategorisiert werden (Demiris \& Hensel, 2008; Vimarlund \& Wass, 2014). Erfasst werden unter anderem Messwerte von 
Sensoren, Messgeräten und anderen Geräten, biometrische Daten wie Fingerabdrücke, medizinische Bildgebungsdaten wie Röntgenbilder, genetische Daten oder die Handschrift und Netzhautscans (Groves et al., 2016).

Die Auswertung der großen Datenmengen erfolgt mithilfe von Methoden künstlicher Intelligenz. Auch wenn diese Technologie sich noch im Anfangsstadium befindet, liefert sie bereits jetzt schon vielversprechende Ergebnisse. Zum Einsatz kommen dabei Methoden wie z. B. Machine Learning, Deep Learning und Natural Language Processing (Graham et al., 2020).

\section{Digitale Phänotypisierung am Beispiel der Demenz}

Das Krankheitsbild der Demenz ist geprägt von erheblichen Einschränkungen in den Aktivitäten des täglichen Lebens und der Lebensqualität. Diese Einschränkungen werden durch den fortschreitenden Abbau kognitiver und intellektueller Fähigkeiten verursacht, die mit Veränderungen des Verhaltens der betroffenen Patient*innen einhergehen (Batsch \& Mittelman, 2015). Die Ursachen von Demenzen können sehr unterschiedlich sein und reichen von Stoffwechselstörungen über Tumorerkrankungen bis hin zu degenerativen Erkrankungen. Da es sich bei der Alzheimer-Demenz um die häufigste Ursache einer demenziellen Symptomatik infolge einer degenerativen Erkrankung handelt, wird diese im Folgenden näher betrachtet.

Nach Angaben der deutschen Alzheimer Gesellschaft waren in Deutschland im Jahr 2018 etwa 1,7 Mio. Menschen von einer Demenz betroffen (Bickel et al., 2018). Prognosen zufolge wird es im Jahr 2050 in Deutschland ca. 3 Mio. Menschen mit einer Demenzerkrankung geben (Bickel et al., 2018; Alzheimer Europe, 2013). Diese angenommene Verdoppelung innerhalb von 32 Jahren zeigt die Dimensionen der zu erwartenden versorgungspolitischen Herausforderungen. Daher betonen medizinische Fachgesellschaften, wie wichtig eine frühzeitige Diagnostik von Demenzerkrankungen ist. Dies nicht zuletzt, da viele therapeutische und präventive Ansätze gerade im Frühstadium der Krankheit eine spätere Pflegebedürftigkeit verzögern können (Deutsche Gesellschaft für Psychiatrie und Psychotherapie, Psychosomatik und Nervenheilkunde, 2017). Verursacht wird diese Pflegebedürftigkeit durch eine Reihe typischer Symptome, welche durch die Demenzerkrankung verursacht werden. Durch den bereits beschriebenen kognitiven Abbau kommt es zunehmend zum Verlust der zeitlich-örtlichen Orientierung, zum Abbau der autobiografischen Identität und im weiteren Verlauf zunehmend auch zu Schwierigkeiten in der Kommunikation sowie zu Veränderungen von Persönlichkeitsmerkmalen (Deutsche Gesellschaft für Psychiatrie und Psychotherapie, Psychosomatik und Nervenheilkunde 2017). Häufig entwickeln Demenzpatient*innen Schlafstörungen, Depressionen und Unruhezustände (Mattle \& Mumenthaler, 2015). Durch die 
Veränderung des Erlebens zeigen viele Betroffene Symptome eines gestörten psychischen Erlebens und Verhaltenssypmtome, wie z. B. Angst, Aggression, lautes Schreien oder Hinlauftendenzen. Die Intensität, Frequenz und Dauer dieses sog. „herausfordernden Verhaltens“ ist sehr unterschiedlich (Savva et al., 2009).

Die Mehrheit der Patient*innen mit einer Demenz weisen motorische Beeinträchtigungen, wie z. B. Gang- und Balancestörungen auf, die in einem frühen Stadium der Erkrankung beginnen und den kognitiven Beeinträchtigungen mindestens um ein Jahrzehnt vorausgehen (Albers et al., 2015). Viele dieser frühzeitigen Symptome sowie die weitaus später auftretenden kognitiven Beeinträchtigungen, lassen sich mithilfe technischer Sensoren erkennen. Hierbei spielen eine ganze Reihe von Werten eine Rolle. Dies können beispielsweise Ganggeschwindigkeit, Schrittlänge und Gangsymmetrie sein (Buracchio et al., 2010). Da diese Werte allein nicht spezifisch genug wären, sind weitere Parameter zur Prädiktion einer möglichen Alzheimer-Demenz heranzuziehen (Case et al., 2015). Diese Parameter können im Alltag mit den verschiedensten Sensoren erfasst werden, um so in Summe eine digitale Phänotypisierung zu ermöglichen. In Bezug auf demenztypische Symptome lassen sich an dieser Stelle als Überblick die folgenden Sensoren eines Smartphones nennen:

- Gyroskop und Beschleunigungssensor

- Gehgeschwindigkeit, Aktivitäten, Tremor (Buracchio et al., 2010; CHAIBUB NETO et al., 2016; Verghese et al., 2008).

- Kamera

- Augenbewegungen, Pupillenreaktion (Albers et al., 2015; Crutcher et al., 2009).

- Touchscreen

- Tipp-Geschwindigkeit, Pausen (Rabinowitz, 2014).

- GPS-Modul

- Fahrverhalten, Bewegung, Aktivitätslevel (Eagle et al., 2009; Saeb et al., 2016).

Zusätzlich zu den genannten Sensoren können Informationen zur allgemeinen Nutzung des Smartphones ausgewertet werden, um mögliche kognitive Veränderungen zu erkennen. Dies kann die Eingabe von PIN-Nummern, Nutzung der Erinnerungsfunktion, die Anzahl der tatsächlich genutzten Apps aber auch die Form und Frequenz der Kommunikation sein (Telefonie, Nachrichten etc.) (Dagum, 2018; Jones et al., 2015).

All diese Informationen könnten langfristig dazu führen, erste Demenzsymptome als digitalen Phänotyp erkennbar bzw. vorhersagbar zu machen. Die 
Schwierigkeit wird darin liegen, Symptome des gesunden Alterungsprozesses von möglicherweise ersten auffälligen zu unterscheiden. Dies wird nur durch langjährige Verlaufsbeobachtungen möglich sein (Opel \& Hahn, 2020).

Den genannten Vorteilen bleiben die Fragestellungen, die sich aus den Bereichen Ethik und Datenschutz ergeben, gegenüber zu stellen.

Offen ist beispielsweise die Frage wo und wie die Daten bei breiterer Anwendung gespeichert und verarbeitet werden können, ohne dass negative Effekte bei unbeabsichtigter Einsichtnahme entstehen. Die Privatsphäre der Nutzer*innen zu schützen wird mit technischen und rechtlichen Regularien nur begrenzt möglich sein. Entwicklungen zu möglicherweise absoluter Sicherheit der Daten (sog. Homomorphe Verschlüsselungsverfahren) befinden sich noch im frühen Entwicklungsstadium (Gentry, 2010). Bis diese Verfahren einen Reifegrad erreicht haben, der sie für medizinische Daten im großen Stil nutzbar macht, wird noch viel Zeit vergehen (Andelfinger \& Hänisch, 2016).

Auch drängen sich viele ethische Fragen auf: Wer wird letztendlich zwischen „gesund“ und „krank“ entscheiden? Ist es eine Ärztin/ein Arzt? Oder wird diese Frage zukünftig von einer Maschine beantwortet? Ab welcher Wahrscheinlichkeit einer Diagnose wird behandelt? Ist eine Behandlung weit vor dem ersten „Leiden“ des Patienten indiziert (Gründer, 2020)? Und nicht zuletzt: Stellen wir unsere Gesundheit so sehr über alles, dass wir dafür solch eine umfassende Überwachung zulassen oder sogar wünschen? Und ist das dann letztendlich noch „gesund“ für den Einzelnen und die Gesellschaft?

\section{Anhang}

Questionnaire

\begin{tabular}{l|l}
\hline No & Question \\
\hline 1 & Could you please introduce yourself? \\
\hline 2 & How do you assess the experience of care institutions in the field of robotics? \\
\hline 3 & $\begin{array}{l}\text { Do you see a general trend towards the use of robots and if this is the case, what is } \\
\text { the acceptance in nursing? }\end{array}$ \\
\hline 4 & $\begin{array}{l}\text { What is the level of acceptance among people in need of care who regularly use } \\
\text { technical products (smartphones, laptops, etc.) in everyday life? }\end{array}$ \\
\hline 5 & $\begin{array}{l}\text { How does the acceptance of patients who have already had contact with technical } \\
\text { solutions from AAL behave? }\end{array}$ \\
\hline
\end{tabular}




\begin{tabular}{l|l}
\hline No & Question \\
\hline 6 & $\begin{array}{l}\text { What is the level of acceptance when carers bring those to be cared for together in } \\
\text { contact with the robots? }\end{array}$ \\
\hline 7 & $\begin{array}{l}\text { How do you judge acceptance if the robots are not used in the intimate area } \\
\text { (approx. } 50 \mathrm{~cm} \text { ) of the person to be cared for? }\end{array}$ \\
\hline 8 & $\begin{array}{l}\text { What effects does the use of social robots have on the social behaviour of the } \\
\text { person in need of care? }\end{array}$ \\
\hline 9 & $\begin{array}{l}\text { Are there differences in the acceptance of robots in a care facility compared to the } \\
\text { home environment? }\end{array}$ \\
\hline 10 & What effects does the appearance (human/technical) of robots have on acceptance? \\
\hline 11 & Would she like to say anything more on the subject? \\
\hline
\end{tabular}

\section{Zusammenführung von Begriffen:}

\begin{tabular}{|c|c|}
\hline Wird geführt als & Zusammengesetzt aus \\
\hline 3D Bioprinting System & $\begin{array}{l}\text { 3D Bioprinting Systems for Organ Transplant }+3 \mathrm{D} \\
\text { Bioprinting System }\end{array}$ \\
\hline Brain-Computer Interface & $\begin{array}{l}\text { Computer-Brain Interface }+ \text { Brain-Computer } \\
\text { Interface }\end{array}$ \\
\hline Networked Collective Intelligence & $\begin{array}{l}\text { Networked Collective Intelligence }+ \text { Collective } \\
\text { Intelligence }\end{array}$ \\
\hline Smart Advisors & Smart Advisors + Cognitive Expert Advisors \\
\hline Mobile Health Monitoring & $\begin{array}{l}\text { Mobile Health Monitoring + Home Health } \\
\text { Monitoring }\end{array}$ \\
\hline SOA & SOA + Service Oriented Architecture (SOA) \\
\hline Location Aware Application & $\begin{array}{l}\text { Location Aware Application + Location "Aware" } \\
\text { Application }\end{array}$ \\
\hline $4 \mathrm{G}$ & $4 \mathrm{G}+4 \mathrm{G}$ Standard \\
\hline Model-Driven Architectures & $\begin{array}{l}\text { Modeldriven Approaches + Model-Driven } \\
\text { Architectures }\end{array}$ \\
\hline Virtual Assistants & Virtual Personal Assistants + Virtual Assistants \\
\hline Virtual Worlds & $\begin{array}{l}\text { Virtual Worlds + Public Virtual Worlds + Virtual } \\
\text { Environments/Virtual Worlds }\end{array}$ \\
\hline 3D Flat-Panel TVs and Displays & $\begin{array}{l}\text { 3-D Flat-Panel Displays + 3D Flat-Panel TVs and } \\
\text { Displays }\end{array}$ \\
\hline Electronic Paper & $\begin{array}{l}\text { Electronic Paper + Electronic Ink/Digital Paper + } \\
\text { Digital Paper/E-Paper }\end{array}$ \\
\hline Big Data & Big Data + "Big Data and Extreme Information" \\
\hline
\end{tabular}




\begin{tabular}{l|l}
\hline Wird geführt als & Zusammengesetzt aus \\
\hline Volumetric Displays & $\begin{array}{l}\text { Volumetric and Holographic Displays + Volumetric } \\
\text { Displays }\end{array}$ \\
\hline Telepresence & Telepresence + Video Telepresence \\
\hline Internet Micropayment Systems & $\begin{array}{l}\text { Internet micro-payments + Internet Micropayment } \\
\text { Systems }\end{array}$ \\
\hline 3D Printing & 3D Printing + 3-D Printing \\
\hline
\end{tabular}

Data Set:

https://1drv.ms/u/s!AhWgRRo3Q6RDhco8diDpAz6-fa7CXw

Kaggle Notebook:

https://1drv.ms/u/s!AhWgRRo3Q6RDhcpbk1Ywk7OJQIAWGw?e=101ziZ

\section{Literatur}

Aaltonen, I., Arvola, A., Heikkilä, P., \& Lammi, H. (2017). Hello Pepper, may I tickle you? Children's and adults' responses to an entertainment robot at a shopping mall. In Proceedings of the Companion of the 2017 ACM/IEEE International Conference on Human-Robot Interaction (S. 53-54).

Abo-Zahhad M, Ahmed SM, Elnahas O. A wireless emergency telemedicine system for patients monitoring and diagnosis. Int J Telemed Appl. 2014; 2014:380787. doi: 10.1155/ 2014/380787. Epub 2014 May 6. PMID: 24883059; PMCID: PMC4026975.

Acampora, G., Cook, D. J., Rashidi, P., \& Vasilakos, A. V. (2013). A survey on ambient intelligence in healthcare. Proceedings of the IEEE, 101(12), 2470-2494.

Agarwal, T. (2018). What is a biosensor - Principle, types of biosensors and their applications. https://www.elprocus.com/what-is-a-biosensor-typesof-biosensors-and-applicati ons/. Zugegriffen: 10. März 2020.

Albers, M. W., Gilmore, G. C., Kaye, J., Murphy, C., Wingfield, A., Bennett, D. A., \& Zhang, L. I. (2015). At the interface of sensory and motor dysfunctions and Alzheimer's disease. Alzheimer's \& Dementia, 11(1), 70-98.

Alzheimer Europe. (2013). Prevalence of dementia in Europe. https://www.alzheimer-eur ope.org/EN/Research/European-Collaboration-on-Dementia/Prevalence-of-dementia/ Prevalence-of-dementia-in-Europe. Zugegriffen: 04. Mai 2020.

Andelfinger, V. P., \& Hänisch, T. (2016). eHealth. Springer Gabler.

Andelfinger, V. P., \& Hänisch, T. (2016). eHealth. Wie Smartphones, Apps und Wearables die Gesundheitsversorgung verändern werden (1. Aufl.). Springer Gabler.

Apple. (2019). ECG app and irregular heart rhythm notification available today on Apple Watch. In: Apple, 27. März 2019. https://www.apple.com/mg/newsroom/2019/03/ecgapp-and-irregular-rhythm-notificationon-apple-watch-available-today-across-europeand-hong-kong/. Zugegriffen: 10. März 2020. 
Archibald, M. M., \& Barnard, A. (2018). Futurism in nursing: Technology, robotics and the fundamentals of care. Journal of Clinical Nursing, 27(11-12), 2473-2480.

Batsch, N. L., \& Mittelman, M. S. (2015). World Alzheimer Report 2012. Overcoming the Stigma of Dementia. Alzheimer's Disease International (ADI), London; 2012. https:// www.alz.co.uk/research/WorldAlzheimerReport2012.pdf. Zugegriffen: 06. Mai 2020.

Becker, H. (2018). Robotik in der Gesundheitsversorgung: Hofnungen, Befürchtungen und Akzeptanz aus Sicht der Nutzerinnen und Nutzer. In O. Bendel (Hrsg.), Pflegeroboter (S. 229-248). Springer Gabler.

Becker, P. (2015). Checkliste Krankheitslehre, (3. Aufl., S. 96) Urban und Fischer.

Bedaf, S. (2015). Overview and categorization of robots supporting independent living of elderly people: What activities do they support and how far have they developed. Assistive Technology, 27(2), 88-100.

Bendel, P. (2019). Pflegeroboter. Gabler Wirtschaftslexikon. https://wirtschaftslexikon.gab ler.de/definition/pflegeroboter-54138/version-368832. Zugegriffen: 07. Jan. 2019.

Bickel, H., Hendlmeier, I., Heßler, J. B., Junge, M. N., Leonhardt-Achilles, S., Weber, J., \& Schäufele, M. (2018). The prevalence of dementia and cognitive impairment in hospitals: Results from the General Hospital Study (GHoSt). Deutsches Ärzteblatt International, 115(44), 733.

Bogner, A. (2014). Interview mit Experten: Eine praxisorientierte Einführung. Wiesbaden: Sprinter VS.

Bortz, J., \& Döring, N. (2006). Forschungsmethoden und Evaluation. Für Human- und Sozialwissenschaftler, mit 87 Tabellen. 4., überarb. Aufl., [Nachdr.]. Heidelberg: SpringerMedizin-Verl. (Springer-Lehrbuch Bachelor, Master).

Bozan, K., \& Berger, A. (2019). Revisiting the technology challenges and proposing enhancements in ambient assisted living for the elderly. In Proceedings of the 52nd Hawaii International Conference on System Sciences.

Braun, A., Holtmannspötter, D., Korte, S., Rijkers-Defrasne, S., \& Zweck, A. (2013). Technologieprognosen. Internationaler Vergleich. 97. VDI Technologiezentrum GmbH. https://www.vditz.de/fileadmin/media/Band_097_Technologieprognosen_Intern ationaler_Vergleich_2013_C1.pdf. Zugegriffen: 17. Juni 2020.

Braun, A., Kirchbuchner, F., \& Wichert, R. (2016). Ambient Assisted Living. In F. Fischer \& A. Krämer (Hrsg.), eHealth in Deutschland - Anforderungen und Potenziale innovativer Versorgungsstrukturen (S. 203-222). Springer Vieweg.

Broadbent, E. (2009). Acceptance of healthcare robots for the older population: Review and future directions. International Journal of Social Robotics, 1(4), 319.

Bundesärztekammer. (2012). VI - 69 Ärztliche und medizinische Versorgung auf dem Lande stärken. https://www.bundesaerztekammer.de/aerztetag/aerztetage-der-vorjahre/ 115-daet-2012-in-nuernberg/beschlussprotokoll/top-vi-taetigkeitsbericht-der-bundesaer ztekammer/gesundheitspolitik/vi-69-aerztliche-und-medizinische-versorgung-auf-demlande-staerken/. Zugegriffen: 05. März 2020.

Bundesärztekammer. (2019). Kassenärztliche Bundesvereinigung (KBV), Arbeitsgemeinschaft der Wissenschaftlichen Medizinischen Fachgesellschaften (AWMF). (2019) Nationale Versorgungsleitlinie Chronische Herzinsuffizienz - Langfassung, (3. Aufl. S. 128). https://doi.org/10.6101/AZQ/000465.

Buracchio, T., Dodge, H. H., Howieson, D., Wasserman, D., \& Kaye, J. (2010). The trajectory of gait speed preceding mild cognitive impairment. Archives of Neurology, 67(8), 980-986. 
BVMed e.V. (2018). Portal für Medizintechnik. Abrechnung Telekardiologie. https://www. bvmed.de/de/technologien/herz-und-herzgefaesse/abrechnung-telekardiologie?pk_cam paign=src_RSS. Zugegriffen: 05. März 2020.

Canalys.com Ltd. (2007). 64 million smart phones shipped worldwide in 2006. https://www. canalys.com/static/press_release/2007/r2007024.pdf. Zugegriffen: 17. Juni 2020.

Case, M. A., Burwick, H. A., Volpp, K. G., \& Patel, M. S. (2015). Accuracy of smartphone applications and wearable devices for tracking physical activity data. JAMA, 313(6), 625626.

CHAIBUB NETO, E. L. I. A. S., Bot, B. M., Perumal, T., Omberg, L., Guinney, J., Kellen, M., ... \& Trister, A. D. (2016). Personalized hypothesis tests for detecting medication response in Parkinson disease patients using iPhone Sensor data. In Biocomputing 2016: Proceedings of the Pacific Symposium (S. 273-284).

Corbin, J. \& Strauss, A. (1990). Grounded theory research: Procedures, canons, and evaluative criteria. Qualitative Sociology, 13 (t), 1990.

Crutcher, M. D., Calhoun-Haney, R., Manzanares, C. M., Lah, J. J., Levey, A. I., \& Zola, S. M. (2009). Eye tracking during a visual paired comparison task as a predictor of early dementia. American Journal of Alzheimer's Disease \& Other Dementias, 24(3), 258-266.

Dagum, P. (2018). Digital biomarkers of cognitive function. NPJ Digital Medicine, 1(1), 1-3.

Dawson, M. (2017). Exploring secure computing for the internet of things, internet of everything, web of things, and hyperconnectivity. In M. Dawson, M. Omar, \& M. Eltayeb, Security solutions for hyperconnectivity and the internet of things (S. 1-12). IGI Global. https://doi.org/10.4018/978-1-5225-9866-4.ch053.

Dedehayir, O., \& Steinert, M. (2016). The hype cycle model: A review and future directions. Technological Forecasting and Social Change, 108(C), S. 28-41. https://doi.org/10.1016/ j.techfore.2016.04.005.

Demiris, G., \& Hensel, B. K. (2008). Technologies for an aging society: A systematic review of "smart home" applications. Yearbook of Medical Informatics, 17(01), 33-40.

Deutsche Gesellschaft für Psychiatrie und Psychotherapie, Psychosomatik und Nervenheilkunde. (2017). Deutsche Gesellschaft für Suchtforschung und Suchttherapie (DG-Sucht), et al. S3-Leitlinie" Screening, Diagnostik und Behandlung des schädlichen und abhängigen Tabakkonsums". AWMF-Register Nr. 076-006. (Stand: 09.02. 2015). 2014 [cited: 2015 Jun 17]. S3-Leitlinie Methamphetaminbezogene Störungen.

digital TV research. (2018). Global Pay TV Forecasts. Lymington, Großbritannien. https:// www.digitaltvresearch.com/ugc/press/236.pdf. Zugegriffen: 17. Juni 2020.

Dijkers, M., deBear, P., Erlandson, R., Kristy, K., Geer, D., \& Nichols, A. (1991). Patient and staff acceptance of robotic technology in occupational therapy: A pilot study. Journal of Rehabilitation Research and Development, 28(2), 33-44.

Eagle, N., Pentland, A. S., \& Lazer, D. (2009). Inferring friendship network structure by using mobile phone data. Proceedings of the National Academy of Sciences, 106(36), $15274-15278$.

Eggert, S., Sulmann, D., \& Teubner, C. (April 2018). Einstellung der Bevölkerung zu digitaler Unterstützung in der Pflege. Von Stiftung ZQP. https://www.zqp.de/wp-content/uploads/ ZQP_Analyse_PflegeDigitalisierung.pdf.

Endruweit, G., Trommsdorff, G., \& Burzan, N. (2014). Wörterbuch der Soziologie. UVK Verlagsgesellschaft mbH. 
Farra, R., Sheppard, N. F., McCabe, L., Neer, R. M., Anderson, J. M., \& Santini, J. T. (2012). First-in-human testing of a wirelessly controlled drug delivery microchip. In: Science Translational Medicine 4(122), 122ra21. https://doi.org/10.1126/scitranslmed.3003276.

Fioranelli, E. 2019. Pflegeroboter: Eine Kosten-Nutzen-Analyse. Von KMA Online - Robotik, Pflege und Gesundheitsökonomie. https://www.kma-online.de/aktuelles/it-digital-health/ detail/pflegeroboter-eine-kosten-nutzen-analyse-a-39557/3. Zugegriffen: 17. Januar 2019.

Flake, R., Kochskämper, S., Risius, P., \& Seyda, S. (2018). Fachkräftemangel in der Altenpflege. iwd - Land unter in der Pflege (S. 2-3). Institut der deutschen Wirtschaft, Köln Medien.

Forlizzi, J. (2005). Robotic products to assist the aging population. Interactions No., 12, $16-18$.

Forsa. (2016). Service-Robotik: MenschTechnik-Interaktion im Alltag. Politik- und Sozialforschung GmbH Berlin.

Frankfurter Allgemeine Zeitung. (2018). Mikrochips im Menschen. In: Frankfurter Allgemeine Zeitung, 23.08.2018. https://www.faz.net/aktuell/wirtschaft/mikrochips-im-men schen-experten-warnen-vorgefahren-15749982.html. Zugegriffen: 10. März 2020.

Freund, T., Gerste, B., \& Jeschke, E. (2016). Qualität der poststationären Arzneimittelversorgung von Patienten mit Herzinsuffizienz. In Klauber J, Geraedts M, Friedrich J, \& Wasem J. (Hrsg.), Krankenhaus-Report (S. 229-246). Schattauer.

Fritsch, M., Henning, T., Slavtchev, V., \& Steigenberger, N. (2008). Hochschulen als regionaler Innovationsmotor? Innovationstransfer aus Hochschulen und seine Bedeutung für die regionale Entwicklung. Hans-Böckler-Stiftung, Düsseldorf. https://EconPapers.repec. org/RePEc:zbw:hbsarb:158. Zugegriffen: 17. Juni 2020.

Gartner Inc. (2005). Hype cycle for emerging technologies, 2005. https://www.gartner.com/ en/documents/484310/hype-cycle-for-emerging-technologies-2005. Zugegriffen: 17. Juni 2020.

Gartner Inc. (2006). Hype cycle for emerging technologies, 2006. https://www.gartner.com/ en/documents/494180/hype-cycle-for-emerging-technologies-2006. Zugegriffen: 17. Juni 2020.

Gartner Inc. (2007). Hype cycle for emerging technologies, 2007. https://www.gartner.com/ en/documents/509710/hype-cycle-for-emerging-technologies-2007. Zugegriffen: 17. Juni 2020.

Gartner Inc. (2008). Hype cycle for emerging technologies, 2008. https://www.gartner.com/ en/documents/717415/hype-cycle-for-emerging-technologies-2008. Zugegriffen: 17. Juni 2020.

Gartner Inc. (2009). Hype cycle for emerging technologies, 2009. https://www.gar tner.com/en/documents/1085912/hype-cycle-for-emerging-technologies-2009. Zugegriffen: 17. Juni 2020.

Gartner Inc. (2010). Hype cycle for emerging technologies, 2010. https://www.gar tner.com/en/documents/1414917/hype-cycle-for-emerging-technologies-2010. Zugegriffen: 17. Juni 2020.

Gartner Inc. (2011). Hype cycle for emerging technologies, 2011. https://www.gar tner.com/en/documents/1754719/hype-cycle-for-emerging-technologies-2011. Zugegriffen: 17. Juni 2020. 
Gartner Inc. (2012). Hype cycle for emerging technologies, 2012. https://www.gar tner.com/en/documents/2100915/hype-cycle-for-emerging-technologies-2012. Zugegriffen: 17. Juni 2020.

Gartner Inc. (2013). Hype cycle for emerging technologies, 2013. https://www.gar tner.com/en/documents/2571624/hype-cycle-for-emerging-technologies-2013. Zugegriffen: 17. Juni 2020.

Gartner Inc. (2014). Digital business technologies dominate gartner 2014 emerging technologies hype cycle. https://www.forbes.com/sites/gartnergroup/2014/09/17/digital-businesstechnologies-dominate-gartner-2014-emerging-technologies-hype-cycle/. Zugegriffen: 17. Juni 2020.

Gartner Inc. (2015). What's new in gartner's hype cycle for emerging technologies. https://www.gartner.com/smarterwithgartner/whats-new-in-gartners-hype-cyclefor-emerging-technologies-2015/. Zugegriffen: 17. Juni 2020.

Gartner Inc. (2016). 3 Trends appear in the gartner hype cycle for emerging technologies. https://www.gartner.com/smarterwithgartner/3-trends-appear-in-the-gartner-hypecycle-for-emerging-technologies-2016/. Zugegriffen: 17. Juni 2020.

Gartner Inc. (2017). Top trends in the gartner hype cycle for emerging technologies. https://www.gartner.com/smarterwithgartner/top-trends-in-the-gartner-hype-cyclefor-emerging-technologies-2017/. Zugegriffen: 17. Juni 2020.

Gartner Inc. (2018). 5 Trends emerge in the gartner hype cycle for emerging technologies. https://www.gartner.com/smarterwithgartner/5-trends-emerge-in-gartner-hypecycle-for-emerging-technologies-2018/. Zugegriffen: 17. Juni 2020.

Gartner Inc. (2019). 5 Trends appear on the gartner hype cycle for emerging technologies. https://www.gartner.com/smarterwithgartner/5-trends-appear-on-the-gartner-hypecycle-for-emerging-technologies-2019/. Zugegriffen: 17. Juni 2020.

Gartner Inc. (2020). Research \& advisory overview. https://www.gartner.com/en/research/ methodologies. Zugegriffen: 17. Juni 2020.

Gasser, T., Lerche, H., \& Ziemann, U. (2019). Personalisierte Diagnostik und Therapie in der Neurologie: Chance und Herausforderung. Der Nervenarzt, 90(8), 765-766. https://doi. org/10.1007/s00115-019-0757-8.

Gentry, C. (2010). Computing arbitrary functions of encrypted data. Communications of the ACM, 53(3), 97-105.

Giersch, S., Zenkner, H., \& Härter, H. (2019). Wearables: Mit Optoelektronik und Pulsoximetrie die Herzfrequenz bestimmen. https://www.elektronikpraxis.vogel.de/wearablesmit-optoelektronik-und-pulsoximetriedie-herzfrequenz-bestimmen-a-869243/. Zugegriffen:10. März 2020.

Girvan, C. (2018). What is a virtual world? Definition and classification. Educational Technology Research and Development, 66, 1087-1100. https://doi.org/10.1007/s11423-0189577-y.

Graham, S. A., Lee, E. E., Jeste, D. V., Van Patten, R., Twamley, E. W., Nebeker, C., ... \& Depp, C. A. (2020). Artificial intelligence approaches to predicting and detecting cognitive decline in older adults: A conceptual review. Psychiatry Research, 284, 112732.

Groves, P., Kayyali, B., Knott, D., \& Kuiken, S. V. (2016). The 'big data' revolution in healthcare: Accelerating value and innovation. https://www.mckinsey.com/insights/health_ systems/ /media/7764A72F70184C8EA88D805092D72D58.ashx. Zugegriffen: 30. Mai 2020. 
Gründer, G. (2020). Digitale Phänotypisierung. Kontra. Der Nervenarzt, 91(9), 860-862.

Hall, E. T. (1966). The hidden dimension. Doubleday.

Haun, M. (2006). Handbuch der Robotik. Springer.

Heidbuchel, H., Hindricks, G., Broadhurst, P., Van Erven, L., Fernandez-Lozano, I., RiveroAyerza, M., \& Annemans, L. (2015). EuroEco (European Health Economic Trial on Home Monitoring in ICD Patients): A provider perspective in five European countries on costs and net financial impact of follow-up with or without remote monitoring. European Heart Journal, 36(3), 158-169.

Huckvale, K., Venkatesh, S., \& Christensen, H. (2019). Toward clinical digital phenotyping: A timely opportunity to consider purpose, quality, and safety. NPJ Digital Medicine, 2(1), $1-11$.

Humbert, A. (2019). Wie Du mit Herzratenvariabilität (HRV) einfach und schnell Dein StressLevel messen kannst. In: Mein Weg aus der Angst, 26.05.2019. https://www.meinwegau sderangst.de/herzfrequenzvariabilitaet-hrv-stressmessen/. Zugegriffen: 10. März 2020.

Hüttemann, D. (2018). Typ-1-Diabetes: Vollautomatische Pumpe verbessert Blutzucker. Avoxa - Mediengruppe Deutscher Apotheker GmbH. https://www.pharmazeutische-zei tung.de/vollautomatische-pumpe-verbessert-blutzucker/. Zugegriffen: 10. März 2020.

IDC Corp. (2020). Apple Takes Top Spot in Q4 2019 Worldwide Smartphone Market While Huawei Rises to Number 2 Globally for 2019. Framingham. https://www.idc.com/getdoc. jsp?containerId=prUS45964220. Zugegriffen: 17. Juni 2020.

IDG Business Media GmbH .(2005). Gartner hype cycle for emerging technologies 2005. https://images.cio.de/bdb/2572216/1066x600.webp. Zugegriffen: 17. Juni 2020.

IDG Business Media GmbH. (2006). Gartner hype cycle for emerging technologies 2006. https://images.cio.de/bdb/2572217/1066x600.webp. Zugegriffen: 17. Juni 2020.

IDG Business Media GmbH. (2007). Gartner hype cycle for emerging technologies 2007. https://images.cio.de/bdb/2572218/1066x600.webp. Zugegriffen: 17. Juni 2020.

IDG Business Media GmbH. (2008). Gartner hype cycle for emerging technologies 2008. https://images.cio.de/bdb/2572219/1066x600.webp. Zugegriffen: 17. Juni 2020.

IDG Business Media GmbH. (2009). Gartner hype cycle for emerging technologies 2009. https://images.cio.de/bdb/2572220/1066x600.webp. Zugegriffen: 17. Juni 2020.

IDG Business Media GmbH. (2010). Gartner hype cycle for emerging technologies 2010. https://images.cio.de/bdb/2572221/1066x600.webp. Zugegriffen: 17. Juni 2020.

IDG Business Media GmbH. (2011). Gartner hype cycle for emerging technologies 2011. https://images.cio.de/bdb/2572222/1066x600.webp. Zugegriffen: 17. Juni 2020.

IDG Business Media GmbH. (2012). Gartner hype cycle for emerging technologies 2012. https://images.cio.de/bdb/2572223/1066x600.webp. Zugegriffen: 17. Juni 2020.

IDG Business Media GmbH. (2013). Gartner Hype Cycle of Emerging Technologies 2013. https://images.cio.de/bdb/2572224/1066x600.webp. Zugegriffen: 17. Juni 2020.

Ip, R., Michael, K., \& Michael, M. G. (2008). Amal Graafstra- The Do-It-Yourselfer RFID Implantee: The culture, values and ethics of hobbyist implantees. https://www.aca demia.edu/26134340/Amal_Graafstra-_The_Do-It-Yourselfer_RFID_Implantee_The_ culture_values_and_ethics_of_hobbyist_implantees. Zugegriffen: 10. März 2020.

IQTIG-Institut. (2017). Jahresbericht 2017 des Deutschen Herzschrittmacher- und Defibrillator-Registers. https://pacemaker-register.de/wpcontent/uploads/Jahresbericht2017-des-Deutschen-Herzschrittmacher-und-Defibrillatorregister-Teil-1-Herzschrittm acher.pdf. Zugegriffen: 10. März 2020. 
Isakadze, N., \& Martin, S. S. (2019). How useful is the smartwatch ECG? In: Trends in Cardiovascular Medicine. https://doi.org/10.1016/j.tcm.2019.10.010.

Jain, S. H., Powers, B. W., Hawkins, J. B., \& Brownstein, J. S. (2015). The digital phenotype. Nature Biotechnology, 33(5), 462-463.

Jakobs, J. (2019). Hackerangriffe auf Herzschrittmacher? Ärzte Zeitung (S. 11). https:// www.wiso-net.de/document/AEZT_cd7a628313dbaeaa9c0f3d661fd46b97ed7f6dd6. Zugegriffen: 05. März 2020.

Jones, S. L., Ferreira, D., Hosio, S., Goncalves, J., \& Kostakos, V. (2015, September). Revisitation analysis of smartphone app use. In Proceedings of the 2015 ACM International Joint Conference on Pervasive and Ubiquitous Computing (S. 1197-1208).

Kehl, C. (2018). TAB-Arbietsbericht Nr. 177 - Robotik und assistive Neurotechnologien in der Pflege - gesellschaftliche Herausforderungen. Büro für Technikfolgen-Abschätzung beim deutschen Bundestag.

Kirschenbauer, F. (2018). Connected Healthcare: Medizinische Wearables - Electronica Blog. https://blog.electronica.de/2018/11/08/connected-healthcaremedizinische-wea rables/. Zugegriffen:10. März 2020.

Koay, K. L., Syrdal, D. S., Walters, M. L., \& Dautenhahn, K. (2007). Living with robots: Investigating the habituation effect in participants' preferences during a longitudinal human-robot interaction study. Proceedings of the 16th IEEE International Workshop on Robot and Human Interactive Communication (RO-MAN 2007), (S. 564-569). Jeju, Korea.

Kochskämpfer, S. (2018). Die Entwicklung der Pflegefallzahlen in den Bundefsländern-EIne Simulation bis 2035. Institut der deutschen Wirtschaft.

Koehler, F., Koehler, K., Deckwart, O., Prescher, S., Wegscheider, K., Kirwan, B. A., \& Stangl, K. (2018). Efficacy of telemedical interventional management in patients with heart failure (TIM-HF2): A randomised, controlled, parallel-group, unmasked trial. The Lancet, 392(10152), 1047-1057.

Köhler, F., \& Lücke, S. (2007). Partnership for the Heart: Klinische Erprobung eines telemedizinischen Betreuungssystems für Patienten mit chronischer Herzinsuffizienz. Kardiotechnik, 4, 110-113.

Korn, O. (2019). Soziale Roboter - Einführung und Potenziale für Pflege und Gesundheit. Springer Fachmedien Wiesbaden GmbH.

Kotb, A., Cameron, C., Hsieh, S., \& Wells, G. (2015). Comparative effectiveness of different forms of telemedicine for individuals with heart failure (HF): A systematic review and network meta-analysis. PLoS One, 10 (2), 1-15. https://doi.org/10.1371/journal.pone.011 8681.

Kötter, J. (2017). ePatientenakte: Wer bekommt die Datenhoheit? Hg. v. Der Hausarzt. Digital. https://www.hausarzt.digital/politik/epatientenaktewer-bekommt-die-datenh oheit-22376.html. Zugegriffen: 10. März 2020.

Kreutzer, R. T. (2015). Digitale Revolution - Auswirkungen auf das Marketing. Springer Gabler. https://doi.org/10.1007/978-3-658-09394-5

Krüger-Brand, E. H. (2016). Datenschutz im Gesundheitswesen: Viele Neuregelungen stehen bevor. Deutsches Ärzteblatt, 113(6), 218.

Kumar, R. S. (2018). Robotic nursing in health care delivery. International Journal of Nursing Education, 10(3), 148-151. 
Kurz, C. (2016). Cyborgs! Interview über Implantate, Ende-zu-Ende-Verschlüsselung und Menschenwürde. https://netzpolitik.org/2016/cyborgs-interview-ueber-implantate-endezu-endeverschluesselung-und-menschenwuerde/. Zugegriffen: 10. März 2020.

Kurz, K. (2016). Immens hoher Innovationsdruck. Industrie anzeiger. https://industrieanz eiger.industrie.de/management/immens-hoher-innovationsdruck/. Zugegriffen: 17. Juni 2020.

Leistner, A. (2018). Körper-Upgrade: Bis zu 3.500 Deutsche tragen eingepflanzte Mikrochips. In: Euronews, 06.06.2018. https://de.euronews.com/2018/06/06/eingepflanzte-mik rochips-deutschland. Zugegriffen: 10. März 2020.

Linden, A., \& Fenn, J. (2003). Understanding Gartner's Hype Cycles. Strategic Analysis Report. http://www.ask-force.org/web/Discourse/Linden-HypeCycle-2003.pdf. Zugegriffen: 17. Juni 2020.

Manyika J., Chui M., Brown B., Bughin, J., Dobbs, R., Roxburgh, C., \& Byers, A. H. (2011). Big data: The next frontier for innovation, competition, and productivity. McKinsey Global Institute. https://www.mckinsey.com/ /media/McKinsey/Business\%20F unctions/McKinsey\%20Digital/Our\%20Insights/Big\%20data\%20The\%20next\%20fron tier\%20for\%20innovation/MGI_big_data_full_report.ashx. Zugegriffen: 30. Mai 2020.

Mattle, H., \& Mumenthaler, M. (2015). Kurzlehrbuch Neurologie. Georg Thieme.

Mayer, P. J., Weghofer, A., \& Mut, M. (2018). Needs assessment for active and assisted livingconcepts among the elderly. Gebäude der Zukunft, 269-274. http://hdl.handle.net/20.500. 11790/1120. Zugegriffen: 15. Juli 2021.

McKinsey \& Company. (2018). Digitalisierung in deutschen Krankenhäusern. https:// www.mckinsey.de/ /media/mckinsey/locations/europe $\% 20$ and $\% 20$ middle $\% 20$ east/deu tschland/publikationen/digitalisierung $\% 20$ chance $\% 20 \mathrm{mit} \% 20 \mathrm{milliardenpotenzial} / \mathrm{upd}$ ate_digitalisierung\%20im\%20krankenhaus_mckinsey_update\%20september\%202018. ashx. Zugegriffen: 05. März 2020.

MEDCERT. (2020). Der Weg zum CE-Zeichen. http://www.medcert.de/der-weg-zum-ce-zei chen/. Zugegriffen: 10. März 2020.

Michael, K., \& Michael, M. G. (2005). Microchipping people: The rise of the electrophorus. https://ro.uow.edu.au/cgi/viewcontent.cgi?article=1373\&context=infopapers. Zugegriffen: 10. März 2020.

Moutafis, J. (2019). Chip-Implantate: Ein Plus an Sicherheit oder purer Leichtsinn? In: Business User, 21.01.2019. https://business-user.de/workplace/chip-implantate-ein-plusan-sicherheit-oder-purer-leichtsinn/. Zugegriffen: 10. März 2020.

Mundt, J. C., Vogel, A. P., Feltner, D. E., \& Lenderking, W. R. (2012). Vocal acoustic biomarkers of depression severity and treatment response. Biological Psychiatry, 72(7), 580-587.

Nagel, T. S. (2019). Medizin-Wearables: Was taugen die neuen Gesundheitsgeräte? In: WELT, 11.03.2019. https://www.welt.de/wirtschaft/webwelt/article190092755/Medizin-Wearab les-Wastaugen-die-neuen-Gesundheitsgeraete.html. Zugegriffen: 10. März 2020.

O’dea, B., Larsen, M. E., Batterham, P. J., Calear, A. L., \& Christensen, H. (2017). A linguistic analysis of suicide-related Twitter posts. Crisis: The Journal of Crisis Intervention and Suicide Prevention, 38(5), 319.

Opel, N., \& Hahn, T. (2020). Digitale Phänotypisierung. Pro. Der Nervenarzt, 91(9), 857-859. 
Palmius, N., Tsanas, A., Saunders, K. E. A., Bilderbeck, A. C., Geddes, J. R., Goodwin, G. M., \& De Vos, M. (2016). Detecting bipolar depression from geographic location data. IEEE Transactions on Biomedical Engineering, 64(8), 1761-1771.

Pandey, A. K., \& Gelin, R. (2018). A mass-produced sociable humanoid robot: Pepper: The first machine of its kind. IEEE Robotics \& Automation Magazine, 25(3), 40-48.

Pfannstiel, M. A., Krammer, S., \& Swoboda, W. (2018). Digitale Transformation von Dienstleistungen im Gesundheitswesen IV - Impulse für die Pflegeorganisation. Springer Gabler.

PwC. (2019). Datensicherheit in Kliniken und Arztpraxen. https://www.pwc.de/de/gesundhei tswesen-und-pharma/pwc-datensicherheit-in-klinikenund-arztpraxen.pdf. Zugegriffen: 10. März 2020.

Rabinowitz, I., \& Lavner, Y. (2014). Association between finger tapping, attention, memory, and cognitive diagnosis in elderly patients. Perceptual and Motor Skills, 119(1), 259-278.

Riedl, N. (2019). Unter Dach und Haut. https://www.rubikon.news/artikel/unter-dach-undhaut. Zugegriffen: 10. März 2020.

Rohleder, B. (2016). Digitalisierung der Medizin und im Gesundheitswesen. Digital Health. https://www.bitkom.org/sites/default/files/pdf/Presse/Anhaenge-an-PIs/2016/BitkomPre ssekonferenz-Digital-Health-15-09-2016-Praesentation-final.pdf. Zugegriffen: 10. März 2020.

Rotolo, D., Hicks, D., \& Martin, B. R. (2015). What is an emerging technology? Research Policy, 44(10), 1827-1843. https://doi.org/10.1016/j.respol.2015.06

Rötzer, F. (2007). Verursachen implantierte RFID-Chips Krebs? In: Heise online, 11.09.2007. https://www.heise.de/tp/features/Verursachenimplantierte-RFID-Chips-Krebs-3415250. html. Zugegriffen: 10. März 2020.

Rövekamp, M. (2019). Mikrochips unter der Haut: Wie die Technik zum Teil des Körpers wird. https://www.tagesspiegel.de/wirtschaft/mikrochipsunter-der-haut-wie-die-technikzum-teil-des-koerpers-wird/25310962.html. Zugegriffen: 10. März 2020.

Saeb, S., Lattie, E. G., Schueller, S. M., Kording, K. P., \& Mohr, D. C. (2016). The relationship between mobile phone location sensor data and depressive symptom severity. PeerJ, 4 , e2537.

Salmen, Y. (2015). Mikrochips versorgen den Körper wohldosiert mit Medizin. https://www. trendsderzukunft.de/mikrochips-versorgen-den-koerperwohldosiert-mit-medizin/. Zugegriffen: 10. März 2020.

Santani, D., Labhart, F., Landolt, S., Kuntsche, E., \& Gatica-Perez, D. (2018). DrinkSense: Characterizing youth drinking behavior using smartphones. IEEE Transactions on Mobile Computing, 17(10), 2279-2292.

Savva, G. M., Zaccai, J., Matthews, F. E., Davidson, J. E., McKeith, I., \& Brayne, C. (2009). Prevalence, correlates and course of behavioural and psychological symptoms of dementia in the population. The British Journal of Psychiatry, 194(3), 212-219.

Schäfer, M., \& Keppler, D. (2013). Modelle der technikorientierten Akzeptanzforschung - Überblick und Reflexion am Beispiel eines Forschungsprojekts zur Implementierung innovativer technischer Energieeffizienz-Maßnahmen. TU Berlin.

Schimpl, B. (2017). Die Herzratenvariabilität (HRV) - Alles, was du wissen musst! https://www.vital-monitor.com/shop/die-hrv-herzratenvariabilitaetalles-was-duwissen-musst/. Zugegriffen: 10. März 2020. 
Schleiter, A. (2014). Innovationsdruck auf Führungskräfte in der Wirtschaft wächst. Unkonventionelle Lösungen werden oft innerbetrieblich ausgebremst. change. (B. Stiftung, Hrsg.) Gütersloh. https://www.bertelsmann-stiftung.de/de/themen/aktuelle-mel dungen/2014/maerz/innovationsdruck-auf-fuehrungskraefte-in-der-wirtschaft-waechst/. Zugegriffen: 17. Juni 2020.

Schwartz, O. (2019). The rise of microchipping: Are we ready for technology to get under the skin? In: The Guardian, https://www.theguardian.com/technology/2019/nov/08/ the-rise-of-microchipping-are-weready-for-technology-to-get-under-the-skin. Zugegriffen: 10. März 2020.

Siegel, E. G., \& Siegel, E G. (2019). Versorgungsstrukturen, Berufsbilder und professionelle Diabetesorganisationen in Deutschland. In: Deutscher Gesundheitsbericht Diabetes, S. 240. https://www.deutsche-diabetesgesellschaft.de/fileadmin/Redakteur/Stellu ngnahmen/Gesundheitspolitik/20181114gesundheitsbericht_2019.pdf . Zugegriffen: 10. März 2020.

Silver, S. (2018). The story of the original iPhone, that nobody thought was possible. AppleInsider. https://appleinsider.com/articles/18/06/29/the-story-of-the-original-iph one-that-nobody-thought-was-possible. Zugegriffen: 17. Juni 2020.

Smith, C. (2008). HUMAN MICROCHIP IMPLANTATION. Journal of Technology Management \& Innovation, 3,(3). https://doi.org/10.4067/S0718-27242008000100015.

SoftBank Robotics Group Corp. (2020). The power of robotics to benefit humanity. https:// softbankrobotics.com/. Zugegriffen: 22. Juli 2020.

statista. (2019). Statistiken zum IPTV. https://de.statista.com/themen/724/iptv/. Zugegriffen: 17. Juni 2020.

statista. (2020). Global smartphone shipments from 2007 to 2019, by vendor. https://www.sta tista.com/statistics/271539/worldwide-shipments-of-leading-smartphone-vendors-since2007/. Zugegriffen: 17. Juni 2020.

Statistisches Bundesamt. (2017). Krankheitskosten - Herz-Kreislauf-Erkrankungen verursachen die höchsten Kosten. https://www.destatis.de/DE/Themen/Gesellschaft-Umwelt/ Gesundheit/Krankheitskosten/_inhalt.html. Zugegriffen: 05. März 2020.

Statistisches Bundesamt. (2019). Lebenserwartung von Männern und Frauen bei der Geburt in Deutschland im Zeitraum der Jahre 1871 bis 2018. Von Statista. https://de.statista. com/statistik/daten/studie/185394/umfrage/entwicklung-der-lebenserwartung-nach-ges chlecht/. Zugegriffen: 05. Nov. 2019.

Staufer, A. (2019). Recht: Telemedizin: Neue Methode mit großem Potenzial. Deutsches Ärzteblatt. https://www.wiso-net.de/document/DAE_3c7cdc3f504b8e52330b6 d5cee5cf51b663023d0. Zugegriffen: 05. März 2020.

Stumpf, S., \& Lorenzen, S. (2017). Der Gartner Hype Cycle. In die richtige Technologie investieren. JAXenter. https://jaxenter.de/gartner-hype-cycle-61770. Zugegriffen: 17. Juni 2020.

Tanaka, F., Isshiki, K., Takahashi, F., Uekusa, M., Sei, R., \& Hayashi, K. (2015). Pepper learns together with children: Development of an educational application. In 2015 IEEE-RAS 15th International Conference on Humanoid Robots (Humanoids) (S. 270-275). IEEE.

Thomée, S., Härenstam, A., \& Hagberg, M. (2011). Mobile phone use and stress, sleep disturbances, and symptoms of depression among young adults - a prospective cohort study. BMC Public Health, 11(1), 1-11. 
Trager, C. S. (2019). Microchip implants: Big brother in a chip coming our way? https://sym antec-blogs.broadcom.com/blogs/featurestories/microchip-implants-big-brother-chipcoming-our-way. Zugegriffen: 10. März 2020.

Tretbar, C. (2017). Der digitale Mensch: Datenchips im Körper - Horror oder Hoffnung? - Politik - Tagesspiegel. https://www.tagesspiegel.de/politik/der-digitale-mensch-datenc hips-im-koerper-horroroder-hoffnung/19840472.html. Zugegriffen: 10. März 2020.

Troianovski, A., \& Grundberg, S. (2012). Nokia's bad call on smartphones. The Wall Street Journal. https://www.wsj.com/articles/SB1000142405270230438800457753100259131 5494. Zugegriffen: 17. Juni 2020.

Truong, A. L., Banu, S., Cao, J., Shah, A., Moukaddam, N., \& Sabharwal, A. (2017, October). Smartphone and online usage-based evaluation in teens (SOLVD-TEEN): Can an app help teens and their parents with depression? In 64th Annual Meeting. AACAP.

Tutt, C. (2020). Spahn bei sensiblen Daten unwissend. Wirtschaftswoche. https://www.wisonet.de/document/WWON_cb36d851dd21c9ac542b7e4372c3459a372d4d99. Zugegriffen: 05. März 2020.

van Lente, H., Spitters, C., \& Peine, A. (2013). Comparing technological hype cycles: Towards a theory. Technological Forecasting and Social Change, 80(8), 1615-1628. https://doi.org/ 10.1016/j.techfore.2012.1.

Verbraucherzentrale NRW e. V. (2019). Gesundheits-Apps: Ab 2020 kommen medizinische Anwendungen auf Rezept. https:/www.verbraucherzentrale.de/wissen/gesundheitpflege/aerzte-und-kliniken/gesundheitsapps-ab-2020-kommen-medizinische-anwend ungen-auf-rezept-41241. Zugegriffen: 05. März 2020.

Verghese, J., Robbins, M., Holtzer, R., Zimmerman, M., Wang, C., Xue, X., \& Lipton, R. B. (2008). Gait dysfunction in mild cognitive impairment syndromes. Journal of the American Geriatrics Society, 56(7), 1244-1251.

Vimarlund, V., \& Wass, S. (2014). Big data, smart homes and ambient assisted living. Yearbook of Medical Informatics, 9(1), 143.

Withings. (2020). Häufig gestellte Fragen zu Move ECG. https://support.withings.com/hc/de/ articles/360014105854-H\%C3\%A4ufig-gestellteFragen-zu-Move-ECG. Zugegriffen: 10. März 2020.

Wu, Y.-H. (2012). Designing robots for the elderly: Appearance issue and beyond. Archives of Gerontology and Geriatrics, 54(1), 121-126.

Zander-Jentsch, B., Wagner, F., Rzayeva, N., \& Busse, R. (2019). Germany. In A. M. Rafferty (Eds.) et. al., Strengthening health systems through nursing: Evidence from 14 European countries. European Observatory on Health Systems and Policies.

Zhang, S., Nugent, C., Lundström, J., \& Sheng, M. (2018). Ambient assisted living for iImprovement of health and quality of life-A special issue of the journal of informatics. In Informatics (Bd. 5, No. 1, S. 4). Multidisciplinary Digital Publishing Institute.

Zugck, C., Nelles, M., Frankenstein, L., Schultz, C., Helms, T., Korb, H., \& Remppis, A. (2005). Telemedizinisches Monitoring bei herzinsuffizienten Patienten. Herzschrittmachertherapie \& Elektrophysiologie, 16(3), 176-182.

Zühlke, K. (2019). Gesundheitsdaten: Sind Chip-Implantate für Menschen akzeptabel? https://www.elektroniknet.de/markt-technik/industrie-40-iot/sindchip-implantatefuer-menschen-akzeptabel-162770.html. Zugegriffen: 12. März 2020. 


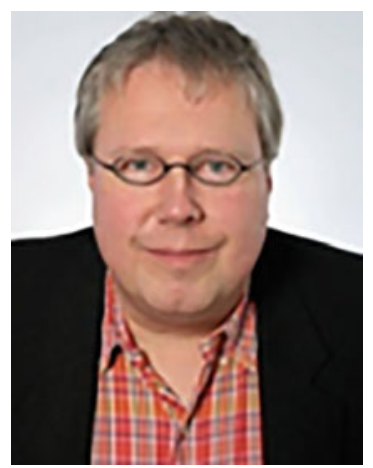

Till Hänisch Seit 2002 Professor für Wirtschaftsinformatik an der DHBW Heidenheim. Schwerpunkt in Forschung und Lehre ist das Internet der Dinge, vor allem in der industriellen Anwendung, insbesondere in der Papierindustrie. Ein zweiter Schwerpunkt sind Sicherheit und Privacy in diesem Kontext.

Open Access Dieses Kapitel wird unter der Creative Commons Namensnennung 4.0 International Lizenz (http://creativecommons.org/licenses/by/4.0/deed.de) veröffentlicht, welche die Nutzung, Vervielfältigung, Bearbeitung, Verbreitung und Wiedergabe in jeglichem Medium und Format erlaubt, sofern Sie den/die ursprünglichen Autor(en) und die Quelle ordnungsgemäß nennen, einen Link zur Creative Commons Lizenz beifügen und angeben, ob Änderungen vorgenommen wurden.

Die in diesem Kapitel enthaltenen Bilder und sonstiges Drittmaterial unterliegen ebenfalls der genannten Creative Commons Lizenz, sofern sich aus der Abbildungslegende nichts anderes ergibt. Sofern das betreffende Material nicht unter der genannten Creative Commons Lizenz steht und die betreffende Handlung nicht nach gesetzlichen Vorschriften erlaubt ist, ist für die oben aufgeführten Weiterverwendungen des Materials die Einwilligung des jeweiligen Rechteinhabers einzuholen.

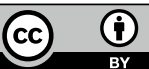

